\title{
Synthesis of Rhamnolipid Derivatives Containing Ester Isosteres
}

Lei Wang and Todd L. Lowary*

Department of Chemistry, University of Alberta, Edmonton, AB T6G 2G2, Canada

Email: tlowary@ualberta.ca

\section{Supporting information}

\section{Table of Contents}

Scheme S1. Synthesis of rhamnosyl trichloroacetimidate donor $5 \quad$ S2

Scheme S2. Determination of enantiomeric excess of compound $13 \quad$ S2

Figure S1. HPLC trace of ethyl (R)-3-((4-methylphenyl)sulfonamido)nonanoate S2

Figure S2. HPLC trace of racemic ethyl 3-((4-methylphenyl)sulfonamido)nonanoate S3

$\begin{array}{ll}\text { Experimental procedures and characterization data } & \text { S3 }\end{array}$

$\begin{array}{ll}\text { References } & \text { S36 }\end{array}$

$\begin{array}{ll}\text { Nuclear Magnetic Resonance (NMR) Spectra } & \text { S37 }\end{array}$ 
Scheme S1: Synthesis of rhamnosyl trichloroacetimidate donor 5

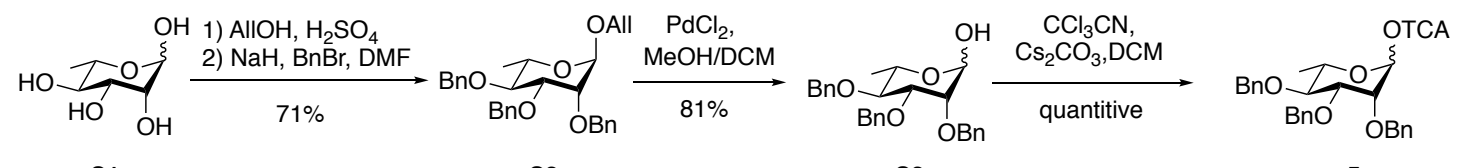

s1

S2

S3

Scheme S2: Determination of enantiomeric excess of compound $\mathbf{1 3}$
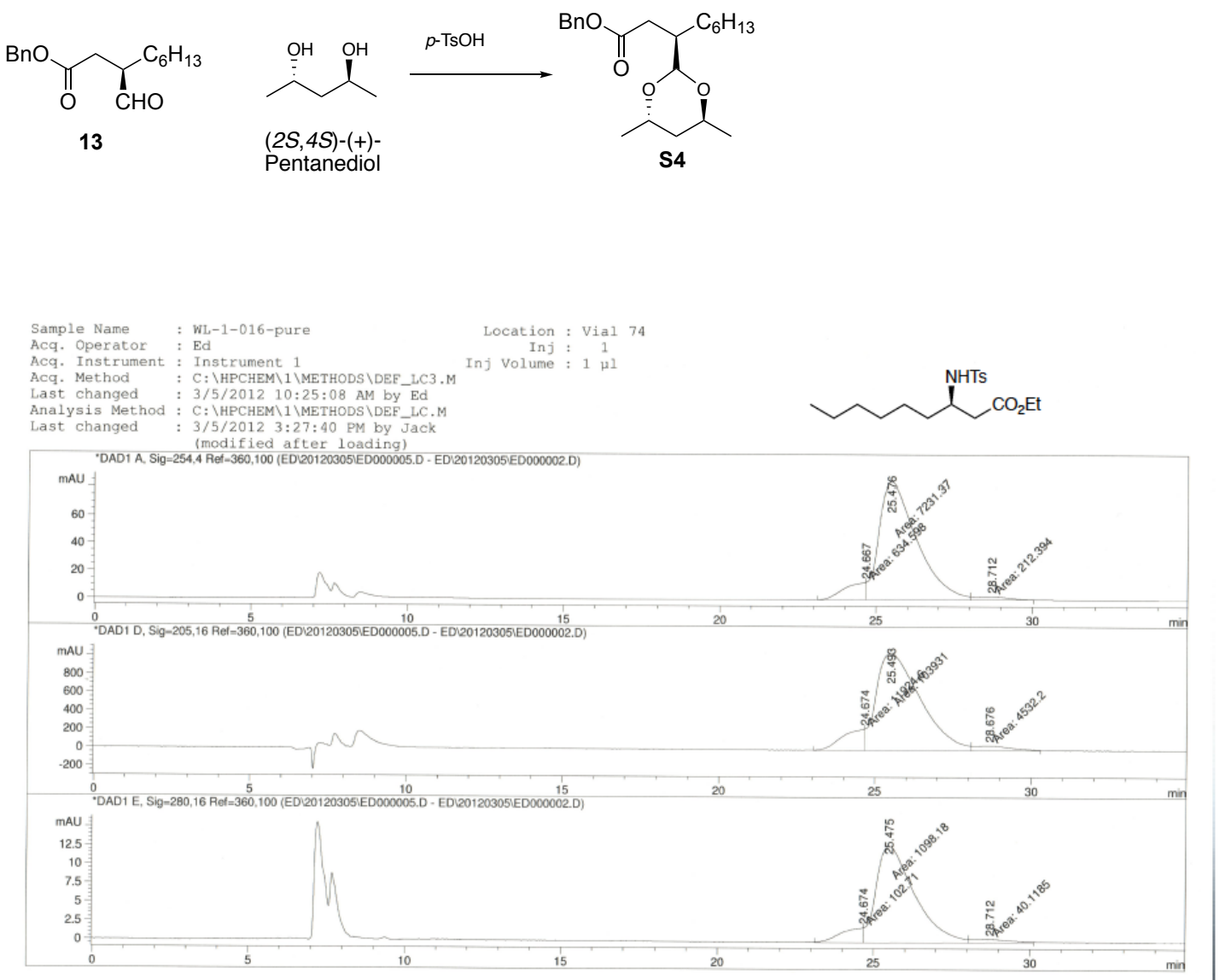

Figure S1: HPLC of ethyl $(R)-3-((4-m e t h y l p h e n y l)$ sulfonamido)nonanoate (N-tosylate of 10). Retention time: $25.47 \mathrm{~min}$. 


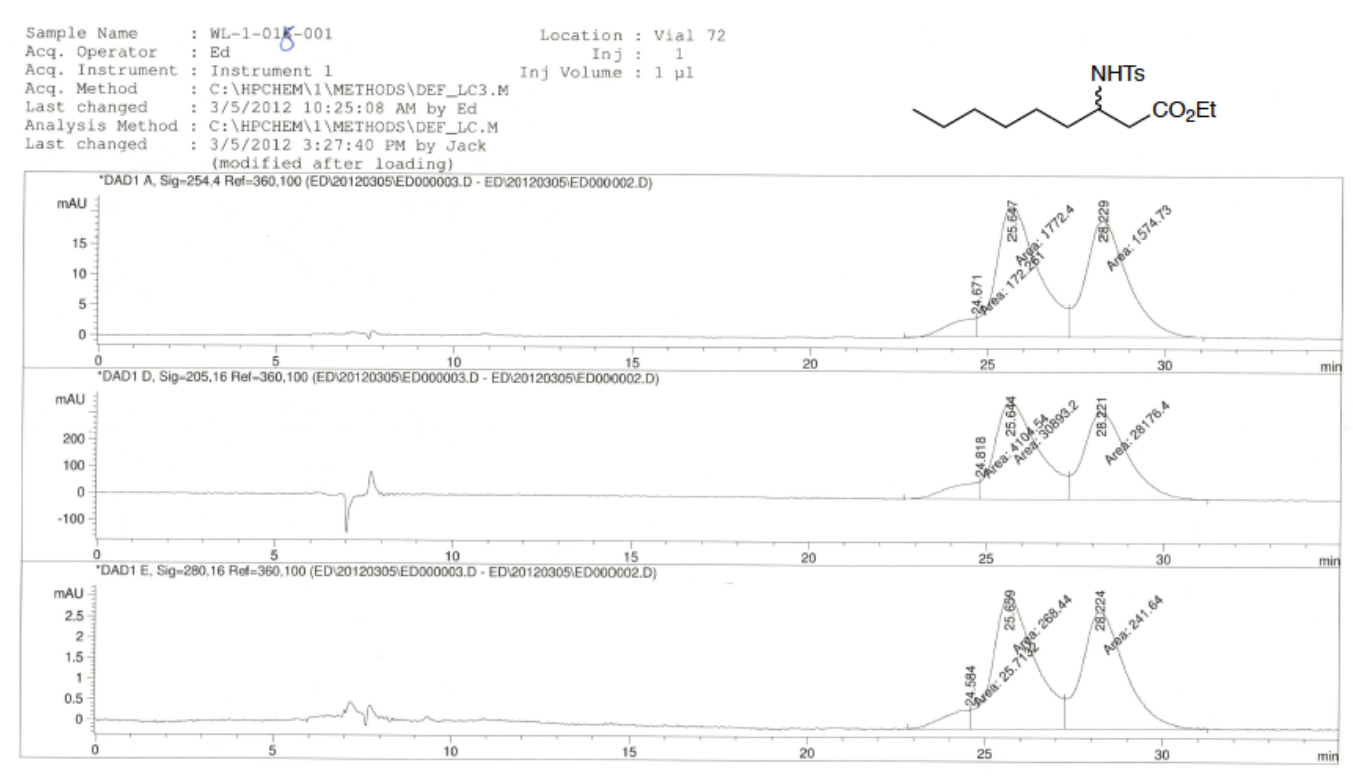

Figure S2: HPLC of racemic ethyl 3-((4-methylphenyl)sulfonamido)nonanoate (Ntosylate of 10). Retention time for $R$-isomer: 25.64 mins; retention time for $S$-isomer: $28.22 \mathrm{mins})$

General Methods: Reactions were carried out in oven-dried glassware. All reagents used were purchased from commercial sources and were used without further purification unless noted. Solvents used in reactions were purified by successive passage through columns of alumina and copper under nitrogen. Unless stated otherwise, all reactions were carried out at r.t. under a positive pressure of argon and were monitored by TLC on silica gel $60 \mathrm{~F}_{254}(0.25 \mathrm{~mm}$, E. Merck). Spots were detected under UV light or by charring with cerium molybdate stain or potassium permanganate stain. Unless otherwise indicated, all column chromatography was performed on silica gel $60(40-60 \mu \mathrm{M})$. The ratio between silica gel and crude product ranged from 100 to $50: 1(\mathrm{w} / \mathrm{w})$. Iatrobeads refers to a beaded silica gel $6 \mathrm{RS}-8060$, which is manufactured by Iatron Laboratories (Tokyo). Optical rotations were measured at $22 \pm 2{ }^{\circ} \mathrm{C}$ at the 
sodium D line $(589 \mathrm{~nm})$ and are in units of $\mathrm{deg} \cdot \mathrm{mL}(\mathrm{dm} \cdot \mathrm{g})^{-1} \cdot{ }^{1} \mathrm{H}$ NMR spectra were recorded at 400, 500 or $700 \mathrm{MHz}$, and chemical shifts are referenced to either TMS $(0.0$ ppm, $\mathrm{CDCl}_{3}$ ) or $\mathrm{HOD}\left(4.78 \mathrm{ppm}, \mathrm{CD}_{3} \mathrm{OD}\right) .{ }^{13} \mathrm{C}$ NMR spectra were recorded at 100 , 125 , or $150 \mathrm{MHz}$, and ${ }^{13} \mathrm{C}$ chemical shifts are referenced to internal $\mathrm{CDCl}_{3}(77.23 \mathrm{ppm}$, $\mathrm{CDCl}_{3}$ ), external dioxane $\mathrm{CD}_{3} \mathrm{OD}$ (48.9 ppm, $\mathrm{CD}_{3} \mathrm{OD}$ ). In the processing of reaction mixtures, solutions of organic solvents were washed with equal amounts of aqueous solutions. Organic solutions were concentrated under vacuum at $<40^{\circ} \mathrm{C}$ (bath). Electrospray mass spectra (time-of-flight analyzer) were recorded on samples suspended in mixtures of THF with $\mathrm{CH}_{3} \mathrm{OH}$ and added $\mathrm{NaCl}$. Electron impact ionization mass spectra were recorded on samples dissolved in $\mathrm{CH}_{2} \mathrm{Cl}_{2}$.

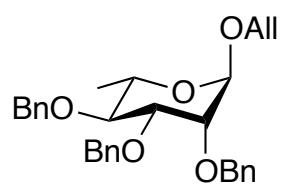

Allyl 2,3,4-tri-O-Benzyl-a-L-rhamnopyranoside (S2): A solution of L-rhamnose monohydrate S1 (5.0 g, $30.5 \mathrm{mmol})$ in dried allyl alcohol $(50 \mathrm{~mL})$ and concentrated $\mathrm{H}_{2} \mathrm{SO}_{4}(0.4 \mathrm{~mL})$ was stirred at $100{ }^{\circ} \mathrm{C}$ for $1 \mathrm{~h}$. The mixture was cooled to r.t. and then, $\mathrm{K}_{2} \mathrm{CO}_{3}(0.4 \mathrm{~g})$ was added to neutralize the solution. After removing allyl alcohol in vacuo, the resulting syrup was partially purified by chromatography (gradient $10 \rightarrow 40 \%$ $\mathrm{CH}_{2} \mathrm{Cl}_{2}$ in $\mathrm{CH}_{3} \mathrm{OH}$ ) to afford crude allyl $\alpha$-L-rhamnopyranoside. This crude residue was taken up in DMF $(200 \mathrm{~mL})$ and treated with $\mathrm{BnBr}(26 \mathrm{~g}, 152 \mathrm{mmol})$. The reaction mixture was cooled $\left(-30^{\circ} \mathrm{C}\right)$ and treated with $\mathrm{NaH}(6.7 \mathrm{~g}$ as a $60 \%$ dispersion in mineral oil, $167 \mathrm{mmol}$ ) and then warmed to r.t. and stirred for $12 \mathrm{~h}$. The solution was cooled to 
$0{ }^{\circ} \mathrm{C}$, the reaction quenched by the addition of $\mathrm{CH}_{3} \mathrm{OH}(50 \mathrm{~mL})$ and the mixture was concentrated. The residue was taken up in $\mathrm{CH}_{2} \mathrm{Cl}_{2}$ washed with brine, dried with $\mathrm{Na}_{2} \mathrm{SO}_{4}$, filtered and concentrated. The resulting residue was purified by chromatography (gradient $5 \rightarrow 7 \%$ EtOAc in hexane) to afford $\mathbf{S 2}(10.3 \mathrm{~g}, 71 \%$ yield) as a foam. $R_{\mathrm{f}} 0.45\left(7: 1\right.$ hexane-EtOAc); $[\alpha]_{\mathrm{D}}=-14.5\left(c 0.3, \mathrm{CH}_{2} \mathrm{Cl}_{2}\right) ;{ }^{1} \mathrm{H}$ NMR $(400$ $\left.\mathrm{MHz}, \mathrm{CDCl}_{3}, \delta_{\mathrm{H}}\right) 7.40-7.27(\mathrm{~m}, 15 \mathrm{H}), 5.84(\mathrm{dddd}, 1 \mathrm{H}, J=17.0,10.5,6.0,5.0 \mathrm{~Hz})$, $5.22(\operatorname{app~dq}, 1 \mathrm{H}, J=17.0,1.5 \mathrm{~Hz}), 5.16(\operatorname{app} \mathrm{dq}, 1 \mathrm{H}, J=10.5,1.6 \mathrm{~Hz}), 4.96(\mathrm{~d}, 1 \mathrm{H}$, $J=10.8 \mathrm{~Hz}), 4.81(\mathrm{~d}, 1 \mathrm{H}, J=1.6 \mathrm{~Hz}), 4.78(\mathrm{~d}, 1 \mathrm{H}, J=12.2 \mathrm{~Hz}), 4.73(\mathrm{~d}, 1 \mathrm{H}, J=$ 12.2 Hz), 4.66 (d, $1 \mathrm{H}, J=10.8 \mathrm{~Hz}), 4.64$ (s, $2 \mathrm{H}), 4.13$ (app ddt, $1 \mathrm{H}, J=13.0,5.0,1.5$ Hz), 3.92 (app ddt, $1 \mathrm{H}, J=13.0,6.0,1.5 \mathrm{~Hz}), 3.90(\mathrm{dd}, 1 \mathrm{H}, J=9.5,3.0 \mathrm{~Hz}), 3.82$ (dd, $1 \mathrm{H}, J=3.0,2.0 \mathrm{~Hz}), 3.73(\mathrm{dq}, 1 \mathrm{H}, J=9.5,6.2 \mathrm{~Hz}), 3.64($ app t, $1 \mathrm{H}, J=9.5 \mathrm{~Hz}), 1.35$ $(\mathrm{d}, J=6.2 \mathrm{~Hz}) ;{ }^{13} \mathrm{C} \mathrm{NMR}\left(100 \mathrm{MHz}, \mathrm{CDCl}_{3}, \delta_{\mathrm{C}}\right) 140.62,140.61,140.4,135.8,130.3$, $130.0,129.9,129.6,129.5,119.1,99.2,82.5,82.2,77.4,76.9,74.8,74.2,70.1,69.7$, 20.0; HRMS (ESI) m/z $[\mathrm{M}+\mathrm{Na}]^{+}$Calcd for $\mathrm{C}_{30} \mathrm{H}_{34} \mathrm{NaO}_{5}$ 497.2304; Found 497.2310. The ${ }^{1} \mathrm{H}(400 \mathrm{MHz})$ and ${ }^{13} \mathrm{C} \mathrm{NMR}(100 \mathrm{MHz})$ data of this material were in good agreement with those reported. ${ }^{1}$<smiles>N=C(OC1OC2C(Br)OC(OC(=O)C2Br)C1OCc1ccccc1)C(Cl)(Cl)Cl</smiles>

2,3,4-tri- $O$-Benzyl- $\alpha$-L-rhamnopyranosyl trichloroacetimidate (5): To a solution of S2 (1.2 g, $2.53 \mathrm{mmol})$ in $\mathrm{CH}_{3} \mathrm{OH}(20 \mathrm{~mL})$ and $\mathrm{CH}_{2} \mathrm{Cl}_{2}(20 \mathrm{~mL})$ at r.t. was added $\mathrm{PdCl}_{2}$ (106 mg, $0.6 \mathrm{mmol}$ ). The mixture was stirred at r.t. for $16 \mathrm{~h}$. After completion of the 
reaction, the mixture was filtered through a plug of Celite and the filtrate was concentrated and the resulting residue was purified by chromatography $(25 \%$ EtOAc in hexane) to give $\mathbf{S 3}(0.89 \mathrm{~g}, 81 \%$ yield) as a white solid. A slurry of $\mathbf{S 3}$ (100 mg, 0.23 mmol), $\mathrm{Cl}_{3} \mathrm{CCN}(0.33 \mathrm{~g}, 2.3 \mathrm{mmol})$, and $\mathrm{Cs}_{2} \mathrm{CO}_{3}(0.37 \mathrm{~g}, 1.15 \mathrm{mmol})$ in $\mathrm{CH}_{2} \mathrm{Cl}_{2}(10$ $\mathrm{mL}$ ) was stirred for $3.5 \mathrm{~h}$. The reaction mixture was filtered through a short pad of Celite and the pad was washed with dry $\mathrm{CH}_{2} \mathrm{Cl}_{2}$. The filtrate was concentrated to give $0.13 \mathrm{~g}$ of a slightly yellowish oil $\mathbf{5}$, which was immediately used in the glycosylation step without further purification.<smiles>COC(=O)CC(O)c1ccccc1</smiles>

Methyl 3-(R)-hydroxy-nonanoate (15): To a solution of methyl-3-oxo-nonanoate $\mathbf{1 4}^{2}$ $(1.0 \mathrm{~g}, 5.4 \mathrm{mmol})$ in dry $\mathrm{CH}_{3} \mathrm{OH}(25 \mathrm{~mL})$ was added $(R)-\mathrm{BINAP}^{-\mathrm{RuCl}_{2}}(12 \mathrm{mg})$. The reaction mixture was placed in a hydrogenation autoclave and pressurized with $\mathrm{H}_{2}$ (55 PSI) and brought to $60{ }^{\circ} \mathrm{C}$ for $12 \mathrm{~h}$. After cooling, the reaction mixture was diluted with brine, extracted with $\mathrm{CH}_{2} \mathrm{Cl}_{2}$ and subsequently washed with water and brine. The organic phase was dried over $\mathrm{Na}_{2} \mathrm{SO}_{4}$, filtered and concentrated. The resulting residue was purified by chromatography (gradient $16 \rightarrow 20 \%$ EtOAc in hexane) to afford $\mathbf{1 5}$ ( $0.89 \mathrm{~g}, 88 \%$ yield, $93 \%$ ee) as a colorless oil. The enantioselectivity was determined by coupling 15 with $(S)-(+)-O$-acetylmandelic acid. Then, the $\mathrm{OCH}_{3}$ signals in ${ }^{1} \mathrm{H}$ NMR spectra of the resulting mixture were integrated to determine the ratio between the $(R)$ $\beta$-hydroxy-ester (3.64 ppm) and $(S)$ - $\beta$-hydroxy-ester (3.37 ppm). Data for 15: $R_{\mathrm{f}} 0.47$ (3:1 hexane-EtOAc); $[\alpha]_{\mathrm{D}}=-17.0\left(c 0.4, \mathrm{CH}_{2} \mathrm{Cl}_{2}\right) ;{ }^{1} \mathrm{H}$ NMR $\left(400 \mathrm{MHz}, \mathrm{CDCl}_{3}, \delta_{\mathrm{H}}\right)$ 
4.04-3.97 (m, $1 \mathrm{H}), 3.72(\mathrm{~s}, 3 \mathrm{H}), 2.84$ (d, $1 \mathrm{H}, J=4.2 \mathrm{~Hz}), 2.52(\mathrm{dd}, 1 \mathrm{H}, J=16.4,3.2$

Hz), $2.41(\mathrm{dd}, 1 \mathrm{H}, J=16.4,9.0 \mathrm{~Hz}), 1.56-1.27(\mathrm{~m}, 10 \mathrm{H}), 0.88(\mathrm{t}, 3 \mathrm{H}, J=7.2 \mathrm{~Hz})$;

${ }^{13} \mathrm{C}$ NMR $\left(100 \mathrm{MHz}, \mathrm{CDCl}_{3}, \delta_{\mathrm{C}}\right)$ 173.1, 67.6, 51.3, 40.7, 36.1, 31.3, 28.8, 25.0, 22.2,

13.6; HRMS (ESI) m/z [M + Na] ${ }^{+}$Calcd for $\mathrm{C}_{10} \mathrm{H}_{20} \mathrm{NaO}_{3}$ 211.1310; Found 211.1307.<smiles>COC(=O)CC([18OH])[18OH]</smiles>

Methyl (R)-3-tert-butyldimethylsilyloxy-nonanoate (11): A solution of $\beta$-hydroxy ester 15 (0.77 g, $4.1 \mathrm{mmol})$ in pyridine-THF $(2: 1,30 \mathrm{~mL})$ was treated with $\mathrm{AgNO}_{3}$ $(0.77 \mathrm{~g}, 4.5 \mathrm{mmol})$ and $\mathrm{TBSCl}(0.68 \mathrm{~g}, 4.5 \mathrm{mmol})$ and the mixture was stirred at r.t. for $4 \mathrm{~h}$. The mixture was filtered and concentrated. The residue was dissolved in $\mathrm{CH}_{2} \mathrm{Cl}_{2}$ ( $50 \mathrm{~mL}$ ) followed by washing with $1 \mathrm{M}$ of $\mathrm{HCl}$, saturated aqueous $\mathrm{NaHCO}_{3}$, and brine. The organic phase was dried $\left(\mathrm{Na}_{2} \mathrm{SO}_{4}\right)$, filtered, and concentrated. The residue was purified by column chromatography (gradient $5 \rightarrow 10 \%$ EtOAc in hexane) to afford 11 $(1.1 \mathrm{~g}, 92 \%$ yield $)$ as a colorless oil; $R_{\mathrm{f}} 0.83(3: 1$ hexane-EtOAc $) ;[\alpha]_{\mathrm{D}}=-19.0(c 0.5$, $\left.\mathrm{CH}_{2} \mathrm{Cl}_{2}\right) ;{ }^{1} \mathrm{H}$ NMR $\left(400 \mathrm{MHz}, \mathrm{CDCl}_{3}, \delta_{\mathrm{H}}\right) 4.16-4.09(\mathrm{~m}, 1 \mathrm{H}), 3.67(\mathrm{~s}, 3 \mathrm{H}), 2.46(\mathrm{dd}$, $1 \mathrm{H}, J=14.6,7.0 \mathrm{~Hz}), 2.41(\mathrm{dd}, 1 \mathrm{H}, J=14.6,5.8 \mathrm{~Hz}), 1.51-1.27(\mathrm{~m}, 10 \mathrm{H}), 0.88(\mathrm{t}, 3$ $\mathrm{H}, J=7.2 \mathrm{~Hz}), 0.87(\mathrm{~s}, 9 \mathrm{H}), 0.06(\mathrm{~s}, 3 \mathrm{H}), 0.03(\mathrm{~s}, 3 \mathrm{H}) ;{ }^{13} \mathrm{C} \mathrm{NMR}\left(100 \mathrm{MHz}, \mathrm{CDCl}_{3}\right.$, $\left.\delta_{\mathrm{C}}\right) 171.9,69.1,51.0,42.1,37.2,31.4,28.9,25.4,24.5,22.2,17.6,13.6,-4.9,-5.2$; HRMS (ESI) m/z [M + Na $]^{+}$Calcd for $\mathrm{C}_{16} \mathrm{H}_{34} \mathrm{NaO}_{3} \mathrm{Si}$ : 325.2175; Found: 325.2170 . 
<smiles>CCCCCCC([OH2+])CC(=O)O</smiles>

(R)-3-tert-butyldimethylsilyloxy-nonanoic acid (16): A solution of 11 (0.43 g, 1.4 mmol) in $\mathrm{CH}_{3} \mathrm{OH}$-water $(4: 1,25 \mathrm{~mL})$ was treated with $\mathrm{LiOH} \cdot \mathrm{H}_{2} \mathrm{O}(0.3 \mathrm{~g}, 7.1 \mathrm{mmol})$ and the mixture was stirred at $50{ }^{\circ} \mathrm{C}$ overnight. After cooling to r.t., the reaction mixture was acidified to $\mathrm{pH} 5$ by the addition of $1 \mathrm{M} \mathrm{HCl}$, diluted with water and extracted with $\mathrm{CH}_{2} \mathrm{Cl}_{2}$. The organic phase was washed with brine, dried over $\mathrm{Na}_{2} \mathrm{SO}_{4}$, filtered, and concentrated. The resulting residue was purified by chromatography (gradient $5 \rightarrow 10 \%$ EtOAc in hexane) to afford $16(0.37 \mathrm{~g}, 90 \%$ yield $)$ as a colorless oil. $R_{\mathrm{f}} 0.44(3: 1$ hexane-EtOAc); $[\alpha]_{\mathrm{D}}=+3.5\left(c \quad 0.4, \mathrm{CH}_{2} \mathrm{Cl}_{2}\right) ;{ }^{1} \mathrm{H}$ NMR $\left(400 \mathrm{MHz}, \mathrm{CDCl}_{3}, \delta_{\mathrm{H}}\right)$ 4.13-4.07 (m, $1 \mathrm{H}), 2.55(\mathrm{dd}, 1 \mathrm{H}, J=15.0,5.2 \mathrm{~Hz}), 2.49(\mathrm{dd}, 1 \mathrm{H}, J=15.0,5.8 \mathrm{~Hz})$, 1.57-1.27 (m, $10 \mathrm{H}), 0.90$ (s, $9 \mathrm{H}), 0.89$ (t, $3 \mathrm{H}, J=7.2 \mathrm{~Hz}), 0.11$ (s, $3 \mathrm{H}), 0.09$ (s, 3 $\mathrm{H}) ;{ }^{13} \mathrm{C} \mathrm{NMR}\left(100 \mathrm{MHz}, \mathrm{CDCl}_{3}, \delta_{\mathrm{C}}\right)$ 177.2, 69.1, 41.2, 36.7, 31.3, 28.8, 25.3, 24.8, 22.2, 17.6, 13.6, -4.9, -5.3; HRMS (ESI) $\mathrm{m} / \mathrm{z}[\mathrm{M}+\mathrm{Na}]^{+}$Calcd for $\mathrm{C}_{15} \mathrm{H}_{32} \mathrm{NaO}_{3} \mathrm{Si}$ 311.2018; Found 311.2025.<smiles>C/C=C/C(=O)OCC</smiles>

(E)-ethyl 2-nonenoate (18): To a solution of triethylphosphonoacetate 17 (16.0 g, 71 $\mathrm{mmol})$ in THF $(90 \mathrm{~mL})$ at $-78^{\circ} \mathrm{C}$ was added $1.6 \mathrm{M} n$-BuLi in hexane (44 mL, $\left.71 \mathrm{mmol}\right)$. The reaction mixture was stirred for $30 \mathrm{~min}$ and a solution of heptanaldehyde $(6.2 \mathrm{~g}, 54$ mmol) in THF $(90 \mathrm{~mL})$ was added dropwise at $-78{ }^{\circ} \mathrm{C}$. The resulting mixture was stirred at $-78{ }^{\circ} \mathrm{C}$ for $1.5 \mathrm{~h}$ and then saturated $\mathrm{NH}_{4} \mathrm{Cl}$ solution was added to quench the 
reaction. After the reaction mixture was warmed to r.t., the product was extracted with ether and the organic layer was washed with a satd aq solution of $\mathrm{NaHCO}_{3}$, brine, dried over $\mathrm{Na}_{2} \mathrm{SO}_{4}$, filtered and concentrated. The resulting residue was purified by chromatography (gradient $0.3 \rightarrow 0.5 \%$ EtOAc in hexane) to afford $\mathbf{1 8}(10.8 \mathrm{~g}, 82 \%$ yield) as a colorless oil. $R_{\mathrm{f}} 0.20$ (hexane); ${ }^{1} \mathrm{H}$ NMR $\left(400 \mathrm{MHz}, \mathrm{CDCl}_{3}, \delta_{\mathrm{H}}\right) 6.97(\mathrm{dt}, 1 \mathrm{H}, J=$ 15.5, 7.0 Hz), $5.81(\mathrm{dt}, 1 \mathrm{H}, J=15.5,1.5 \mathrm{~Hz}), 4.18(\mathrm{q}, 2 \mathrm{H}, J=7.2 \mathrm{~Hz}), 2.22-2.16(\mathrm{~m}$, $2 \mathrm{H}), 1.49-1.23(\mathrm{~m}, 11 \mathrm{H}), 0.89(\mathrm{t}, 3 \mathrm{H}, J=7.2 \mathrm{~Hz}) ;{ }^{13} \mathrm{C} \mathrm{NMR}\left(100 \mathrm{MHz}, \mathrm{CDCl}_{3}, \delta_{\mathrm{C}}\right)$ 166.4, 149.1, 120.8, 59.7, 31.8, 31.2, 28.4, 27.6, 22.1, 13.9, 13.6; HRMS (ESI) m/z [M $+\mathrm{H}]^{+}$Calcd for $\mathrm{C}_{11} \mathrm{H}_{21} \mathrm{O}_{2}$ 185.1542; Found 185.1551. The ${ }^{1} \mathrm{H}(400 \mathrm{MHz})$ and ${ }^{13} \mathrm{C} \mathrm{NMR}$ $(100 \mathrm{MHz})$ data of this material were in good agreement with those reported. ${ }^{3}$<smiles>CCOC(=O)CC(Cc1ccccc1)N(Cc1ccccc1)C(C)c1ccccc1</smiles>

(R)-ethyl 3-(benzyl((R)-1-phenylethyl)amino)nonanoate (19) To a solution of $(R)$ $N$-benzyl-1-phenylethanamine $(0.41 \mathrm{~g}, 1.9 \mathrm{mmol})$ in THF $(12 \mathrm{~mL})$ was added $n$-BuLi (2.5 $\mathrm{M}$ in hexane, $0.73 \mathrm{~mL}, 1.8 \mathrm{mmol}$ ) at $-78^{\circ} \mathrm{C}$ and the reaction mixture was stirred for $30 \mathrm{~min}$. A solution of $\mathbf{1 8}(0.30 \mathrm{~g}, 1.2 \mathrm{mmol})$ in THF $(4.0 \mathrm{~mL})$ was added dropwise at $-78^{\circ} \mathrm{C}$ and the resulting mixture was stirred for $2.5 \mathrm{~h}$. A solution of 4-bromo-2,6-ditert-butylphenol (1.0 g, $3.6 \mathrm{mmol})$ in THF (4 mL) was added, the mixture was warmed to r.t. and then the solvent was evaporated. The crude material was washed with brine and extracted with $\mathrm{CH}_{2} \mathrm{Cl}_{2}$. The organic phase was dried over $\mathrm{Na}_{2} \mathrm{SO}_{4}$, filtered and concentrated. The resulting residue was purified by chromatography (gradient $0 \rightarrow 0.3 \%$ 
EtOAc in hexane $)$ to afford $19(0.55 \mathrm{~g}, 86 \%$ yield $)$ as a colorless oil. $[\alpha]_{\mathrm{D}}=+7.0(c 0.3$, $\left.\mathrm{CH}_{2} \mathrm{Cl}_{2}\right) ;{ }^{1} \mathrm{H} \mathrm{NMR}\left(400 \mathrm{MHz}, \mathrm{CDCl}_{3}, \delta_{\mathrm{H}}\right) 7.44-7.42(\mathrm{~m}, 2 \mathrm{H}), 7.36-7.20$ (m, $\left.8 \mathrm{H}\right), 4.05$ (dq, $1 \mathrm{H}, J=10.8,7.2 \mathrm{~Hz}), 3.99(\mathrm{dq}, 1 \mathrm{H}, J=10.8,7.2 \mathrm{~Hz}), 3.84(\mathrm{q}, 1 \mathrm{H}, J=7.0 \mathrm{~Hz})$, $3.80(\mathrm{~d}, 1 \mathrm{H}, J=14.8 \mathrm{~Hz}), 3.55(\mathrm{~d}, 1 \mathrm{H}, J=14.8 \mathrm{~Hz}), 3.34-3.27(\mathrm{~m}, 1 \mathrm{H}), 2.06(\mathrm{dd}, 1$ $\mathrm{H}, J=14.4,4.8 \mathrm{~Hz}), 2.00(\mathrm{dd}, 1 \mathrm{H}, J=14.4,8.0 \mathrm{~Hz}), 1.56-1.47$ (m, $2 \mathrm{H}), 1.34(\mathrm{~d}, 3 \mathrm{H}$, $J=7.0 \mathrm{~Hz}), 1.32-1.22(\mathrm{~m}, 8 \mathrm{H}), 1.19(\mathrm{t}, 3 \mathrm{H}, J=7.2 \mathrm{~Hz}), 0.89(\mathrm{t}, 3 \mathrm{H}, J=7.2 \mathrm{~Hz}) ;{ }^{13} \mathrm{C}$ NMR (100 MHz, $\left.\mathrm{CDCl}_{3}, \delta_{\mathrm{C}}\right)$ 172.5, 142.9, 141.4, 127.9, 127.8, 127.6, 127.5, 126.4, 126.2, 59.7, 57.6, 53.7, 49.6, 36.5, 33.1, 31.5, 28.9, 26.6, 22.3, 19.3, 13.8, 13.7; HRMS (ESI) $\mathrm{m} / \mathrm{z}[\mathrm{M}+\mathrm{H}]^{+}$Calcd for $\mathrm{C}_{26} \mathrm{H}_{38} \mathrm{NO}_{2}$ 396.2897; Found 396.2889.<smiles>CCOC(=O)CC(N)c1ccccc1</smiles>

(R)-ethyl 3-aminononanoate (10): To a solution of 19 (2.0 g, $5.0 \mathrm{mmol})$ in ethanol (30 $\mathrm{mL})$ was added $10 \%$ palladium on charcoal $(0.10 \mathrm{~g})$ and the mixture was hydrogenolysed (hydrogen pressure: $60 \mathrm{psi}$ ) at r.t. for $16 \mathrm{~h}$. The mixture was filtered and the solution was concentrated to afford the target compound $\mathbf{1 0}$ ( $0.94 \mathrm{~g}, 92 \%$ yield). $R_{\mathrm{f}} 0.20\left(\right.$ EtOAc); $[\alpha]_{\mathrm{D}}=-4.9\left(c 0.7, \mathrm{CH}_{2} \mathrm{Cl}_{2}\right) ;{ }^{1} \mathrm{H} \mathrm{NMR}\left(400 \mathrm{MHz}, \mathrm{CDCl}_{3}, \delta_{\mathrm{H}}\right) 4.15(\mathrm{q}$, $2 \mathrm{H}, J=7.2 \mathrm{~Hz}), 3.21-3.15(\mathrm{~m}, 1 \mathrm{H}), 2.46(\mathrm{dd}, 1 \mathrm{H}, J=15.6,4.0 \mathrm{~Hz}), 2.25(\mathrm{dd}, 1 \mathrm{H}, J$ $=15.6,8.8 \mathrm{~Hz}), 1.38-1.25(\mathrm{~m}, 11 \mathrm{H}), 0.89(\mathrm{t}, 3 \mathrm{H}, J=7.2 \mathrm{~Hz}) ;{ }^{13} \mathrm{C} \mathrm{NMR}(100 \mathrm{MHz}$, $\left.\mathrm{CDCl}_{3}, \delta_{\mathrm{C}}\right) 172.6,60.5,48.5,41.4,36.8,31.7,29.2,25.9,22.6,14.2,12.0 ; \mathrm{HRMS}(\mathrm{ESI})$ $\mathrm{m} / \mathrm{z}[\mathrm{M}+\mathrm{H}]^{+}$Calcd for $\mathrm{C}_{11} \mathrm{H}_{24} \mathrm{NO}_{2}$ 202.1802; Found 202.1801. 
<smiles>CCCCCCC(CCCC)CC(=O)NC(CCCCC)CC(=O)OCC</smiles>

(R)-ethyl 3-((R)-3-((tert-butyldimethylsilyl)oxy)nonanamido)nonanoate (20): To a solution of $10(0.11 \mathrm{~g}, 0.54 \mathrm{mmol})$ in DMF $(5.0 \mathrm{~mL})$ was added $16(0.15 \mathrm{~g}, 0.52 \mathrm{mmol})$, $\operatorname{HBTU}(0.25 \mathrm{~g}, 0.78 \mathrm{mmol})$ and DIEA $(0.10 \mathrm{~g}, 0.78 \mathrm{mmol})$. The mixture was stirred at r.t. overnight and then water was added and the mixture was extracted with EtOAc. The organic phase was washed with brine, dried over $\mathrm{Na}_{2} \mathrm{SO}_{4}$, filtered and concentrated. The resulting residue was purified by chromatography (silica gel, gradient $10 \rightarrow 12.5 \%$ EtOAc in hexane) to afford $20(0.16 \mathrm{~g}, 66 \%$ yield $)$ as a colorless oil. $R_{\mathrm{f}} 0.42$ (6:1 hexane-EtOAc); $[\alpha]_{\mathrm{D}}=+16.3\left(\right.$ c $\left.0.3, \mathrm{CH}_{2} \mathrm{Cl}_{2}\right) ;{ }^{1} \mathrm{H} \mathrm{NMR}\left(400 \mathrm{MHz}, \mathrm{CDCl}_{3}, \delta_{\mathrm{H}}\right) 6.60(\mathrm{~d}$, $1 \mathrm{H}, J=8.4 \mathrm{~Hz}), 4.29-4.22(\mathrm{~m}, 1 \mathrm{H}), 4.16-4.09(\mathrm{~m}, 2 \mathrm{H}), 4.02$ (app tt, $1 \mathrm{H}, J=6.2$, $4.8 \mathrm{~Hz}), 2.52(\mathrm{dd}, 1 \mathrm{H}, J=15.6,5.6 \mathrm{~Hz}), 2.46(\mathrm{dd}, 1 \mathrm{H}, J=15.6,6.0 \mathrm{~Hz}), 2.42(\mathrm{dd}, 1$ $\mathrm{H}, J=14.8,4.8 \mathrm{~Hz}), 2.30(\mathrm{dd}, 1 \mathrm{H}, J=14.8,4.8 \mathrm{~Hz}), 1.56-1.48(\mathrm{~m}, 4 \mathrm{H}), 1.37-1.24$ (m, $19 \mathrm{H}), 0.90(\mathrm{~s}, 9 \mathrm{H}), 0.89-0.86(\mathrm{~m}, 6 \mathrm{H}), 0.094$ (s, $3 \mathrm{H}), 0.091$ (s, $3 \mathrm{H}) ;{ }^{13} \mathrm{C}$ NMR $\left(100 \mathrm{MHz}, \mathrm{CDCl}_{3}, \delta_{\mathrm{C}}\right) 171.1,170.0,69.5,60.0,45.5,43.6,38.8,36.1,33.9,31.3,28.9$, 28.6, 25.7, 25.4, 25.3, 22.2, 22.1, 17.6, 13.8, 13.6, -5.1; HRMS (ESI) m/z [M + H] ${ }^{+}$ Calcd for $\mathrm{C}_{26} \mathrm{H}_{54} \mathrm{NO}_{4} \mathrm{Si}$ 472.3817; Found 472.3815.<smiles>CCCCCC(CC(=O)OCC)NC(=O)C[C@H](O)c1ccccc1</smiles>

(R)-ethyl 3-((R)-3-hydroxynonanamido)nonanoate (6): A solution of 20 (0.52 g, 1.1 mmol) in $1.5 \% \mathrm{HCl}$ in $\mathrm{CH}_{3} \mathrm{OH}(40 \mathrm{~mL})$ was stirred for $1 \mathrm{~h}$. The reaction mixture was 
neutralized by the addition of saturated aqueous $\mathrm{NaHCO}_{3}$, diluted with water and extracted with $\mathrm{CH}_{2} \mathrm{Cl}_{2}$. The organic phase was washed with brine, dried over $\mathrm{Na}_{2} \mathrm{SO}_{4}$, filtered and concentrated. The resulting residue was purified by chromatography (gradient $20 \rightarrow 50 \%$ EtOAc in hexane) to afford 6 ( $0.36 \mathrm{~g}, 92 \%$ yield) as a colorless oil. $R_{\mathrm{f}} 0.40\left(1: 1\right.$ hexane-EtOAc); $[\alpha]_{\mathrm{D}}=+4.9\left(c 0.6, \mathrm{CH}_{2} \mathrm{Cl}_{2}\right) ;{ }^{1} \mathrm{H} \mathrm{NMR}\left(400 \mathrm{MHz}, \mathrm{CDCl}_{3}\right.$, $\left.\delta_{\mathrm{H}}\right) 6.22(\mathrm{~d}, 1 \mathrm{H}, J=9.2 \mathrm{~Hz}), 4.29-4.21(\mathrm{~m}, 1 \mathrm{H}), 4.18-4.10(\mathrm{~m}, 2 \mathrm{H}), 3.99-3.93(\mathrm{~m}, 1$ H), $3.76(\mathrm{~d}, 1 \mathrm{H}, J=3.2 \mathrm{~Hz}), 2.56(\mathrm{dd}, 1 \mathrm{H}, J=15.6,4.8 \mathrm{~Hz}), 2.44(\mathrm{dd}, 1 \mathrm{H}, J=15.6$, $6.0 \mathrm{~Hz}), 2.34(\mathrm{dd}, 1 \mathrm{H}, J=14.8,2.8 \mathrm{~Hz}), 2.22(\mathrm{dd}, 1 \mathrm{H}, J=14.8,9.2 \mathrm{~Hz}), 1.55-1.40$ (m, $4 \mathrm{H}), 1.34-1.24(\mathrm{~m}, 19 \mathrm{H}), 0.90-0.86(\mathrm{~m}, 6 \mathrm{H}) ;{ }^{13} \mathrm{C} \mathrm{NMR}\left(100 \mathrm{MHz}, \mathrm{CDCl}_{3}, \delta_{\mathrm{C}}\right)$ $171.7,171.5,68.4,60.4,45.7,42.7,38.2,36.5,33.8,31.4,31.3,28.8,28.6,25.7,25.1$, 22.2, 22.1, 13.76, 13.66, 13.63; HRMS (ESI) m/z $[\mathrm{M}+\mathrm{H}]^{+}$Calcd for $\mathrm{C}_{20} \mathrm{H}_{40} \mathrm{NO}_{4}$ 358.2952; Found 358.2949.

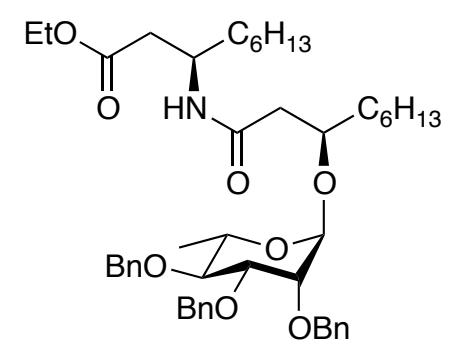

Ethyl $(R)-3-N$-[2,3,4-tri-O-benzyl- $\alpha-L-$-rhamnopyranosyl-(1 $\rightarrow 3)-(R)-3$ '-O-nonana mido]nonanoate (21): A mixture of acceptor $6 \quad(40 \mathrm{mg}, 0.11 \mathrm{mmol})$, trichloroacetimidate donor $5(0.13 \mathrm{~g}, 0.22 \mathrm{mmol})$ and powdered $4 \AA$ molecular sieves was suspended in anhydrous $\mathrm{Et}_{2} \mathrm{O}(10 \mathrm{~mL})$ and stirred at r.t. for $10 \mathrm{~min}$. The solution was then cooled to $0{ }^{\circ} \mathrm{C}$, and then TMSOTf $(10 \mu \mathrm{L})$ was added. The solution was stirred for $1 \mathrm{~h}$ before $\mathrm{Et}_{3} \mathrm{~N}(0.50 \mathrm{~mL})$ was added and the mixture was filtered. The filtrate was 
concentrated and the resulting residue was purified by chromatography (gradient $16 \rightarrow 20 \%$ EtOAc in hexane) to afford 21 (53 mg, 62\% yield) as a colorless oil; $R_{\mathrm{f}} 0.55$ (2:1 hexane-EtOAc); $[\alpha]_{\mathrm{D}}=+2.7\left(c \quad 0.6, \mathrm{CH}_{2} \mathrm{Cl}_{2}\right) ;{ }^{1} \mathrm{H} \mathrm{NMR}\left(400 \mathrm{MHz}, \mathrm{CDCl}_{3}, \delta_{\mathrm{H}}\right)$ 7.38-7.26 (m, $15 \mathrm{H}), 6.39$ (d, $1 \mathrm{H}, J=9.2 \mathrm{~Hz}), 4.92(\mathrm{~d}, 1 \mathrm{H}, J=10.8 \mathrm{~Hz}), 4.84(\mathrm{~d}, 1$ $\mathrm{H}, J=2.0 \mathrm{~Hz}), 4.77(\mathrm{~d}, 1 \mathrm{H}, J=12.8 \mathrm{~Hz}), 4.71(\mathrm{~d}, 1 \mathrm{H}, J=12.8 \mathrm{~Hz}), 4.65-4.63(\mathrm{~m}, 3$ H), 4.24-4.16 (m, $1 \mathrm{H}), 4.12-3.93(\mathrm{~m}, 3 \mathrm{H}), 3.82(\mathrm{dd}, 1 \mathrm{H}, J=9.2,3.2 \mathrm{~Hz}), 3.72(\mathrm{dd}$, $1 \mathrm{H}, J=3.0,2.0 \mathrm{~Hz}), 3.71-3.64(\mathrm{~m}, 1 \mathrm{H}), 3.60(\operatorname{app~t}, 1 \mathrm{H}, J=9.2 \mathrm{~Hz}), 2.53(\mathrm{dd}, 1 \mathrm{H}$, $J=16.0,5.6 \mathrm{~Hz}), 2.43(\mathrm{dd}, 1 \mathrm{H}, J=16.0,4.8 \mathrm{~Hz}), 2.40(\mathrm{dd}, 1 \mathrm{H}, J=14.4,6.0 \mathrm{~Hz})$, $2.32(\mathrm{dd}, 1 \mathrm{H}, J=14.4,5.6 \mathrm{~Hz}), 1.49-1.41(\mathrm{~m}, 4 \mathrm{H}), 1.31(\mathrm{~d}, 3 \mathrm{H}, J=6.4 \mathrm{~Hz}), 1.34-1.21$ (m, $16 \mathrm{H}), 1.17$ (t, $3 \mathrm{H}, J=7.2 \mathrm{~Hz}), 0.90$ (t, $3 \mathrm{H}, J=7.2 \mathrm{~Hz}), 0.87$ (t, $3 \mathrm{H}, J=7.2 \mathrm{~Hz})$; ${ }^{13} \mathrm{C}$ NMR $\left(125 \mathrm{MHz}, \mathrm{CDCl}_{3}, \delta_{\mathrm{C}}\right) 172.1,169.8,138.74,138.69,138.3,128.39,128.36$, $128.29,128.1,127.9,127.5,96.8,80.6,80.2,75.3,75.0,74.4,72.9,72.2,68.5,60.5$, $45.7,42.6,38.2,34.1,33.0,31.7,29.3,29.0,26.2,24.9,22.64,22.57,18.0,14.14,14.11$, 14.06; gHSQC 2-D NMR (without ${ }^{1} \mathrm{H}$ decoupling): ${ }^{1} J_{\mathrm{Cl}, \mathrm{H} 1}=170.2 \mathrm{~Hz}$. HRMS (ESI) $\mathrm{m} / \mathrm{z}[\mathrm{M}+\mathrm{Na}]^{+}$Calcd for $\mathrm{C}_{47} \mathrm{H}_{67} \mathrm{NNaO}_{8}$ 796.4759; Found 796.4749.

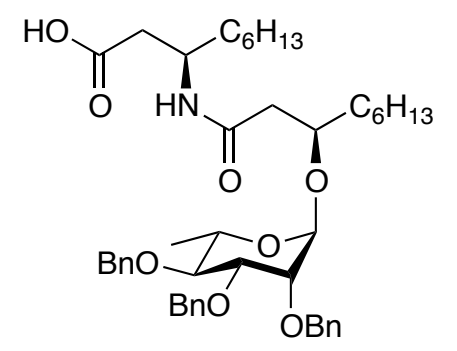

$(R)-3-N$-[2,3,4-tri-O-benzyl- $\alpha$-L-rhamnopyranosyl-(1 $\rightarrow 3)-(R)-3^{\prime}-O$ -

nonanamido]nonanic acid (22): A solution of 21 (50 $\mathrm{mg}, 0.060 \mathrm{mmol})$ in $\mathrm{CH}_{3} \mathrm{OH}-$ water $(4: 1,5.0 \mathrm{~mL})$ was treated with $\mathrm{LiOH} \cdot \mathrm{H}_{2} \mathrm{O}(25 \mathrm{mg}, 0.60 \mathrm{mmol})$ and was stirred at 
$50{ }^{\circ} \mathrm{C}$ overnight. After cooling to r.t., the reaction mixture was acidified to $\mathrm{pH} 5$ by the addition of $1.0 \mathrm{M} \mathrm{HCl}$, diluted with water and then extracted with $\mathrm{CH}_{2} \mathrm{Cl}_{2}$. The organic phase was washed with brine, dried over $\mathrm{Na}_{2} \mathrm{SO}_{4}$, filtered and concentrated. The resulting residue was purified by chromatography (gradient $50 \rightarrow 70 \%$ EtOAc in hexane) to afford 22 (42 mg, 87\% yield) as a colorless oil. $R_{\mathrm{f}} 0.13\left(1: 1\right.$ hexane-EtOAc); $[\alpha]_{\mathrm{D}}=$ -16.4 (c 0.1, $\left.\mathrm{CH}_{2} \mathrm{Cl}_{2}\right) ;{ }^{1} \mathrm{H} \mathrm{NMR}\left(400 \mathrm{MHz}, \mathrm{CDCl}_{3}, \delta_{\mathrm{H}}\right) 7.45-7.28(\mathrm{~m}, 15 \mathrm{H}), 5.51(\mathrm{~d}$, $1 \mathrm{H}, J=9.6 \mathrm{~Hz}), 5.02(\mathrm{~d}, 1 \mathrm{H}, J=10.8 \mathrm{~Hz}), 4.80(\mathrm{~d}, 1 \mathrm{H}, J=11.4 \mathrm{~Hz}), 4.78(\mathrm{~d}, 1 \mathrm{H}, J$ $=12.4 \mathrm{~Hz}), 4.77(\mathrm{~d}, 1 \mathrm{H}, J=2.0 \mathrm{~Hz}), 4.67(\mathrm{~d}, 1 \mathrm{H}, J=10.8 \mathrm{~Hz}), 4.66(\mathrm{~d}, 1 \mathrm{H}, J=11.4$ Hz), 4.65 (d, $1 \mathrm{H}, J=12.4 \mathrm{~Hz}), 4.49-4.39$ (m, $1 \mathrm{H}), 4.26-4.20$ (m, $1 \mathrm{H}), 4.05$ (dd, $1 \mathrm{H}$, $J=8.4,2.8 \mathrm{~Hz}), 3.71-3.65(\mathrm{~m}, 3 \mathrm{H}), 2.56(\mathrm{dd}, 1 \mathrm{H}, J=13.6,3.6 \mathrm{~Hz}), 2.37(\mathrm{dd}, 1 \mathrm{H}, J$ $=14.8,2.4 \mathrm{~Hz}), 2.12(\mathrm{dd}, 1 \mathrm{H}, J=14.8,9.6 \mathrm{~Hz}), 2.07(\mathrm{dd}, 1 \mathrm{H}, J=13.6,10.4 \mathrm{~Hz})$, 1.44-1.36 (m, $4 \mathrm{H}), 1.31(\mathrm{~d}, 3 \mathrm{H}, J=6.4 \mathrm{~Hz}), 1.32-1.21(\mathrm{~m}, 19 \mathrm{H}), 0.90$ (t, $3 \mathrm{H}, J=$ $7.2 \mathrm{~Hz}), 0.86(\mathrm{t}, 3 \mathrm{H}, J=7.2 \mathrm{~Hz}) ;{ }^{13} \mathrm{C} \mathrm{NMR}\left(125 \mathrm{MHz}, \mathrm{CDCl}_{3}, \delta_{\mathrm{C}}\right) 172.6,170.3,139.2$, $138.6,137.3,129.2,128.8,128.71,128.69,128.5,128.4,128.10,128.08,127.8,95.1$, $81.8,80.1,76.7,75.3,73.4,72.4,72.1,67.5,47.5,42.4,40.8,36.5,32.2,32.1,32.0$ 29.8, 29.4, 26.2, 24.8, 22.94, 22.88, 18.1, 14.5, 14.4; HRMS (ESI) m/z [M - H] Calcd for $\mathrm{C}_{45} \mathrm{H}_{62} \mathrm{NO}_{8}$ 744.4481; Found 744.4478. 


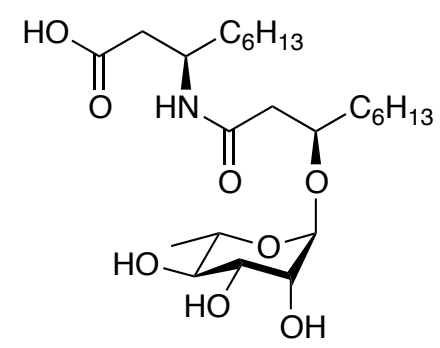

$(R)-3-N$-[ $\alpha$-L-rhamnopyranosyl-( $(1 \rightarrow 3)-(R)-3^{\prime}-O$-nonanamido]nonanic acid (1): A solution of $22(60 \mathrm{mg}, 0.080 \mathrm{mmol})$ in $\mathrm{CH}_{3} \mathrm{OH}(10 \mathrm{~mL})$ and acetic acid $(0.20 \mathrm{~mL})$ was treated with palladium on charcoal $(10 \%, 50 \mathrm{mg})$ and subjected to a hydrogen atmosphere for $20 \mathrm{~h}$. The mixture was filtered through Celite and the filtrate was concentrated. The residue was subjected to chromatography (Iatrobeads 6RS-8060, gradient $10 \rightarrow 40 \% \mathrm{CH}_{3} \mathrm{OH}$ in $\mathrm{CH}_{2} \mathrm{Cl}_{2}$ ) to yield $\mathbf{1}$ (32 $\mathrm{mg}, 87 \%$ yield) as a colorless oil. $[\alpha]_{\mathrm{D}}=-30.5\left(c 0.1, \mathrm{CH}_{2} \mathrm{Cl}_{2}\right) ;{ }^{1} \mathrm{H}$ NMR $\left(700 \mathrm{MHz}, \mathrm{CD}_{3} \mathrm{OD}, \delta_{\mathrm{H}}\right) 4.79(\mathrm{~s}, 1 \mathrm{H}, \mathrm{H}-1)$, 4.25-4.22 (m, 1 H, NHCH), 4.11-4.08 (m, 1 H, $\left.\mathrm{OCHCH}_{2}\right), 3.74$ (s, $\left.1 \mathrm{H}, \mathrm{H}-2\right), 3.68$ (dd, $1 \mathrm{H}, J=9.5,3.5 \mathrm{~Hz}, \mathrm{H}-3), 3.66-3.62$ (m, $1 \mathrm{H}, \mathrm{H}-5), 3.34$ (app t, $1 \mathrm{H}, J=9.5 \mathrm{~Hz}, \mathrm{H}-4$ ), $2.49\left(\mathrm{dd}, 1 \mathrm{H}, J=14.0,7.5 \mathrm{~Hz}, \mathrm{CH}_{2} \mathrm{CONH}\right), 2.40\left(\mathrm{dd}, 1 \mathrm{H}, J=15.0,5.0 \mathrm{~Hz}, \mathrm{CH}_{2} \mathrm{CO}_{2} \mathrm{H}\right)$, $2.36\left(\mathrm{dd}, 1 \mathrm{H}, J=15.0,8.0 \mathrm{~Hz}, \mathrm{CH}_{2} \mathrm{CO}_{2} \mathrm{H}\right), 2.33\left(\mathrm{dd}, 1 \mathrm{H}, J=14.0,5.5 \mathrm{~Hz}, \mathrm{CH}_{2} \mathrm{CONH}\right)$, 1.55-1.43 (m, $\left.4 \mathrm{H}, 2 \times \mathrm{CH}_{2}\right), 1.37-1.27\left(\mathrm{~m}, 16 \mathrm{H}, 8 \times \mathrm{CH}_{2}\right), 1.24(\mathrm{~d}, 3 \mathrm{H}, J=6.0 \mathrm{~Hz}$, H-6), $0.90\left(\mathrm{t}, 3 \mathrm{H}, J=6.4 \mathrm{~Hz}, \mathrm{CH}_{3}\right), 0.89\left(\mathrm{t}, 3 \mathrm{H}, J=6.4 \mathrm{~Hz}, \mathrm{CH}_{3}\right) ;{ }^{13} \mathrm{C}$ NMR $(125$ $\left.\mathrm{MHz}, \mathrm{CD}_{3} \mathrm{OD}, \delta_{\mathrm{C}}\right) 172.9(\mathrm{C}=\mathrm{ONH}), 171.5(\mathrm{C}=\mathrm{OOH}), 99.8(\mathrm{C}-1), 74.9\left(\mathrm{OCHCH}_{2}\right), 74.1$ (C-4), 72.7 (C-2), 72.2 (C-3), $70.5(\mathrm{C}-5), 48.2(\mathrm{NHCH}), 43.0\left(\mathrm{CH}_{2} \mathrm{C}=\mathrm{ONH}\right), 42.1$ $\left(\mathrm{CH}_{2} \mathrm{CO}_{2} \mathrm{H}\right), 36.0\left(\mathrm{CH}_{2}\right), 33.9\left(\mathrm{CH}_{2}\right), 33.03\left(\mathrm{CH}_{2}\right), 32.98\left(\mathrm{CH}_{2}\right), 30.57\left(\mathrm{CH}_{2}\right), 30.31$ $\left(\mathrm{CH}_{2}\right), 27.1\left(\mathrm{CH}_{2}\right), 25.8\left(\mathrm{CH}_{2}\right), 23.7\left(\mathrm{CH}_{2}\right), 17.9(\mathrm{C}-6), 14.4\left(\mathrm{CH}_{3}\right) ; \mathrm{HRMS}(\mathrm{ESI}) \mathrm{m} / \mathrm{z}$ $[\mathrm{M}+\mathrm{Na}]^{+}$Calcd for $\mathrm{C}_{24} \mathrm{H}_{45} \mathrm{NNaO}_{8} 498.3037$; Found 498.3038 . 
<smiles></smiles>

(R)-3-((tert-butyldimethylsilyl)oxy)-1-nonanol (23): Diisobutylaluminium hydride (DIBALH) (10 mL of a 1.0 M solution in hexane, $9.9 \mathrm{mmol})$ was added dropwise to a stirred solution of $11(1.0 \mathrm{~g}, 3.3 \mathrm{mmol})$ in dry THF $(25 \mathrm{~mL})$ that had been pre-cooled to $-78{ }^{\circ} \mathrm{C}$. The resulting mixture was stirred at $-78{ }^{\circ} \mathrm{C}$ for $2 \mathrm{~h}$, then quenched by the addition of $1.0 \mathrm{M} \mathrm{HCl}$. Ethyl acetate was then added to extract the target compound. The organic phase was washed with brine, dried over $\mathrm{Na}_{2} \mathrm{SO}_{4}$, filtered and concentrated. The resulting residue was purified by chromatography (gradient $10 \rightarrow 16 \%$ EtOAc in hexane) to afford 23 ( $0.56 \mathrm{~g}, 65 \%$ yield) as a colorless oil. $R_{\mathrm{f}} 0.55$ (4:1 hexane-EtOAc); $[\alpha]_{\mathrm{D}}=-16.6\left(c 0.6, \mathrm{CH}_{2} \mathrm{Cl}_{2}\right) ;{ }^{1} \mathrm{H} \mathrm{NMR}\left(400 \mathrm{MHz}, \mathrm{CDCl}_{3}, \delta_{\mathrm{H}}\right) 3.91(\mathrm{ddd}, 1 \mathrm{H}, J=12.6$, 6.4, 4.2 Hz), 3.87-3.81 (m, $1 \mathrm{H}), 3.75-3.68(\mathrm{~m}, 1 \mathrm{H}), 2.51(\mathrm{t}, 1 \mathrm{H}, J=5.2 \mathrm{~Hz}), 1.83$ (dddd, $1 \mathrm{H}, J=14.4,8.4,5.0,4.0 \mathrm{~Hz}$ ), 1.65 (ddt $1 \mathrm{H}, J=14.4,6.0,4.4 \mathrm{~Hz}$ ), 1.55-1.50 (m, $2 \mathrm{H}), 1.32-1.26(\mathrm{~m}, 8 \mathrm{H}), 0.90(\mathrm{~s}, 9 \mathrm{H}), 0.89$ (t, $1 \mathrm{H}, J=7.2 \mathrm{~Hz}), 0.10$ (s, $3 \mathrm{H}), 0.08$ $(\mathrm{s}, 3 \mathrm{H}) ;{ }^{13} \mathrm{C} \mathrm{NMR}\left(100 \mathrm{MHz}, \mathrm{CDCl}_{3}, \delta_{\mathrm{C}}\right) 71.7,60.0,37.2,36.4,31.4,29.0,25.4,24.9$, 22.2, 17.6, 13.7, -4.8, -5.1; HRMS (EI) $\mathrm{m} / \mathrm{z}[\mathrm{M}-t \text {-Bu }]^{-}$Calcd for $\mathrm{C}_{11} \mathrm{H}_{25} \mathrm{O}_{2} \mathrm{Si}$ 217.1624; Found 217.1622.



(R)-3-((tert-butyldimethylsilyl)oxy)-1-nonyl iodide (12): To a solution of the alcohol $23(0.57 \mathrm{~g}, 2.1 \mathrm{mmol})$ in THF $(30 \mathrm{~mL})$ at $0{ }^{\circ} \mathrm{C}$ were added $\mathrm{Ph}_{3} \mathrm{P}(0.82 \mathrm{~g}, 3.1 \mathrm{mmol})$, imidazole $(0.43 \mathrm{~g}, 6.3 \mathrm{mmol})$ and $\mathrm{I}_{2}(0.80 \mathrm{~g}, 3.1 \mathrm{mmol})$. After stirring for $1 \mathrm{~h}$, the 
resulting solution was diluted with ether and washed with a saturated $\mathrm{Na}_{2} \mathrm{~S}_{2} \mathrm{O}_{3}$ solution. The aqueous layer was extracted with ether and the organic layer was washed with brine, dried over $\mathrm{Na}_{2} \mathrm{SO}_{4}$, filtered and concentrated. The resulting residue was purified by chromatography (gradient $0 \rightarrow 5 \%$ EtOAc in hexane) to afford $12(0.74 \mathrm{~g}, 91 \%$ yield) as a colorless oil. $R_{\mathrm{f}} 0.84\left(20: 1\right.$ hexane-EtOAc); $[\alpha]_{\mathrm{D}}=-27.1\left(\right.$ c $\left.1.0, \mathrm{CH}_{2} \mathrm{Cl}_{2}\right) ;{ }^{1} \mathrm{H} \mathrm{NMR}$ (400 MHz, $\left.\mathrm{CDCl}_{3}, \delta_{\mathrm{H}}\right) 3.75-3.69(\mathrm{~m}, 1 \mathrm{H}), 3.28-3.17(\mathrm{~m}, 2 \mathrm{H}), 2.00-1.91(\mathrm{~m}, 2 \mathrm{H})$, 1.47-1.43 (m, $2 \mathrm{H}), 1.33-1.26(\mathrm{~m}, 8 \mathrm{H}), 0.90-0.88(\mathrm{~m}, 12 \mathrm{H}), 0.09(\mathrm{~s}, 3 \mathrm{H}), 0.07$ (s, 3 $\mathrm{H}) ;{ }^{13} \mathrm{C} \mathrm{NMR}\left(100 \mathrm{MHz}, \mathrm{CDCl}_{3}, \delta_{\mathrm{C}}\right) 71.8,40.5,36.5,31.4,29.0,25.5,24.5,22.2,17.7$, 13.7, 3.0, -4.7, -4.8; HRMS (EI) $\mathrm{m} / \mathrm{z}[\mathrm{M}-t \text {-Bu] }]^{-}$Calcd for $\mathrm{C}_{11} \mathrm{H}_{24} \mathrm{IOSi} 327.0641$; Found 327.0641 .

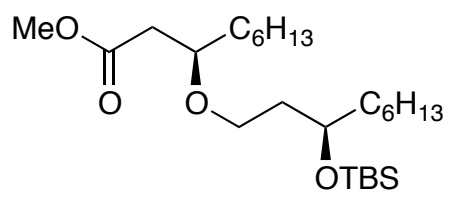

(R)-methyl 3-((R)-3-(((tert-butyldimethylsilyl)oxy)nonyl)oxy)nonanoate (24): To a stirred solution of alcohol $15(0.11 \mathrm{~g}, 0.58 \mathrm{mmol})$ in $\mathrm{CH}_{2} \mathrm{Cl}_{2}(10 \mathrm{~mL})$ was added iodide $12(0.20 \mathrm{~g}, 0.73 \mathrm{mmol})$ and $\mathrm{Ag}_{2} \mathrm{O}(1.7 \mathrm{~g}, 7.3 \mathrm{mmol})$. The reaction mixture was then heated at reflux for 1 day in the absence of light. After cooling to r.t., the solid was separated by filtration through a pad of Celite and the filtrate was concentrated. The resulting residue was purified by chromatography (gradient $0 \rightarrow 5 \%$ EtOAc in hexane) to afford 24 (82 mg, 32\% yield) as a colorless oil. $R_{\mathrm{f}} 0.32\left(20: 1\right.$ hexane-EtOAc); $[\alpha]_{\mathrm{D}}$ $=-6.7\left(c 0.6, \mathrm{CH}_{2} \mathrm{Cl}_{2}\right) ;{ }^{1} \mathrm{H} \mathrm{NMR}\left(400 \mathrm{MHz}, \mathrm{CDCl}_{3}, \delta_{\mathrm{H}}\right) 3.78-3.68(\mathrm{~m}, 2 \mathrm{H}), 3.67(\mathrm{~s}, 3$ H), 3.52-3.47 (m, $2 \mathrm{H}), 2.53(\mathrm{dd}, 1 \mathrm{H}, J=15.0,7.0 \mathrm{~Hz}), 2.40(\mathrm{dd}, 1 \mathrm{H}, J=15.0,5.6$ 
$\mathrm{Hz}), 1.70-1.64(\mathrm{~m}, 2 \mathrm{H}), 1.56-1.26(\mathrm{~m}, 20 \mathrm{H}), 0.90-0.87$ (m, $15 \mathrm{H}), 0.045(\mathrm{~s}, 3 \mathrm{H})$, $0.044(\mathrm{~s}, 3 \mathrm{H}) ;{ }^{13} \mathrm{C} \mathrm{NMR}\left(125 \mathrm{MHz}, \mathrm{CDCl}_{3}, \delta_{\mathrm{C}}\right) 172.3,76.4,69.7,66.2,51.5,39.7,37.4$, $37.3,34.5,31.9,31.8,29.5,29.4,25.9,25.2,25.1,22.64,22.61,18.1,14.10,14.08,-4.4$ -4.5; HRMS (ESI) m/z [M + Na $]^{+}$Calcd for $\mathrm{C}_{25} \mathrm{H}_{52} \mathrm{NaO}_{4} \mathrm{Si}$ 467.3527; Found 467.3521.<smiles>COC(=O)C[C@@H](OCC[C@H](O)c1ccccc1)c1ccccc1</smiles>

(R)-methyl 3-(((R)-3-hydroxynonyl)oxy)nonanoate (7): A solution of $24(0.2 \mathrm{~g}, 0.45$ mmol) in $1.5 \% \mathrm{HCl}$ in $\mathrm{CH}_{3} \mathrm{OH}(40 \mathrm{~mL})$ was stirred for $1 \mathrm{~h}$. The reaction mixture was neutralized by the addition of saturated aqueous $\mathrm{NaHCO}_{3}$, diluted with water and extracted with $\mathrm{CH}_{2} \mathrm{Cl}_{2}$. The organic phase was washed with brine, dried over $\mathrm{Na}_{2} \mathrm{SO}_{4}$, filtered and concentrated. The resulting residue was purified by chromatography (gradient $10 \rightarrow 12 \%$ EtOAc in hexane) to afford $7(0.14 \mathrm{~g}, 91 \%$ yield) as a colorless oil. $R_{\mathrm{f}} 0.39$ (4:1 hexane-EtOAc); $[\alpha]_{\mathrm{D}}=+1.1\left(c\right.$ 0.5, $\left.\mathrm{CH}_{2} \mathrm{Cl}_{2}\right) ;{ }^{1} \mathrm{H} \mathrm{NMR}\left(600 \mathrm{MHz}, \mathrm{CDCl}_{3}\right.$, $\left.\delta_{\mathrm{H}}\right) 3.75-3.70(\mathrm{~m}, 3 \mathrm{H}), 3.69(\mathrm{~s}, 3 \mathrm{H}), 3.62-3.59(\mathrm{~m}, 1 \mathrm{H}), 2.66($ br s, $1 \mathrm{H}), 2.53(\mathrm{dd}, 1$ $\mathrm{H}, J=15.0,7.6 \mathrm{~Hz}), 2.44(\mathrm{dd}, 1 \mathrm{H}, J=15.0,5.0 \mathrm{~Hz}), 1.70-1.62(\mathrm{~m}, 2 \mathrm{H}), 1.58-1.26$ $(\mathrm{m}, 20 \mathrm{H}), 0.88(\mathrm{t}, 3 \mathrm{H}, J=7.0 \mathrm{~Hz}), 0.87$ (t, $3 \mathrm{H}, J=7.0 \mathrm{~Hz}) ;{ }^{13} \mathrm{C}$ NMR $(150 \mathrm{MHz}$, $\left.\mathrm{CDCl}_{3}, \delta_{\mathrm{C}}\right) 172.2,76.9,71.6,68.4,51.7,39.4,37.4,36.6,34.0,31.8,31.7,29.4,29.3$, 25.6, 25.0, 25.1, 22.61, 22.57, 14.08, 14.03; HRMS (ESI) $\mathrm{m} / \mathrm{z}[\mathrm{M}+\mathrm{Na}]^{+}$Calcd for $\mathrm{C}_{19} \mathrm{H}_{38} \mathrm{NaO}_{4}$ 353.2662; Found 353.2655. 


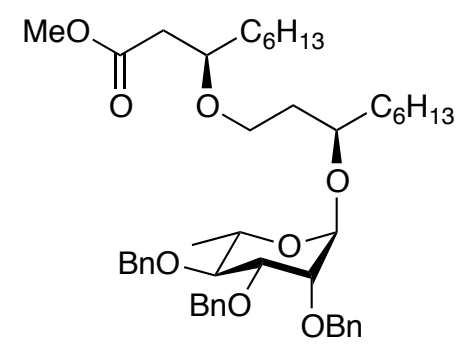

\section{Methyl $(R)-3-O$-[2,3,4-tri- $O$-benzyl- $\alpha$-L-rhamnopyranosyl-(1 $\rightarrow 3)-(R)-3-h y d r o x y$}

nonyl]nonanoate (25): A mixture of acceptor $7 \quad(0.13 \mathrm{~g}, \quad 0.40 \mathrm{mmol})$, trichloroacetimidate donor $5(0.58 \mathrm{~g}, 1.0 \mathrm{mmol})$ and powdered $4 \AA$ molecular sieves was suspended in anhydrous $\mathrm{Et}_{2} \mathrm{O}(10 \mathrm{~mL})$ and stirred for $10 \mathrm{~min}$. The solution was then cooled to $0{ }^{\circ} \mathrm{C}$, and then TMSOTf $(10 \mu \mathrm{L})$ was added. The solution was stirred for $1 \mathrm{~h}$ before $\mathrm{Et}_{3} \mathrm{~N}(0.50 \mathrm{~mL})$ was added and the mixture was filtered. The filtrate was concentrated and the resulting residue was purified by chromatography (gradient $1 \rightarrow 2 \%$ EtOAc in hexane $)$ to afford $25(0.20 \mathrm{~g}, 66 \%$ yield $)$ as a colorless oil; $R_{\mathrm{f}} 0.42(5: 1$ hexane-EtOAc); $[\alpha]_{\mathrm{D}}=+38.9\left(c \quad 0.1, \mathrm{CH}_{2} \mathrm{Cl}_{2}\right) ;{ }^{1} \mathrm{H}$ NMR $\left(600 \mathrm{MHz}, \mathrm{CDCl}_{3}, \delta_{\mathrm{H}}\right)$ 7.38-7.26 (m, $15 \mathrm{H}), 4.94(\mathrm{~d}, 1 \mathrm{H}, J=10.8 \mathrm{~Hz}), 4.78(\mathrm{~d}, 1 \mathrm{H}, J=12.4 \mathrm{~Hz}), 4.77$ (d, 1 $\mathrm{H}, J=2.0 \mathrm{~Hz}), 4.70(\mathrm{~d}, 1 \mathrm{H}, J=12.4 \mathrm{~Hz}), 4.66-4.61(\mathrm{~m}, 3 \mathrm{H}), 3.82(\mathrm{dd}, 1 \mathrm{H}, J=9.4$, 3.2 Hz), 3.76-3.72 (m, $1 \mathrm{H}), 3.70-3.66(\mathrm{~m}, 2 \mathrm{H}), 3.65(\mathrm{~s}, 3 \mathrm{H}), 3.64-3.60(\mathrm{~m}, 2 \mathrm{H})$, 3.50-3.43 (m, $2 \mathrm{H}), 2.50(\mathrm{dd}, 1 \mathrm{H}, J=15.0,7.2 \mathrm{~Hz}), 2.40(\mathrm{dd}, 1 \mathrm{H}, J=15.0,5.6 \mathrm{~Hz})$, $1.73-1.63(\mathrm{~m}, 2 \mathrm{H}), 1.55-1.21(\mathrm{~m}, 23 \mathrm{H}), 0.91(\mathrm{t}, 3 \mathrm{H}, J=7.2 \mathrm{~Hz}), 0.88(\mathrm{t}, 3 \mathrm{H}, J=7.2$ $\mathrm{Hz}) ;{ }^{13} \mathrm{C} \mathrm{NMR}\left(125 \mathrm{MHz}, \mathrm{CDCl}_{3}, \delta_{\mathrm{C}}\right)$ 172.3, 138.75, 138.69, 138.4, 128.36, 128.35, $128.33,128.1,128.0,127.71,127.68,127.58,127.52,96.9,80.7,80.2,76.5,75.34$, $75.24,75.20,72.8,72.3,68.3,66.1,51.5,39.7,35.2,34.4,33.3,31.84,31.80,29.5,29.4$ 25.2, 24.6, 22.7, 22.6, 18.0, 14.14, 14.10; gHSQC 2-D NMR (without ${ }^{1} \mathrm{H}$ decoupling): 
${ }^{1} J_{\mathrm{C} 1, \mathrm{H} 1}=168.9 \mathrm{~Hz}$. HRMS (ESI) m/z $[\mathrm{M}+\mathrm{Na}]^{+}$Calcd for $\mathrm{C}_{46} \mathrm{H}_{66} \mathrm{NaO}_{8}$ 769.4650; Found 769.4645 .

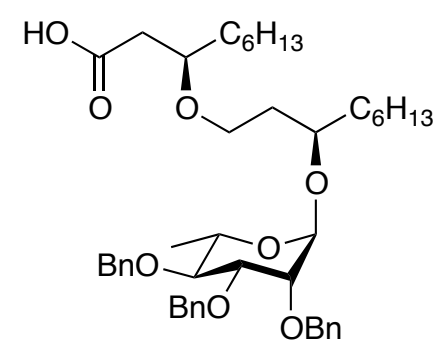

(R)-3-O-[2,3,4-tri-O-benzyl- $\alpha$-L-rhamnopyranosyl-(1 $\rightarrow 3)-(R)-3-$

hydroxynonyl]nonanic acid (26): A solution of 25 (80 mg, $0.11 \mathrm{mmol})$ in $\mathrm{CH}_{3} \mathrm{OH}-$ water $(4: 1,10 \mathrm{~mL})$ was treated with $\mathrm{LiOH} \cdot \mathrm{H}_{2} \mathrm{O}(45 \mathrm{mg}, 1.1 \mathrm{mmol})$ and was stirred at $50{ }^{\circ} \mathrm{C}$ overnight. After cooling to r.t., the reaction mixture was acidified to $\mathrm{pH} 5$ by the addition of $1 \mathrm{M} \mathrm{HCl}$, diluted with water and extracted with $\mathrm{CH}_{2} \mathrm{Cl}_{2}$. The organic phase was washed with brine, dried over $\mathrm{Na}_{2} \mathrm{SO}_{4}$, filtered and concentrated. The resulting residue was purified by chromatography (gradient $20 \rightarrow 25 \%$ EtOAc in hexane) to afford $26(69 \mathrm{mg}, 88 \%$ yield $)$ as a colorless oil. $R_{\mathrm{f}} 0.23(3: 1$ hexane-EtOAc $) ;[\alpha]_{\mathrm{D}}=-16.6(c$ 0.9, $\left.\mathrm{CH}_{2} \mathrm{Cl}_{2}\right) ;{ }^{1} \mathrm{H}$ NMR $\left(500 \mathrm{MHz}, \mathrm{CDCl}_{3}, \delta_{\mathrm{H}}\right) 7.43-7.31(\mathrm{~m}, 15 \mathrm{H}), 5.00(\mathrm{~d}, 1 \mathrm{H}, J=$ $10.5 \mathrm{~Hz}), 4.82-4.66(\mathrm{~m}, 6 \mathrm{H}), 3.93(\mathrm{dd}, 1 \mathrm{H}, J=9.0,3.0 \mathrm{~Hz}), 3.81-3.76(\mathrm{~m}, 1 \mathrm{H})$, 3.73-3.65 (m, $5 \mathrm{H}), 3.46-3.41(\mathrm{~m}, 1 \mathrm{H}), 2.46(\mathrm{dd}, 1 \mathrm{H}, J=15.0,3.5 \mathrm{~Hz}), 2.41(\mathrm{dd}, 1$ $\mathrm{H}, J=15.0,8.0 \mathrm{~Hz}), 1.77-1.59(\mathrm{~m}, 3 \mathrm{H}), 1.48-1.17$ (m, $22 \mathrm{H}), 0.94(\mathrm{t}, 3 \mathrm{H}, J=7.2$ $\mathrm{Hz}), 0.91(\mathrm{t}, 3 \mathrm{H}, J=7.2 \mathrm{~Hz}) ;{ }^{13} \mathrm{C} \mathrm{NMR}\left(125 \mathrm{MHz}, \mathrm{CDCl}_{3}, \delta_{\mathrm{C}}\right) 173.5,138.6,138.3$, $137.8,128.45,128.43,128.38,128.37,128.1,128.0,127.8,127.6,95.2,81.1,79.9,75.9$ $75.1,72.9,72.3,67.9,66.8,39.9,34.9,34.8,32.6,31.80,31.77,29.5,29.2,25.5,24.4$, 
22.63, 22.59, 18.1, 14.12, 14.08; HRMS (ESI) $\mathrm{m} / \mathrm{z}[\mathrm{M}-\mathrm{H}]^{-}$Calcd for $\mathrm{C}_{45} \mathrm{H}_{63} \mathrm{O}_{8}$ 731.4528; Found 731.4529.

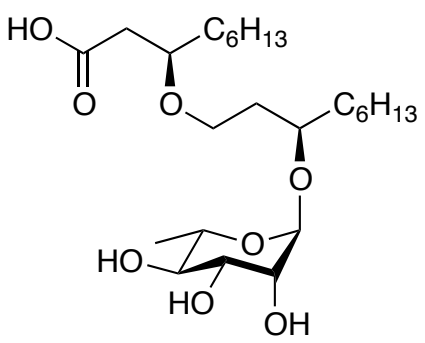

Methyl $(R)-3-O$-[ $\alpha$-L-rhamnopyranosyl-( $\rightarrow 3)-(R)-3-h y d r o x y n o n y l] n o n a n i c$ acid (2): A solution of $26(60 \mathrm{mg}, 0.080 \mathrm{mmol})$ in $\mathrm{CH}_{3} \mathrm{OH}(10 \mathrm{~mL})$, and acetic acid $(0.20$ $\mathrm{mL})$ was treated with palladium on charcoal $(10 \%, 50 \mathrm{mg})$ and subjected to a hydrogen atmosphere for $20 \mathrm{~h}$. The mixture was filtered through Celite and the filtrate was concentrated. The residue was subjected to chromatography (Iatrobeads 6RS-8060, gradient $10 \rightarrow 40 \% \mathrm{CH}_{3} \mathrm{OH}$ in $\mathrm{CH}_{2} \mathrm{Cl}_{2}$ ) to yield 2 (31 $\mathrm{mg}, 82 \%$ yield) as a colorless oil. $[\alpha]_{\mathrm{D}}=-42.8\left(c 0.8, \mathrm{CH}_{2} \mathrm{Cl}_{2}\right) ;{ }^{1} \mathrm{H} \mathrm{NMR}\left(700 \mathrm{MHz}, \mathrm{CD}_{3} \mathrm{OD}, \delta_{\mathrm{H}}\right) 4.75(\mathrm{~d}, 1 \mathrm{H}, J=1.5$ $\mathrm{Hz}, \mathrm{H}-1), 3.75$ (dd, $1 \mathrm{H}, J=3.5,1.5 \mathrm{~Hz}, \mathrm{H}-2), 3.74-3.70\left(\mathrm{~m}, 2 \mathrm{H}, \mathrm{OCHCH}_{2}, \mathrm{OCHCH}_{2}\right)$, 3.66-3.63 (m, $1 \mathrm{H}, \mathrm{H}-5), 3.63$ (dd, $1 \mathrm{H}, J=9.5,3.5 \mathrm{~Hz}, \mathrm{H}-3), 3.59$ (app dt, $1 \mathrm{H}, J=$ 9.5, $6.0 \mathrm{~Hz}, \mathrm{CH}_{2} \mathrm{O}$ ), 3.54 (app dt, $1 \mathrm{H}, J=9.5,6.6 \mathrm{~Hz}, \mathrm{CH}_{2} \mathrm{O}$ ), $3.36(\mathrm{t}, 1 \mathrm{H}, J=9.5 \mathrm{~Hz}$, $\mathrm{H}-4), 2.46\left(\mathrm{dd}, 1 \mathrm{H}, J=15.0,7.5 \mathrm{~Hz}, \mathrm{CH}_{2} \mathrm{CO}\right), 2.39\left(\mathrm{dd}, 1 \mathrm{H}, J=15.0,5.5 \mathrm{~Hz}, \mathrm{CH}_{2} \mathrm{CO}\right.$ ), 1.79-1.70 (m, $\left.2 \mathrm{H}, \mathrm{CH}_{2} \mathrm{CH}_{2} \mathrm{O}\right), 1.57-1.48\left(\mathrm{~m}, 4 \mathrm{H}, 2\right.$ x $\left.\mathrm{CH}_{2}\right), 1.41-1.27$ (m, $16 \mathrm{H}, 8$ x $\left.\mathrm{CH}_{2}\right), 1.25(\mathrm{~d}, 3 \mathrm{H}, J=6.0 \mathrm{~Hz}, \mathrm{H}-6), 0.90\left(\mathrm{t}, 3 \mathrm{H}, J=7.0 \mathrm{~Hz}, \mathrm{CH}_{3}\right), 0.89$ (t, $3 \mathrm{H}, J=$ $\left.7.0 \mathrm{~Hz}, \mathrm{CH}_{3}\right) ;{ }^{13} \mathrm{C}$ NMR $\left(125 \mathrm{MHz}, \mathrm{CD}_{3} \mathrm{OD}, \delta_{\mathrm{C}}\right) 176.1\left(\mathrm{CO}_{2} \mathrm{H}\right), 100.5(\mathrm{C}-1), 78.0$ $\left(\mathrm{CHCH}_{2} \mathrm{CO}_{2} \mathrm{H}\right), 76.3\left(\mathrm{OCHCH}_{2} \mathrm{CH}_{2}\right), 74.0$ (C-4), 72.8 (C-2), 72.5 (C-3), 70.2 (C-5), $67.2\left(\mathrm{OCH}_{2}\right), 40.9\left(\mathrm{CH}_{2} \mathrm{CO}_{2} \mathrm{H}\right), 36.4\left(\mathrm{CH}_{2}\right), 35.6\left(\mathrm{CH}_{2}\right), 34.5\left(\mathrm{CH}_{2}\right), 33.0\left(\mathrm{CH}_{2}\right), 30.58$ 
$\left(\mathrm{CH}_{2}\right), 30.53\left(\mathrm{CH}_{2}\right), 26.4\left(\mathrm{CH}_{2}\right), 25.9\left(\mathrm{CH}_{2}\right), 23.7\left(\mathrm{CH}_{2}\right), 18.1(\mathrm{C}-6), 14.5\left(\mathrm{CH}_{3}\right) ; \mathrm{HRMS}$ (ESI) $\mathrm{m} / \mathrm{z}[\mathrm{M}+\mathrm{Na}]^{+}$Calcd for $\mathrm{C}_{24} \mathrm{H}_{46} \mathrm{NaO}_{8}$ 485.3085; Found 485.3085.<smiles>CCCCCCCCC(=O)N1C(=O)OC[C@H]1Cc1ccccc1</smiles>

(S)-4-Benzyl-3-octanoyloxazolidin-2-one (28): (S)-Benzyl-2-oxazolinone 27 (0.50 g, $2.8 \mathrm{mmol})$ was dissolved in THF $(10 \mathrm{~mL})$ under an argon atmosphere, $n$-BuLi (1.6 M in hexane, $1.2 \mathrm{~mL}, 3.1 \mathrm{mmol}$ ) was added, and the mixture was stirred for $1 \mathrm{~h}$ at $-78{ }^{\circ} \mathrm{C}$. To the mixture was added $n$-octanoyl chloride $(0.50 \mathrm{~g}, 3.1 \mathrm{mmol})$, the mixture was stirred for $2 \mathrm{~h}$ at r.t. before being quenched by the addition of satd. aq. $\mathrm{NH}_{4} \mathrm{Cl}$. The aqueous layer was extracted with EtOAc. The organic phase was washed with brine, dried over $\mathrm{Na}_{2} \mathrm{SO}_{4}$, filtered and concentrated. The resulting residue was purified by chromatography (gradient $10 \rightarrow 16 \%$ EtOA in hexane) to afford 28 ( $0.77 \mathrm{~g}, 90 \%$ yield) as a colorless oil. $R_{\mathrm{f}} 0.37\left(4: 1\right.$ hexane-EtOAc); $[\alpha]_{\mathrm{D}}=+56.4\left(c \quad 0.4, \mathrm{CH}_{2} \mathrm{Cl}_{2}\right) ;{ }^{1} \mathrm{H} \mathrm{NMR}$ $\left(600 \mathrm{MHz}, \mathrm{CDCl}_{3}, \delta_{\mathrm{H}}\right) 7.38-7.31(\mathrm{~m}, 5 \mathrm{H}), 5.15(\mathrm{~d}, 1 \mathrm{H}, J=12.4 \mathrm{~Hz}), 5.13(\mathrm{~d}, 1 \mathrm{H}, J$ $=12.4 \mathrm{~Hz}), 2.93-2.88(\mathrm{~m}, 1 \mathrm{H}), 2.78(\mathrm{dd}, 1 \mathrm{H}, J=16.8,9.0 \mathrm{~Hz}), 2.51(\mathrm{dd}, 1 \mathrm{H}, J=$ 16.8, $5.4 \mathrm{~Hz}), 1.73-1.67(\mathrm{~m}, 1 \mathrm{H}), 1.58-1.52(\mathrm{~m}, 1 \mathrm{H}), 1.37-1.24(\mathrm{~m}, 8 \mathrm{H}), 0.88(\mathrm{t}, 3$ $\mathrm{H}, J=7.2 \mathrm{~Hz}) ;{ }^{13} \mathrm{C} \mathrm{NMR}\left(150 \mathrm{MHz}, \mathrm{CDCl}_{3}, \delta_{\mathrm{C}}\right) 180.9,171.7,135.7,128.5,128.3$, 128.2, 66.5, 41.1, 35.6, 31.7, 31.6, 29.0, 26.8, 22.6, 14.0; HRMS (ESI) m/z [M + Na] ${ }^{+}$ Calcd for $\mathrm{C}_{18} \mathrm{H}_{25} \mathrm{NNaO}_{3} 326.1727$; Found 326.1720. 
<smiles>CC(CC(=O)O)C(=O)N1C(=O)OC[C@H]1Cc1ccccc1</smiles>

Benzyl 4-[(S)-4-benzyl-2-oxooxazolidin-3-yl]-3-hexyl-4-oxobutanoate (29): To a solution of $28(5.0 \mathrm{~g}, 17 \mathrm{mmol})$ in THF $(200 \mathrm{~mL})$ was added LiHMDS (1.0 M in THF, $20 \mathrm{~mL}, 20 \mathrm{mmol}$ ) at $-78^{\circ} \mathrm{C}$, and the mixture was stirred for $20 \mathrm{~min}$ at $0{ }^{\circ} \mathrm{C}$. To this solution was added dropwise benzyl bromoacetate $(4.9 \mathrm{~g}, 21 \mathrm{mmol})$ in THF $(50 \mathrm{~mL})$ over $90 \mathrm{~min}$ at $-78^{\circ} \mathrm{C}$. The reaction mixture was stirred for $30 \mathrm{~min}$ at $0{ }^{\circ} \mathrm{C}$ and then for $2 \mathrm{~h}$ at r.t. before being quenched by the addition of satd. aq. $\mathrm{NH}_{4} \mathrm{Cl}$. The aqueous layer was extracted with EtOAc. The organic phase was washed with brine, dried over $\mathrm{Na}_{2} \mathrm{SO}_{4}$, filtered and concentrated. The resulting residue was purified by chromatography (gradient $4 \rightarrow 10 \%$ EtOAc in hexane) to afford 29 (5.7 g, 76\% yield) as a colorless oil. $R_{\mathrm{f}} 0.41\left(4: 1\right.$ hexane-EtOAc); $[\alpha]_{\mathrm{D}}=+33.1\left(c 0.5, \mathrm{CH}_{2} \mathrm{Cl}_{2}\right) ;{ }^{1} \mathrm{H} \mathrm{NMR}$ $\left(500 \mathrm{MHz}, \mathrm{CDCl}_{3}, \delta_{\mathrm{H}}\right) 7.38-7.22(\mathrm{~m}, 10 \mathrm{H}), 5.12(\mathrm{~s}, 2 \mathrm{H}), 4.66-4.62(\mathrm{~m}, 1 \mathrm{H}), 4.27$ (dddd, $1 \mathrm{H}, J=13.4,7.6,5.8,4.0 \mathrm{~Hz}), 4.16(\mathrm{ddd}, 1 \mathrm{H}, J=9.0,7.6,1.0 \mathrm{~Hz}), 4.12$ (dd, $1 \mathrm{H}, J=9.0,2.8 \mathrm{~Hz}), 3.25(\mathrm{dd}, 1 \mathrm{H}, J=13.6,3.2 \mathrm{~Hz}), 2.99(\mathrm{dd}, 1 \mathrm{H}, J=17.0,10.8$ Hz), $2.63(\mathrm{dd}, 1 \mathrm{H}, J=17.0,4.0 \mathrm{~Hz}), 2.50(\mathrm{dd}, 1 \mathrm{H}, J=13.6,10.0 \mathrm{~Hz}), 1.73-1.67(\mathrm{~m}$, $1 \mathrm{H}), 1.53-1.46(\mathrm{~m}, 1 \mathrm{H}), 1.37-1.24(\mathrm{~m}, 8 \mathrm{H}), 0.89(\mathrm{t}, 3 \mathrm{H}, J=7.2 \mathrm{~Hz}) ;{ }^{13} \mathrm{C} \mathrm{NMR}(125$ $\left.\mathrm{MHz}, \mathrm{CDCl}_{3}, \delta_{\mathrm{C}}\right) 175.8,171.9,153.1,135.79,135.77,129.5,128.9,128.6,128.32$, $128.27,127.2,66.6,65.9,55.6,39.2,37.3,35.9,32.2,31.6,29.2,26.8,22.6,14.0$ HRMS (ESI) m/z [M + Na] $]^{+}$Calcd for $\mathrm{C}_{27} \mathrm{H}_{33} \mathrm{NNaO}_{5}$ 474.2251; Found 474.2248. 
<smiles>CC(CC(=O)O)C(=O)O</smiles>

(R)-3-Benzyloxycarbonyl-2-hexylpropanoic acid (30): To a solution of 29 (1.0 g, 2.2 mmol) in THF- $\mathrm{H}_{2} \mathrm{O}(48 \mathrm{~mL}: 12 \mathrm{~mL})$ were added $30 \%$ aq. $\mathrm{H}_{2} \mathrm{O}_{2}(1.3 \mathrm{~g}, 11 \mathrm{mmol})$ and a solution of $\mathrm{LiOH} \cdot \mathrm{H}_{2} \mathrm{O}(0.14 \mathrm{~g}, 3.3 \mathrm{mmol})$ in $\mathrm{H}_{2} \mathrm{O}(6.0 \mathrm{~mL})$. The mixture was stirred for $2 \mathrm{~h}$ at $0{ }^{\circ} \mathrm{C}$, then acidified with $1 \mathrm{M} \mathrm{HCl}$ and warmed to r.t.. The aqueous layer was extracted with EtOAc. The organic phase was washed with satd. aq. $\mathrm{NaHCO}_{3}$, brine, dried over $\mathrm{Na}_{2} \mathrm{SO}_{4}$, filtered and concentrated. The resulting residue was purified by chromatography ( $4 \rightarrow 25 \%$ EtOAc in hexane) to afford 30 (0.42 g, 65\% yield) as a colorless oil. $R_{\mathrm{f}} 0.20\left(3: 1\right.$ hexane-EtOAc); $[\alpha]_{\mathrm{D}}=+9.8\left(c 1.0, \mathrm{CH}_{2} \mathrm{Cl}_{2}\right) ;{ }^{1} \mathrm{H}$ NMR $(500$ $\left.\mathrm{MHz}, \mathrm{CDCl}_{3}, \delta_{\mathrm{H}}\right) 7.41-7.33(\mathrm{~m}, 5 \mathrm{H}), 5.18(\mathrm{~d}, 1 \mathrm{H}, J=12.5 \mathrm{~Hz}), 5.15(\mathrm{~d}, 1 \mathrm{H}, J=12.5$ Hz), 2.96-2.91 (m, $1 \mathrm{H}), 2.81(\mathrm{dd}, 1 \mathrm{H}, J=17.0,9.0 \mathrm{~Hz}), 2.54(\mathrm{dd}, 1 \mathrm{H}, J=17.0,5.0$ Hz), 1.76-1.69 (m, $1 \mathrm{H}), 1.61-1.55$ (m, $1 \mathrm{H}), 1.39-1.27$ (m, $8 \mathrm{H}), 0.91$ (t, $3 \mathrm{H}, J=7.2$ $\mathrm{Hz}) ;{ }^{13} \mathrm{C} \mathrm{NMR}\left(125 \mathrm{MHz}, \mathrm{CDCl}_{3}, \delta_{\mathrm{C}}\right) 180.6,171.8,135.7,128.6,128.3,128.2,66.6$, 41.0, 35.7, 31.7, 31.60, 31.57, 29.1, 26.8, 22.61, 22.57, 14.0; HRMS (ESI) m/z [M H] $]^{-}$Calcd for $\mathrm{C}_{17} \mathrm{H}_{23} \mathrm{O}_{4}$ 291.1602; Found 291.1598.<smiles>CCC(CO)CC(=O)OBr</smiles>

(R)-Benzyl-3-hydroxymethyl-nonanoate (31): To a solution of acid 30 (0.86 g, 2.9 mmol) in dry THF cooled in an ice-salt bath, $\mathrm{BH}_{3} \cdot \mathrm{Me}_{2} \mathrm{~S}$ complex $(0.35 \mathrm{~mL}, 3.5 \mathrm{mmol})$ was added by syringe. The mixture was warmed to r.t. and stirred for $24 \mathrm{~h}$. The reaction was quenched by water $(0.30 \mathrm{~mL})$ with caution at $0{ }^{\circ} \mathrm{C}$, before solid $\mathrm{K}_{2} \mathrm{CO}_{3}(0.50 \mathrm{~g})$ 
was added and the mixture was stirred for $2 \mathrm{~min}$. The aqueous layer was extracted with $\mathrm{Et}_{2} \mathrm{O}$. The organic phase was washed with brine, dried over $\mathrm{Na}_{2} \mathrm{SO}_{4}$, filtered and concentrated. The resulting residue was purified by chromatography (gradient $4 \rightarrow 25 \%$ EtOAc in hexane) to afford $31(0.74 \mathrm{~g}, 90 \%$ yield $)$ as a colorless oil. $R_{\mathrm{f}} 0.33(3: 1$ hexane-EtOAc); $[\alpha]_{\mathrm{D}}=-1.2\left(c \quad 0.6, \mathrm{CH}_{2} \mathrm{Cl}_{2}\right) ;{ }^{1} \mathrm{H}$ NMR $\left(500 \mathrm{MHz}, \mathrm{CDCl}_{3}, \delta_{\mathrm{H}}\right)$ 7.40-7.33 (m, $5 \mathrm{H}), 5.15$ (s, $2 \mathrm{H}), 3.67$ (ddd, $1 \mathrm{H}, J=11.0,5.5,5.0 \mathrm{~Hz}), 3.52$ (app dt, $1 \mathrm{H}, J=11.0,6.5 \mathrm{~Hz}), 2.48(\mathrm{dd}, 1 \mathrm{H}, J=15.5,7.5 \mathrm{~Hz}), 2.42(\mathrm{dd}, 1 \mathrm{H}, J=15.5,5.5$ Hz), 2.09-2.04 (m, $1 \mathrm{H}), 1.75$ (t, $1 \mathrm{H}, J=5.5 \mathrm{~Hz}), 1.41-1.24(\mathrm{~m}, 10 \mathrm{H}), 0.89$ (t, $3 \mathrm{H}, J$ $=7.2 \mathrm{~Hz}) ;{ }^{13} \mathrm{C} \mathrm{NMR}\left(125 \mathrm{MHz}, \mathrm{CDCl}_{3}, \delta_{\mathrm{C}}\right) 173.6,135.9,128.6,128.28,128.27,66.3$, 65.7, 37.9, 36.9, 31.7, 31.2, 29.4, 26.8, 22.6, 14.0; HRMS (ESI) m/z [M + Na $]^{+}$Calcd for $\mathrm{C}_{17} \mathrm{H}_{26} \mathrm{NaO}_{3} 301.1774$; Found 301.1766.<smiles>C[C@H](C=O)CC(=O)OCc1ccccc1</smiles>

(R)-Benzyl-3-carbonyl-nonanoate (13): To a mixture of $\mathbf{3 1}(0.18 \mathrm{~g}, 0.65 \mathrm{mmol})$ and $4 \AA$ molecular sieves in dry $\mathrm{CH}_{2} \mathrm{Cl}_{2}(5.0 \mathrm{~mL})$ were added NMO $(0.11 \mathrm{~g}, 0.98 \mathrm{mmol})$ and TPAP (22 mg, $0.065 \mathrm{mmol}$ ) at r.t. The mixture was stirred for $6 \mathrm{~h}$ and then the mixture was concentrated and the crude product was purified by chromatography (gradient $10 \rightarrow 20 \%$ EtOAc in hexane) to afford $\mathbf{1 3}(0.15 \mathrm{~g}, 85 \%$ yield) as a colorless oil. ${ }^{1} \mathrm{H}$ NMR (400 MHz, $\left.\mathrm{CDCl}_{3}, \delta_{\mathrm{H}}\right) 9.71(\mathrm{~d}, 1 \mathrm{H}, J=1.0 \mathrm{~Hz}), 7.40-7.30(\mathrm{~m}, 5 \mathrm{H}), 5.13$ (s, $2 \mathrm{H}), 2.88-2.81(\mathrm{~m}, 1 \mathrm{H}), 2.76(\mathrm{dd}, 1 \mathrm{H}, J=16.5,8.0 \mathrm{~Hz}), 2.45(\mathrm{dd}, 1 \mathrm{H}, J=16.5$, 5.0 Hz), 1.77-1.69 (m, $1 \mathrm{H}), 1.52-1.44(\mathrm{~m}, 1 \mathrm{H}), 1.37-1.23(\mathrm{~m}, 8 \mathrm{H}), 1.41-1.24(\mathrm{~m}$, $10 \mathrm{H}), 0.88(\mathrm{t}, 3 \mathrm{H}, J=7.2 \mathrm{~Hz}) ;{ }^{13} \mathrm{C} \mathrm{NMR}\left(100 \mathrm{MHz}, \mathrm{CDCl}_{3}, \delta_{\mathrm{C}}\right) 202.5,171.4,135.3$, 
$128.2,127.9,127.8,66.2,47.3,32.7,31.2,28.8,28.2,26.3,22.1,13.6$. Given the instability of this compound to oxidation, no mass spec data could be obtained.

Procedure for determining the enantiomeric excess of compound 13: To a solution of 13 (21 mg $0.077 \mathrm{mmol})$ in $\mathrm{CH}_{2} \mathrm{Cl}_{2}(2.0 \mathrm{~mL}),(2 S, 4 S)-(+)$-pentanediol $(20 \mathrm{mg}, 0.19$ mmol) and $p$-toluenesulfonic acid monohydrate $(3.5 \mathrm{mg}, 0.019 \mathrm{mmol})$ were added. The solution was stirred at $\mathrm{rt}$ for $2 \mathrm{~h}$. The mixture was concentrated under reduced pressure. The enantiomeric excess was determined by ${ }^{1} \mathrm{H}$ NMR analysis of the resulting acetal S4. ${ }^{1} \mathrm{H}$ NMR $\left(600 \mathrm{MHz}, \mathrm{CDCl}_{3}, \delta_{\mathrm{H}}\right)$ of $\mathbf{S 4}: 7.40-7.30(\mathrm{~m}, 5 \mathrm{H}), 5.13(\mathrm{~s}, 2 \mathrm{H}), 4.27-4.19$ (m, 2H, overlap with H-2 of (2S,4S)-(+)-pentanediol), 3.91-3.85 (m, $1 \mathrm{H}), 2.57$ (dd, 1 $\mathrm{H}, J=15.5,6.7 \mathrm{~Hz}), 2.32(\mathrm{dd}, 1 \mathrm{H}, J=15.5,6.7 \mathrm{~Hz}), 2.12-2.08(\mathrm{~m}, 1 \mathrm{H}), 1.76(\mathrm{ddd}, 1$ $\mathrm{H}, J=13.2,11.7,6.1 \mathrm{~Hz}), 1.55-1.50(\mathrm{~m}, 1 \mathrm{H}), 1.31(\mathrm{~d}, 3 \mathrm{H} J=6.7 \mathrm{~Hz}), 1.31-1.27(\mathrm{~m}$, $10 \mathrm{H}), 1.15(\mathrm{~d}, 3 \mathrm{H} J=6.1 \mathrm{~Hz}), 0.88(\mathrm{t}, 3 \mathrm{H}, J=7.2 \mathrm{~Hz})$.<smiles>O=C(CC(/C=C\CC(Cc1ccccc1)OCc1ccccc1)c1ccccc1)Oc1ccccc1</smiles>

(3R,7R,Z)-Benzyl 7-((tert-butyldimethylsilyl)oxy)-3-hexyltridec-4-enoate $\quad(33)$ : Iodide 12 (0.60 g, $1.6 \mathrm{mmol}), \mathrm{PPh}_{3}(0.61 \mathrm{~g}, 2.3 \mathrm{mmol})$ and DIEA $(1.4 \mathrm{~g}, 11 \mathrm{mmol})$ were heated in a sealed flask at $90{ }^{\circ} \mathrm{C}$ for $12 \mathrm{~h}$. After cooling to r.t., DIEA was removed with n-pentane in vacuo and the residue was resuspended in dry $n$-pentane. After $1 \mathrm{~min}$, the $n$-pentane was removed by pipette (repeated twice). After removal of the remaining solvent in vacuo the residue was dissolved in $30 \mathrm{~mL}$ of THF and LiHMDS (1.0 M in THF, $1.5 \mathrm{~mL}, 1.5 \mathrm{mmol}$ ) were added at $-78{ }^{\circ} \mathrm{C}$. After stirring for $15 \mathrm{~min}$, a solution of 
$1.5 \mathrm{~mL}$ of HMPA and $1.5 \mathrm{~mL}$ of THF was added dropwise at $-78^{\circ} \mathrm{C}$ followed by the dropwise addition of a solution of aldehyde $13(0.36 \mathrm{~g}, 1.3 \mathrm{mmol})$ in THF $(5.0 \mathrm{~mL})$. The mixture was stirred for $15 \mathrm{~min}$ at $-78{ }^{\circ} \mathrm{C}$ and for $1 \mathrm{~h}$ at r.t. before the addition of satd. aq. $\mathrm{NaHCO}_{3}$ solution. Then, the mixture was warmed to r.t. and the solution was extracted with ether. The organic phase were dried $\left(\mathrm{Na}_{2} \mathrm{SO}_{4}\right)$, filtered and concentrated. The resulting residue was purified by chromatography (gradient $1 \rightarrow 4 \%$ EtOAc in hexane) to afford $33(0.45 \mathrm{~g}, 67 \%$ yield $)$ as a colorless oil. $R_{\mathrm{f}} 0.40$ (20:1 hexane-EtOAc); $[\alpha]_{\mathrm{D}}=+10.8\left(c 0.6, \mathrm{CH}_{2} \mathrm{Cl}_{2}\right) ;{ }^{1} \mathrm{H} \mathrm{NMR}\left(600 \mathrm{MHz}, \mathrm{CDCl}_{3}, \delta_{\mathrm{H}}\right) 7.37-7.31(\mathrm{~m}, 5 \mathrm{H}), 5.46$ (appdt, $1 \mathrm{H}, J=10.5,7.0 \mathrm{~Hz}), 5.18($ apptt, $1 \mathrm{H}, J=10.5,2.0 \mathrm{~Hz}), 5.11(\mathrm{~d}, 1 \mathrm{H}, J=12.5$ Hz), 5.06 (d, $1 \mathrm{H}, J=12.5 \mathrm{~Hz}), 3.67-3.63$ (m, $1 \mathrm{H}), 2.86-2.79$ (m, $1 \mathrm{H}), 2.38$ (dd, $1 \mathrm{H}$, $J=14.5,6.5 \mathrm{~Hz}), 2.25(\mathrm{dd}, 1 \mathrm{H}, J=14.5,8.0 \mathrm{~Hz}), 2.24-2.21(\mathrm{~m}, 1 \mathrm{H}), 2.17-2.12(\mathrm{~m}$, $1 \mathrm{H}), 1.42-1.19(\mathrm{~m}, 20 \mathrm{H}), 0.89-0.86(\mathrm{~m}, 15 \mathrm{H}), 0.04(\mathrm{~s}, 6 \mathrm{H}) ;{ }^{13} \mathrm{C}$ NMR (125 MHz, $\left.\mathrm{CDCl}_{3}, \delta_{\mathrm{C}}\right) 172.4,136.1,133.9,128.5,128.2,128.1,127.1,72.2,66.1,40.7,36.9,35.4$ $34.6,31.9,31.8,29.5,29.4,27.2,25.9,25.4,22.7,22.6,18.1,14.11,14.09,-4.3,-4.5$; HRMS (ESI) m/z [M + Na] ${ }^{+}$Calcd for $\mathrm{C}_{32} \mathrm{H}_{56} \mathrm{NaO}_{3} \mathrm{Si}$ 539.3891; Found 539.3883.<smiles>O=C(CC(/C=C\C[C@H](O)c1ccccc1)c1ccccc1)OCc1ccccc1</smiles>

(3R,7R,Z)-benzyl 3-hexyl-7-hydroxytridec-4-enoate (8): A solution of 33 (0.50 g, $0.39 \mathrm{mmol})$ in $1.5 \% \mathrm{HCl}$ in $\mathrm{CH}_{3} \mathrm{OH}(30 \mathrm{~mL})$ was stirred for $1 \mathrm{~h}$. The reaction mixture was neutralized by the addition of satd. aq. $\mathrm{NaHCO}_{3}$, diluted with water and extracted with $\mathrm{CH}_{2} \mathrm{Cl}_{2}$. The organic phase was washed with brine, dried over $\mathrm{Na}_{2} \mathrm{SO}_{4}$, filtered 
and concentrated. The resulting residue was purified by chromatography (gradient $10 \rightarrow 12 \%$ EtOAc in hexane) to afford $8(0.35 \mathrm{~g}, 90 \%$ yield $)$ as a colorless oil. $R_{\mathrm{f}} 0.41$ (5:1 hexane-EtOAc); $[\alpha]_{\mathrm{D}}=+3.6\left(c \quad 0.4, \mathrm{CH}_{2} \mathrm{Cl}_{2}\right) ;{ }^{1} \mathrm{H}$ NMR $\left(600 \mathrm{MHz}, \mathrm{CDCl}_{3}, \delta_{\mathrm{H}}\right)$ 7.39-7.32 (m, $5 \mathrm{H}), 5.48$ (app dt, $1 \mathrm{H}, J=10.5,7.5 \mathrm{~Hz}$ ), 5.29 (app tt, $1 \mathrm{H}, J=10.5,1.5$ $\mathrm{Hz}), 5.11(\mathrm{~d}, 1 \mathrm{H}, J=12.5 \mathrm{~Hz}), 5.09(\mathrm{~d}, 1 \mathrm{H}, J=12.5 \mathrm{~Hz}), 3.65-3.61(\mathrm{~m}, 1 \mathrm{H})$, 2.91-2.86 (m, $1 \mathrm{H}), 2.44(\mathrm{dd}, 1 \mathrm{H}, J=15.0,5.5 \mathrm{~Hz}), 2.27(\mathrm{dd}, 1 \mathrm{H}, J=15.0,9.0 \mathrm{~Hz})$, 2.27-2.18 (m, $2 \mathrm{H}), 1.46-1.24(\mathrm{~m}, 20 \mathrm{H}), 0.90$ (t, $3 \mathrm{H}, J=7.2 \mathrm{~Hz}), 0.88$ (t, $3 \mathrm{H}, J=7.2$ $\mathrm{Hz}) ;{ }^{13} \mathrm{C} \mathrm{NMR}\left(100 \mathrm{MHz}, \mathrm{CDCl}_{3}, \delta_{\mathrm{C}}\right) 172.6,136.0,135.7,128.5,128.3,128.2,126.3$, $71.3,66.2,40.6,36.7,35.43,35.39,34.4,31.9,31.8,29.4,27.1,25.8,22.6,14.09,14.08$; HRMS (ESI) m/z [M + Na $]^{+}$Calcd for $\mathrm{C}_{26} \mathrm{H}_{42} \mathrm{NaO}_{3}$ 425.3026; Found 425.3024.

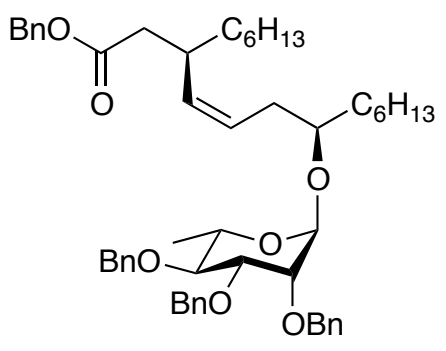

(3R,7R,4Z)-Benzyl-3-n-hexyl-7-(2,3,4-tri-O-benzyl- $\alpha$-L-rhamnopyranosyl)-tridec-

4-enoate (34): A mixture of acceptor $8(0.13 \mathrm{~g}, 0.32 \mathrm{mmol})$, trichloroacetimidate donor $5(0.33 \mathrm{~g}, 0.58 \mathrm{mmol})$ and powdered $4 \AA$ molecular sieves was suspended in anhydrous $\mathrm{Et}_{2} \mathrm{O}(8.0 \mathrm{~mL})$ and stirred for $10 \mathrm{~min}$. The solution was then cooled to $0{ }^{\circ} \mathrm{C}$ and then TMSOTf $(10 \mu \mathrm{L})$ was added. The solution was stirred for $1 \mathrm{~h}$ before $\mathrm{Et}_{3} \mathrm{~N}(0.50 \mathrm{~mL})$ was added and the mixture was filtered. The filtrate was concentrated and the resulting residue was purified by chromatography (gradient $5 \rightarrow 10 \%$ EtOAc in hexane) to afford $34(0.17 \mathrm{~g}, 64 \%$ yield $)$ as a colorless oil; $R_{\mathrm{f}} 0.55(5: 1$ hexane-EtOAc $) ;[\alpha]_{\mathrm{D}}=+4.9(c$ 
0.2, $\left.\mathrm{CH}_{2} \mathrm{Cl}_{2}\right) ;{ }^{1} \mathrm{H} \mathrm{NMR}\left(400 \mathrm{MHz}, \mathrm{CDCl}_{3}, \delta_{\mathrm{H}}\right) 7.39-7.28(\mathrm{~m}, 20 \mathrm{H}), 5.40$ (app dt, $1 \mathrm{H}$, $J=11.0,7.0 \mathrm{~Hz}), 5.18($ app t, $1 \mathrm{H}, J=11.0 \mathrm{~Hz}), 5.11(\mathrm{~d}, 1 \mathrm{H}, J=12.0 \mathrm{~Hz}), 5.07$ (d, 1 $\mathrm{H}, J=12.0 \mathrm{~Hz}), 4.95(\mathrm{~d}, 1 \mathrm{H}, J=11.0 \mathrm{~Hz}), 4.79(\mathrm{~d}, 1 \mathrm{H}, J=12.5 \mathrm{~Hz}), 4.78(\mathrm{~d}, 1 \mathrm{H}, J$ $=1.5 \mathrm{~Hz}), 4.70(\mathrm{~d}, 1 \mathrm{H}, J=12.5 \mathrm{~Hz}), 4.68-4.61(\mathrm{~m}, 3 \mathrm{H}), 3.84(\mathrm{dd}, 1 \mathrm{H}, J=9.5,3.0$ $\mathrm{Hz}), 3.83-3.77(\mathrm{~m}, 1 \mathrm{H}), 3.72(\mathrm{dd}, 1 \mathrm{H}, J=3.0,1.5 \mathrm{~Hz}), 3.62(\operatorname{app} \mathrm{t}, 1 \mathrm{H}, J=9.5 \mathrm{~Hz})$, 3.55-3.49 (m, $1 \mathrm{H}), 2.85-2.77(\mathrm{~m}, 1 \mathrm{H}), 2.38(\mathrm{dd}, 1 \mathrm{H}, J=15.0,6.5 \mathrm{~Hz}), 2.33-2.14$ (m, $3 \mathrm{H}), 1.39-1.12(\mathrm{~m}, 23 \mathrm{H}), 0.92(\mathrm{t}, 3 \mathrm{H}, J=7.2 \mathrm{~Hz}), 0.87(\mathrm{t}, 3 \mathrm{H}, J=7.2 \mathrm{~Hz}) ;{ }^{13} \mathrm{C}$ NMR (100 MHz, $\left.\mathrm{CDCl}_{3}, \delta_{\mathrm{C}}\right) 172.4,138.8,138.7,138.4,136.1,134.3,128.5,128.3$, $128.2,128.1,128.06,128.04,127.7,127.66,127.59,127.51,126.6,97.7,80.7,80.3$, $78.1,75.4,75.2,72.8,72.3,68.3,66.1,40.6,35.3,34.5,33.4,31.9,31.8,29.5,29.4$, 27.2, 25.1, 22.71, 22.66, 17.9, 14.15, 14.10; gHSQC 2-D NMR (without ${ }^{1} \mathrm{H}$ decoupling): ${ }^{1} J_{\mathrm{C} 1, \mathrm{H} 1}=168.8 \mathrm{~Hz}$. HRMS (ESI) $\mathrm{m} / \mathrm{z}[\mathrm{M}+\mathrm{Na}]^{+}$Calcd for $\mathrm{C}_{53} \mathrm{H}_{70} \mathrm{NaO}_{7}$ 841.5014; Found 841.5000 .

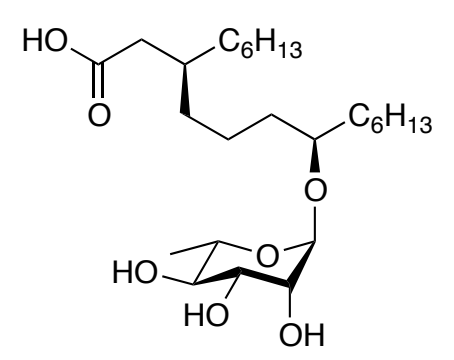

$(3 R, 7 R, 4 Z)-B e n z y l-3-n-h e x y l-7-(\alpha-L-r h a m n o p y r a n o s y l)-t r i d e c-4-e n o a t e \quad(3): \quad A$ solution of $34(70 \mathrm{mg}, 0.085 \mathrm{mmol})$ in $\mathrm{CH}_{3} \mathrm{OH}(10 \mathrm{~mL})$ and acetic acid $(0.20 \mathrm{~mL})$ was treated with palladium on charcoal $(10 \%, 50 \mathrm{mg})$ and subjected to a hydrogen atmosphere for $20 \mathrm{~h}$. The mixture was filtered through Celite and the filtrate was concentrated. The residue was subjected to chromatography (Iatrobeads 6RS-8060, 
gradient $10 \rightarrow 40 \% \mathrm{CH}_{3} \mathrm{OH}$ in $\mathrm{CH}_{2} \mathrm{Cl}_{2}$ ) to yield 3 (34 mg, $86 \%$ yield) as a colorless oil. $[\alpha]_{\mathrm{D}}=-38.6\left(c 1.1, \mathrm{CH}_{2} \mathrm{Cl}_{2}\right) ;{ }^{1} \mathrm{H} \mathrm{NMR}\left(700 \mathrm{MHz}, \mathrm{CD}_{3} \mathrm{OD}, \delta_{\mathrm{H}}\right) 4.75(\mathrm{~d}, 1 \mathrm{H}, J=1.5$ Hz, H-1), 3.75 (dd, $1 \mathrm{H}, J=3.5,1.5 \mathrm{~Hz}, \mathrm{H}-2), 3.67$ (app dq, $1 \mathrm{H}, J=9.5,6.0 \mathrm{~Hz}, \mathrm{H}-5)$, $3.63(\mathrm{dd}, 1 \mathrm{H}, J=9.5,3.5 \mathrm{~Hz}, \mathrm{H}-3), 3.61-3.58\left(\mathrm{~m}, 1 \mathrm{H}, \mathrm{OCHCH}_{2}\right), 3.37$ (app t, $1 \mathrm{H}, J$ $=9.5 \mathrm{~Hz}, \mathrm{H}-4), 2.23\left(\mathrm{dd}, 1 \mathrm{H}, J=15.0,6.5 \mathrm{~Hz}, \mathrm{CH}_{2} \mathrm{CO}\right), 2.19(\mathrm{dd}, 1 \mathrm{H}, J=15.0,7.0$ $\left.\mathrm{Hz}, \mathrm{CH}_{2} \mathrm{CO}\right), 1.85-1.81\left(\mathrm{~m}, 1 \mathrm{H}, \mathrm{CHCH}_{2} \mathrm{CO}_{2} \mathrm{H}\right), 1.53-1.26\left(\mathrm{~m}, 26 \mathrm{H}, 13 \times \mathrm{CH}_{2}\right), 1.24$ (d, $3 \mathrm{H}, J=6.0 \mathrm{~Hz}, \mathrm{H}-6), 0.90$ (t, $3 \mathrm{H}, J=7.0 \mathrm{~Hz}, \mathrm{CH}_{3}$ ), 0.89 (t, $3 \mathrm{H}, J=7.0 \mathrm{~Hz}, \mathrm{CH}_{3}$ ); ${ }^{13} \mathrm{C} \mathrm{NMR}\left(125 \mathrm{MHz}, \mathrm{CD}_{3} \mathrm{OD}, \delta_{\mathrm{C}}\right) 177.5(\mathrm{C}=\mathrm{O}), 100.6(\mathrm{C}-1), 78.9\left(\mathrm{OCHCH}_{2} \mathrm{CH}_{2}\right), 74.0$ (C-4), 72.9 (C-2), 72.5 (C-3), $70.2(\mathrm{C}-5), 40.2\left(\mathrm{CH}_{2} \mathrm{CO}_{2} \mathrm{H}\right), 36.2\left(\mathrm{CHCH}_{2} \mathrm{CO}_{2} \mathrm{H}\right), 36.0$ $\left(\mathrm{CH}_{2}\right), 35.4\left(\mathrm{CH}_{2}\right), 35.1\left(\mathrm{CH}_{2}\right), 34.4\left(\mathrm{CH}_{2}\right), 33.01\left(\mathrm{CH}_{2}\right), 33.00\left(\mathrm{CH}_{2}\right), 30.68\left(\mathrm{CH}_{2}\right)$, $30.63\left(\mathrm{CH}_{2}\right), 27.7\left(\mathrm{CH}_{2}\right), 26.0\left(\mathrm{CH}_{2}\right), 23.73\left(\mathrm{CH}_{2}\right), 23.70\left(\mathrm{CH}_{2}\right), 18.1(\mathrm{C}-6), 14.46$ $\left(\mathrm{CH}_{3}\right), 14.45\left(\mathrm{CH}_{3}\right)$; HRMS (ESI) m/z $[\mathrm{M}+\mathrm{Na}]^{+}$Calcd for $\mathrm{C}_{25} \mathrm{H}_{48} \mathrm{NaO}_{7} 483.3292$; Found 483.3293.

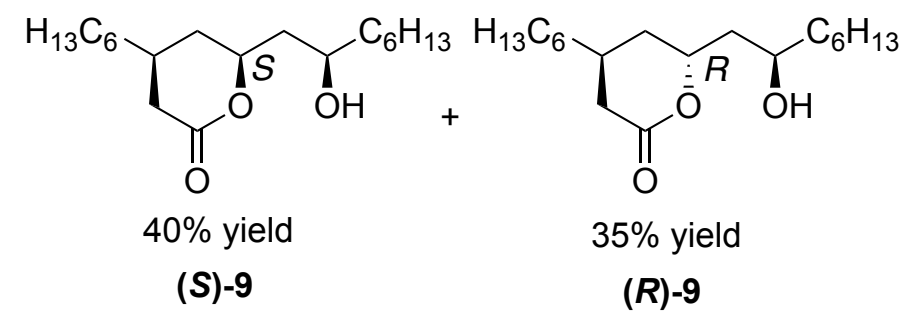

$(4 S, 6 S)-4-h e x y l-6-((R)-2-h y d r o x y o c t y l) t e t r a h y d r o-2 H-p y r a n-2-o n e \quad((S)-9)$ and

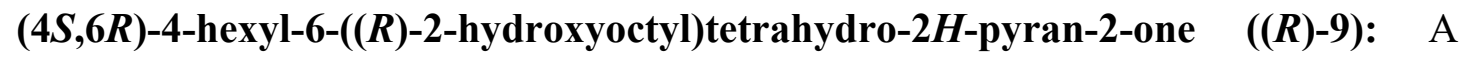
mixture of alcohol $8(0.33 \mathrm{~g}, 0.83 \mathrm{mmol})$ and $\left(\mathrm{HMe}_{2} \mathrm{Si}\right)_{2} \mathrm{NH}(0.3 \mathrm{~mL}, 1.7 \mathrm{mmol})$ was stirred at r.t. overnight. The excess disilazane was removed under vacuum to provide the desired silane as a colorless oil. The silane was then dissolved in anhydrous toluene 
$(9.0 \mathrm{~mL})$ and cooled to $0{ }^{\circ} \mathrm{C}$. To the resulting solution was slowly added Karstedt's catalyst ( $82 \mathrm{mg}, 0.0041 \mathrm{mmol})$ at $0{ }^{\circ} \mathrm{C}$ under argon. The resulting mixture was stirred at $0{ }^{\circ} \mathrm{C}$ for $3 \mathrm{~h}$ and then warmed to r.t. The solvent was removed under vacuum to provide crude $\mathbf{2 . 1 1 8}$ as a light yellow oil. The compound was then dissolved in THF$\mathrm{CH}_{3} \mathrm{OH}(7.0 \mathrm{~mL}: 7.0 \mathrm{~mL})$. To the solution was added $\mathrm{KHCO}_{3}(0.42 \mathrm{~g}, 4.2 \mathrm{mmol})$ followed by slow addition of $30 \% \mathrm{H}_{2} \mathrm{O}_{2}(1.9 \mathrm{~mL}, 17 \mathrm{mmol})$ at r.t. The reaction mixture was stirred at r.t. overnight. The reaction was quenched by the careful dropwise addition of an satd aq sodium thiosulfate solution $(10 \mathrm{~mL})$ and the solution was then diluted with satd aq $\mathrm{NaHCO}_{3}$ solution $(10 \mathrm{~mL})$ and finally extracted with EtOAc. The organic phase was washed with brine, dried over $\mathrm{Na}_{2} \mathrm{SO}_{4}$, filtered and concentrated under $40{ }^{\circ} \mathrm{C}$ for 1 $\mathrm{h}$ to ensure all of the ester $\mathbf{3 6}$ were converted to lactone. The resulting residue was purified by chromatography (gradient $10 \rightarrow 12 \%$ EtOAc in hexane) to afford $(\boldsymbol{S})-9(0.11$ $\mathrm{g}, 40 \%$ yield $)$ and $(\boldsymbol{R})-\mathbf{9}(0.097 \mathrm{~g}, 35 \%$ yield $)$ as colorless oils. Data for $(\boldsymbol{S})-\mathbf{9}: R_{\mathrm{f}} 0.26$ $\left(5: 2\right.$ hexane-EtOAc); $[\alpha]_{\mathrm{D}}=+8.1\left(c 0.2, \mathrm{CH}_{2} \mathrm{Cl}_{2}\right) ;{ }^{1} \mathrm{H} \mathrm{NMR}\left(600 \mathrm{MHz}, \mathrm{CDCl}_{3}, \delta_{\mathrm{H}}\right) 4.50$ (dddd, $1 \mathrm{H}, J=14.5,8.0,5.5,3.0 \mathrm{~Hz}$ ), 3.87-3.83 (m, $1 \mathrm{H}), 2.71$ (ddd, $1 \mathrm{H}, J=17.5$, 6.0, $1.8 \mathrm{~Hz}$ ), 2.08 (dd, $1 \mathrm{H}, J=17.5,10.5 \mathrm{~Hz}$ ), 2.04 (dddd, $1 \mathrm{H}, J=14.0,4.5,3.0,2.0$ Hz), 2.00 (br s, $1 \mathrm{H}), 1.97-1.92$ (m, $1 \mathrm{H}), 1.88$ (ddd, $1 \mathrm{H}, J=14.5,9.0,8.0 \mathrm{~Hz}), 1.74$ (ddd, $1 \mathrm{H}, J=14.5,5.5,3.5 \mathrm{~Hz}), 1.51-1.27(\mathrm{~m}, 21 \mathrm{H}), 0.90(\mathrm{t}, 3 \mathrm{H}, J=7.2 \mathrm{~Hz}), 0.90$ $(\mathrm{t}, 3 \mathrm{H}, J=7.2 \mathrm{~Hz}) ;{ }^{13} \mathrm{C} \mathrm{NMR}\left(150 \mathrm{MHz}, \mathrm{CDCl}_{3}, \delta_{\mathrm{C}}\right)$ 171.1, 79.6, 69.4, 43.3, 37.7, $36.40,36.39,35.3,31.8,31.7,31.6,29.3,29.2,26.3,25.4,22.6,14.07,14.05 ;$ HRMS (ESI) calcd for $(\mathrm{M}+\mathrm{Na}) \mathrm{C}_{19} \mathrm{H}_{36} \mathrm{NaO}_{3}: 335.2562$. Found: 335.2553. Data for $(\boldsymbol{R})-\mathbf{9}: R_{\mathrm{f}}$ $0.27\left(5: 2\right.$ hexane-EtOAc); $[\alpha]_{\mathrm{D}}=+1.9\left(c 0.2, \mathrm{CH}_{2} \mathrm{Cl}_{2}\right) ;{ }^{1} \mathrm{H} \mathrm{NMR}\left(600 \mathrm{MHz}, \mathrm{CDCl}_{3}, \delta_{\mathrm{H}}\right)$ 
4.68 (app tdd, $1 \mathrm{H}, J=10.0,4.0,3.0 \mathrm{~Hz}$ ), 3.99-3.96 (m, $1 \mathrm{H}), 2.58$ (dd, $1 \mathrm{H}, J=16.0$, $6.0 \mathrm{~Hz}), 2.22(\mathrm{dd}, 1 \mathrm{H}, J=16.0,9.5 \mathrm{~Hz}), 2.06-2.00(\mathrm{~m}, 1 \mathrm{H}), 1.83-1.77(\mathrm{~m}, 2 \mathrm{H}), 1.71$ (br s, $1 \mathrm{H}), 1.64$ (ddd, $1 \mathrm{H}, J=14.0,5.5,4.0 \mathrm{~Hz}), 1.56$ (ddd, $1 \mathrm{H}, J=14.5,10.0,2.7$ $\mathrm{Hz}), 1.49-1.28(\mathrm{~m}, 20 \mathrm{H}), 0.902(\mathrm{t}, 3 \mathrm{H}, J=7.2 \mathrm{~Hz}), 0.901(\mathrm{t}, 3 \mathrm{H}, J=7.2 \mathrm{~Hz}) ;{ }^{13} \mathrm{C}$ NMR $\left(150 \mathrm{MHz}, \mathrm{CDCl}_{3}, \delta_{\mathrm{C}}\right)$ 172.9, 74.1, 67.6, 43.0, 38.1, 36.1, 35.8, 34.0, 31.8, 31.7, 29.4, 29.2, 27.1, 26.7, 25.5, 22.6, 14.07, 14.05; HRMS (ESI) m/z [M + Na] ${ }^{+}$Calcd for $\mathrm{C}_{19} \mathrm{H}_{36} \mathrm{NaO}_{3} 335.2562$; Found 335.2550.

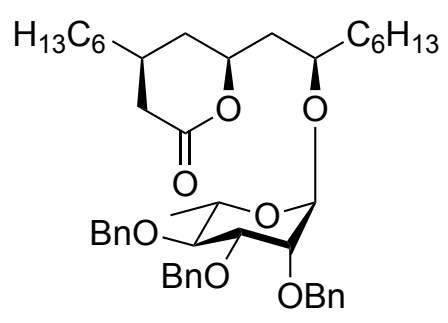

$(4 S, 6 S)-4-h e x y l-6-((R)-2,3,4-t r i-O$-benzyl- $\alpha$-L-rhamnopyranosyl)tetrahydro-2Hpyran-2-one (37): A mixture of acceptor $(S)-9 \quad(0.10 \mathrm{~g}, 0.30 \mathrm{mmol})$, trichloroacetimidate donor $5(0.31 \mathrm{~g}, 0.54 \mathrm{mmol})$ and powdered $4 \AA$ molecular sieves was suspended in anhydrous $\mathrm{Et}_{2} \mathrm{O}(8.0 \mathrm{~mL})$ and stirred at for $10 \mathrm{~min}$. The solution was cooled to $0{ }^{\circ} \mathrm{C}$, and then TMSOTf $(10 \mu \mathrm{L})$ was added. The solution was stirred for $1 \mathrm{~h}$ and then $\mathrm{Et}_{3} \mathrm{~N}(0.50 \mathrm{~mL})$ was added and the mixture was filtered. The filtrate was concentrated and the resulting residue was purified by chromatography (gradient $5 \rightarrow 16 \%$ EtOAc in hexane $)$ to afford $37(0.15 \mathrm{~g}, 63 \%$ yield $)$ as a colorless oil; $R_{\mathrm{f}} 0.31$ (5:1 hexane-EtOAc); $[\alpha]_{\mathrm{D}}=-19.3\left(c \quad 0.2, \mathrm{CH}_{2} \mathrm{Cl}_{2}\right) ;{ }^{1} \mathrm{H}$ NMR $\left(700 \mathrm{MHz}, \mathrm{CDCl}_{3}, \delta_{\mathrm{H}}\right)$ 7.36-7.25 (m, $15 \mathrm{H}), 4.92(\mathrm{~d}, 1 \mathrm{H}, J=11.0 \mathrm{~Hz}), 4.77$ (d, $1 \mathrm{H}, J=12.0 \mathrm{~Hz}), 4.75(\mathrm{~d}, 1$ $\mathrm{H}, J=1.5 \mathrm{~Hz}), 4.67(\mathrm{~d}, 1 \mathrm{H}, J=12.0 \mathrm{~Hz}), 4.64-4.61(\mathrm{~m}, 3 \mathrm{H}), 4.36-4.32(\mathrm{~m}, 1 \mathrm{H})$, 
$3.78(\mathrm{dd}, 1 \mathrm{H}, J=9.0,3.0 \mathrm{~Hz}), 3.71-3.66(\mathrm{~m}, 2 \mathrm{H}), 3.62-3.58(\mathrm{~m}, 2 \mathrm{H}), 2.66(\mathrm{ddd}, 1$ $\mathrm{H}, J=17.5,6.0,1.5 \mathrm{~Hz}), 2.05-1.97(\mathrm{~m}, 3 \mathrm{H}), 1.88-1.84(\mathrm{~m}, 1 \mathrm{H}), 1.61$ (ddd, $1 \mathrm{H}, J=$ 14.0, 7.0, 5.0 Hz), 1.42-1.20 (m, $24 \mathrm{H}), 0.89$ (t, $3 \mathrm{H}, J=7.2 \mathrm{~Hz}), 0.87$ (t, $3 \mathrm{H}, J=7.2$ $\mathrm{Hz}) ;{ }^{13} \mathrm{C} \mathrm{NMR}\left(125 \mathrm{MHz}, \mathrm{CDCl}_{3}, \delta_{\mathrm{C}}\right) 171.3,138.6,138.5,138.2,128.4,128.38,128.37$, $128.1,128.0,127.8,127.7,127.67,127.61,97.2,80.4,79.9,77.7,75.4,75.1,74.2,72.9$, $72.3,68.7,40.9,36.5,36.4,35.1,33.3,31.8,31.7,31.6,29.4,29.2,26.3,24.7,22.7$, 22.6, 18.1, 14.13, 14.07; gHSQC 2-D NMR (without ${ }^{1} \mathrm{H}$ decoupling): ${ }^{1} J_{\mathrm{C} 1, \mathrm{H} 1}=168.4 \mathrm{~Hz}$. HRMS (ESI) m/z [M + Na $]^{+}$Calcd for $\mathrm{C}_{46} \mathrm{H}_{64} \mathrm{NaO}_{7} 751.4544$; Found 751.4544.

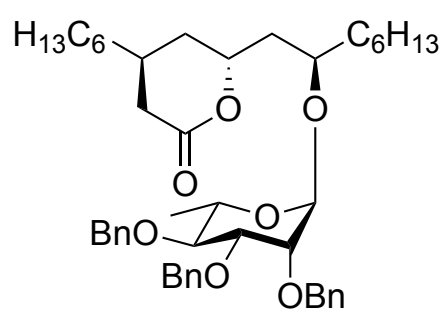

(4S,6R)-4-hexyl-6-((R)-2,3,4-tri- $O$-benzyl- $\alpha$-L-rhamnopyranosyl)tetrahydro-2Hpyran-2-one (38): A mixture of acceptor $(\boldsymbol{R})-9 \quad(60 \mathrm{mg}, 0.18 \mathrm{mmol})$, trichloroacetimidate donor $5(0.19 \mathrm{~g}, 0.32 \mathrm{mmol})$ and powdered $4 \AA$ molecular sieves was suspended in anhydrous $\mathrm{Et}_{2} \mathrm{O}(8 \mathrm{~mL})$ and stirred for $10 \mathrm{~min}$. The solution was then cooled to $0{ }^{\circ} \mathrm{C}$ and TMSOTf $(10 \mu \mathrm{L})$ was added. The solution was stirred for $1 \mathrm{~h}$ before $\mathrm{Et}_{3} \mathrm{~N}(0.50 \mathrm{~mL})$ was added and the mixture was filtered. The filtrate was concentrated and the resulting residue was purified by chromatography (gradient $5 \rightarrow 16 \%$ EtOAc in hexane) to afford $\mathbf{3 8}(90 \mathrm{mg}, 64 \%$ yield $)$ as a colorless oil; $R_{\mathrm{f}} 0.30$ (5:1 hexane-EtOAc); $[\alpha]_{\mathrm{D}}=-16.2\left(c 0.3, \mathrm{CH}_{2} \mathrm{Cl}_{2}\right) ;{ }^{1} \mathrm{H} \mathrm{NMR}\left(600 \mathrm{MHz}, \mathrm{CDCl}_{3}, \delta_{\mathrm{H}}\right) 7.39-7.29(\mathrm{~m}, 15 \mathrm{H})$, $4.94(\mathrm{~d}, 1 \mathrm{H}, J=11.0 \mathrm{~Hz}), 4.82(\mathrm{~d}, 1 \mathrm{H}, J=2.0 \mathrm{~Hz}), 4.79(\mathrm{~d}, 1 \mathrm{H}, J=12.0 \mathrm{~Hz}), 4.72$ 
(d, $1 \mathrm{H}, J=12.0 \mathrm{~Hz}), 4.66(\mathrm{~d}, 1 \mathrm{H}, J=11.0 \mathrm{~Hz}), 4.64(\mathrm{~s}, 2 \mathrm{H}), 4.53-4.49(\mathrm{~m}, 1 \mathrm{H})$, 3.89-3.85 (m, 1H), $3.80(\mathrm{dd}, 1 \mathrm{H}, J=9.0,3.0 \mathrm{~Hz}), 3.70(\mathrm{dd}, 1 \mathrm{H}, J=3.0,2.0 \mathrm{~Hz})$, 3.68-3.61 (m, $2 \mathrm{H}), 2.53(\mathrm{dd}, 1 \mathrm{H}, J=16.0,6.0 \mathrm{~Hz}), 2.14(\mathrm{dd}, 1 \mathrm{H}, J=16.0,10.0 \mathrm{~Hz})$, 2.01-1.96 (m, $1 \mathrm{H}), 1.76-1.70(\mathrm{~m}, 1 \mathrm{H}), 1.59-1.53$ (m, $1 \mathrm{H}), 1.38-1.23$ (m, $24 \mathrm{H}), 0.92$ (t, $3 \mathrm{H}, J=7.2 \mathrm{~Hz}$ ), 0.89 (t, $3 \mathrm{H}, J=7.2 \mathrm{~Hz}$ ); HRMS (ESI) $\mathrm{m} / \mathrm{z}[\mathrm{M}+\mathrm{Na}]^{+}$Calcd for $\mathrm{C}_{46} \mathrm{H}_{64} \mathrm{NaO}_{7} 751.4544$; Found 751.4539 .

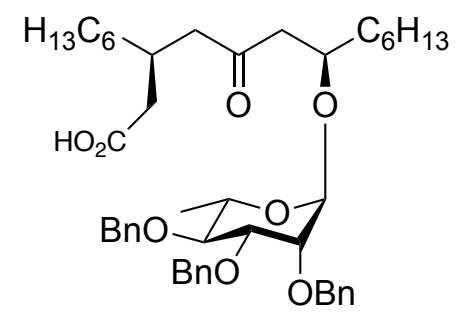

(3S,7R)-3-hexyl-7-(2,3,4-tri- $O$-benzyl- $\alpha$-L-rhamnopyranosyl)-5-oxo-tridecanoic acid (39): A solution of 37 (70 mg, $0.096 \mathrm{mmol})$ in $\mathrm{CH}_{3} \mathrm{OH}$-water $(4: 1,25 \mathrm{~mL})$ was treated with $\mathrm{LiOH} \cdot \mathrm{H}_{2} \mathrm{O}(40 \mathrm{mg}, 0.96 \mathrm{mmol})$ and was stirred overnight. The reaction mixture was acidified to $\mathrm{pH} 5$ by the addition of $1.0 \mathrm{M} \mathrm{HCl}$, diluted with water and extracted with $\mathrm{CH}_{2} \mathrm{Cl}_{2}$. The organic phase was washed with brine, dried over $\mathrm{Na}_{2} \mathrm{SO}_{4}$, filtere and concentrated to afford the alcohol intermediate in $\mathrm{CH}_{2} \mathrm{Cl}_{2}$, which was used immediately without further purification. To a mixture of the alcohol intermediate and $4 \AA$ molecular sieves in dry $\mathrm{CH}_{2} \mathrm{Cl}_{2}$ were added NMO (22 mg, $\left.0.19 \mathrm{mmol}\right)$ and TPAP (4.0 mg, $0.011 \mathrm{mmol}$ ) at r.t. The mixture was stirred for $6 \mathrm{~h}$ and then concentrated and the crude product was purified by chromatography (gradient $30 \rightarrow 50 \%$ EtOAc-hexane) to afford 39 (51 $\mathrm{mg}, 71 \%$ yield) as a colorless oil. Compound $\mathbf{3 8}(50 \mathrm{mg}, 0.069 \mathrm{mmol})$ was converted to 39 (36 mg, 70\% yield) using same method. $[\alpha]_{\mathrm{D}}=-25.6(c \quad 0.4$, 
$\left.\mathrm{CH}_{2} \mathrm{Cl}_{2}\right) ;{ }^{1} \mathrm{H} \mathrm{NMR}\left(600 \mathrm{MHz}, \mathrm{CD}_{3} \mathrm{OD}, \delta_{\mathrm{H}}\right) 7.37-7.24(\mathrm{~m}, 15 \mathrm{H}), 4.85(\mathrm{~d}, 1 \mathrm{H}, J=11.0$ Hz), $4.77(\mathrm{~d}, 1 \mathrm{H}, J=1.5 \mathrm{~Hz}), 4.69(\mathrm{~d}, 1 \mathrm{H}, J=12.0 \mathrm{~Hz}), 4.62(\mathrm{~d}, 2 \mathrm{H}, J=12.0 \mathrm{~Hz})$, $4.57(\mathrm{~d}, 1 \mathrm{H}, J=12.0 \mathrm{~Hz}), 4.55(\mathrm{~d}, 1 \mathrm{H}, J=12.0 \mathrm{~Hz}), 4.07-4.03(\mathrm{~m}, 1 \mathrm{H}), 3.73(\mathrm{dd}, 1$ $\mathrm{H}, J=9.5,3.0 \mathrm{~Hz}), 3.68(\mathrm{dd}, 1 \mathrm{H}, J=3.0,1.5 \mathrm{~Hz}), 3.64-3.59(\mathrm{~m}, 1 \mathrm{H}), 3.50$ (app t, 1 $\mathrm{H}, J=9.5 \mathrm{~Hz}), 2.68(\mathrm{dd}, 1 \mathrm{H}, J=16.0,7.5 \mathrm{~Hz}), 2.50(\mathrm{dd}, 1 \mathrm{H}, J=16.0,5.0 \mathrm{~Hz}), 2.48$ $(\mathrm{d}, 2 \mathrm{H}, J=6.5 \mathrm{~Hz}), 2.32-2.28(\mathrm{~m}, 3 \mathrm{H}), 2.238(\mathrm{~d}, 1 \mathrm{H}, J=6.5 \mathrm{~Hz}), 2.237(\mathrm{~d}, 1 \mathrm{H}, J=$ $7.0 \mathrm{~Hz}), 1.41-1.20(\mathrm{~m}, 23 \mathrm{H}), 0.91(\mathrm{t}, 3 \mathrm{H}, J=7.2 \mathrm{~Hz}), 0.86(\mathrm{t}, 3 \mathrm{H}, J=7.2 \mathrm{~Hz}) ;{ }^{13} \mathrm{C}$ NMR (150 MHz, $\left.\mathrm{CD}_{3} \mathrm{OD}, \delta_{\mathrm{C}}\right)$ 209.1, 175.3, 138.6, 138.4, 138.1, 128.1, 128.01, 127.98, $127.8,127.68,127.66,127.5,127.3,127.2,96.3,80.0,79.4,75.1,74.5,73.5,72.4,71.7$, $68.2,38.0,33.7,32.9,31.53,31.50,30.6,29.1,29.0,26.3,24.4,22.28,22.24,16.9$, 13.04, 12.99; HRMS (ESI) $\mathrm{m} / \mathrm{z}[\mathrm{M}-\mathrm{H}]^{-}$Calcd for $\mathrm{C}_{46} \mathrm{H}_{63} \mathrm{O}_{8}$ 743.4528; Found 743.4533.

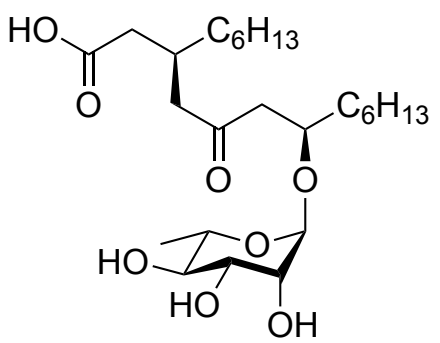

(3S,7R)-3-hexyl-7-( $\alpha$-L-rhamnopyranosyl)-5-oxo-tridecanoic acid (4): To a solution of $39(65 \mathrm{mg}, 0.087 \mathrm{mmol})$ in $\mathrm{CH}_{3} \mathrm{OH}(10 \mathrm{~mL})$, and acetic acid $(0.20 \mathrm{~mL})$ was treated with palladium on charcoal $(10 \%, 50 \mathrm{mg})$ and subjected to a hydrogen atmosphere for $20 \mathrm{~h}$. The mixture was filtered through Celite and the filtrate was concentrated. The residue was purified by chromatography (Iatrobeads 6RS-8060, gradient $10 \rightarrow 40 \% \mathrm{CH}_{3} \mathrm{OH}-\mathrm{CH}_{2} \mathrm{Cl}_{2}$ ) to give 4 (33 $\mathrm{mg}, 81 \%$ yield) as a colorless foam. 
$[\alpha]_{\mathrm{D}}=-45.1\left(c 0.3, \mathrm{CH}_{2} \mathrm{Cl}_{2}\right) ;{ }^{1} \mathrm{H}$ NMR $\left(700 \mathrm{MHz}, \mathrm{CD}_{3} \mathrm{OD}, \delta_{\mathrm{H}}\right) 4.76(\mathrm{~d}, 1 \mathrm{H}, J=1.5$ Hz, H-1), 4.14-4.11 (m, 1 H, $\mathrm{OCHCH}_{2}$ ), 3.72 (dd, $\left.1 \mathrm{H}, J=3.5,1.5 \mathrm{~Hz}, \mathrm{H}-2\right), 3.57$ (dd, $1 \mathrm{H}, J=9.5,3.5 \mathrm{~Hz}, \mathrm{H}-3), 3.55(\mathrm{dq}, 1 \mathrm{H}, J=9.5,6.0 \mathrm{~Hz}, \mathrm{H}-5), 3.34$ (app t, $1 \mathrm{H}, J=$ $9.5 \mathrm{~Hz}, \mathrm{H}-4), 2.75$ (dd, $1 \mathrm{H}, J=16.0,7.5 \mathrm{~Hz}, \mathrm{CH}_{2} \mathrm{CHCH}_{2} \mathrm{CO}_{2} \mathrm{H}$ ), 2.56 (dd, $1 \mathrm{H}, J=$ 16.0, $\left.5.0 \mathrm{~Hz}, \mathrm{OCHCH}_{2} \mathrm{C}=\mathrm{O}\right), 2.54\left(\mathrm{dd}, 1 \mathrm{H}, J=17.0,7.0 \mathrm{~Hz}, \mathrm{CH}_{2} \mathrm{CHCH}_{2} \mathrm{CO}_{2} \mathrm{H}\right), 2.51$ (dd, $\left.1 \mathrm{H}, J=17.0,6.5 \mathrm{~Hz}, \mathrm{CH}_{2} \mathrm{CHCH}_{2} \mathrm{CO}_{2} \mathrm{H}\right), 2.35-2.32\left(\mathrm{~m}, 1 \mathrm{H}, \mathrm{CHCH}_{2} \mathrm{CO}_{2} \mathrm{H}\right), 2.29$ $\left(\mathrm{dd}, 1 \mathrm{H}, J=15.0,6.0 \mathrm{~Hz}, \mathrm{CH}_{2} \mathrm{CO}_{2} \mathrm{H}\right), 2.24\left(\mathrm{dd}, 1 \mathrm{H}, J=15.0,7.0 \mathrm{~Hz}, \mathrm{CH}_{2} \mathrm{CO}_{2} \mathrm{H}\right.$ ), 1.51-1.26 (m, $\left.20 \mathrm{H}, 10 \times \mathrm{CH}_{2}\right), 1.23(\mathrm{~d}, 3 \mathrm{H}, J=6.0 \mathrm{~Hz}, \mathrm{H}-6), 0.90$ (t, $3 \mathrm{H}, J=7.0 \mathrm{~Hz}$, $\left.\mathrm{CH}_{3}\right), 0.89\left(\mathrm{t}, 3 \mathrm{H}, J=7.0 \mathrm{~Hz}, \mathrm{CH}_{3}\right) ;{ }^{13} \mathrm{C} \mathrm{NMR}\left(125 \mathrm{MHz}, \mathrm{CD}_{3} \mathrm{OD}, \delta_{\mathrm{C}}\right) 210.7(\mathrm{C}=\mathrm{O})$, $176.8(\mathrm{C}=\mathrm{OOH}), 100.4(\mathrm{C}-1), 74.9\left(\mathrm{OCHCH}_{2}\right), 74.0(\mathrm{C}-4), 72.7$ (C-2), 72.4 (C-3), 70.3 (C-5), $49.2\left(\mathrm{CH}_{2} \mathrm{C}=\mathrm{O}\right), 49.1\left(\mathrm{CH}_{2} \mathrm{C}=\mathrm{O}\right), 39.4\left(\mathrm{CH}_{2} \mathrm{CO}_{2} \mathrm{H}\right), 35.3\left(\mathrm{CH}_{2}\right), 34.6\left(\mathrm{CH}_{2}\right)$, $32.95\left(\mathrm{CH}_{2}\right), 32.94\left(\mathrm{CH}_{2}\right), 30.51\left(\mathrm{CH}_{2}\right), 30.45\left(\mathrm{CH}_{2}\right), 27.7\left(\mathrm{CH}_{2}\right), 25.9\left(\mathrm{CH}_{2}\right), 23.70$ $\left(\mathrm{CH}_{2}\right), 23.67\left(\mathrm{CH}_{2}\right), 18.0(\mathrm{C}-6), 14.4\left(\mathrm{CH}_{3}\right) ; \mathrm{HRMS}(\mathrm{ESI}) \mathrm{m} / \mathrm{z}[\mathrm{M}+\mathrm{Na}]^{+}$Calcd for $\mathrm{C}_{25} \mathrm{H}_{46} \mathrm{NaO}_{8}$ 497.3085; Found 497.3086.

\section{References:}

(1) Larson, D. P.; Heathcock, C. H. J. Org. Chem. 1997, 62, 8406-8418.

(2) Davis, J. B.; Daniel Bailey, J.; Sello, J. K. Org. Lett. 2009, 11, 2984-2987.

(3) Chintareddy, V. R.; Ellern, A.; Verkade, J. G. J. Org. Chem. 2010, 75, 71667174. 

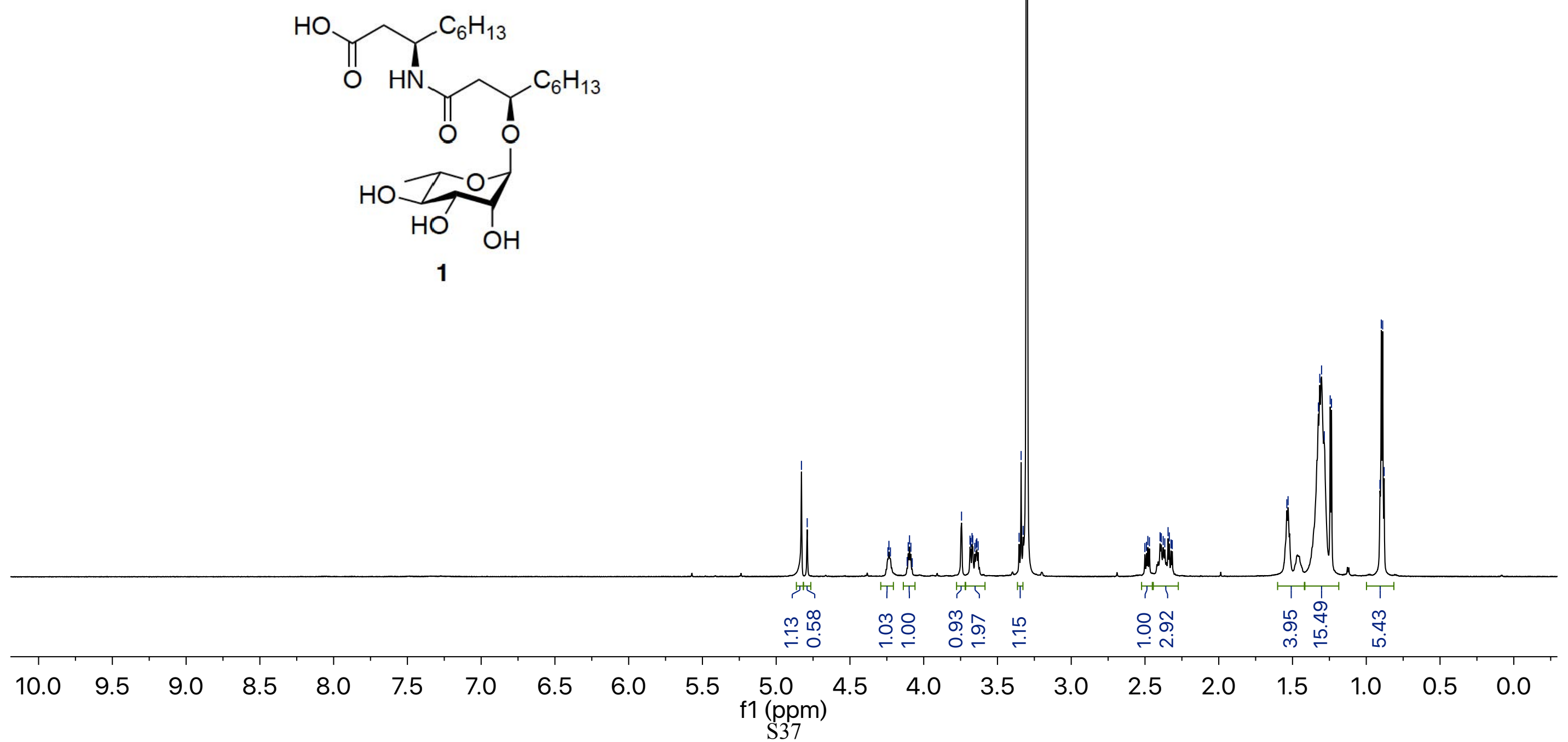
1-C - Lei, WL-1-049-prod - 125.691 MHz C13[H1] DEPTq in cd3od (ref. to CD3OD @ 49.0 ppm), temp 27.7 C -> actual temp = 27.0 C, colddual k 인

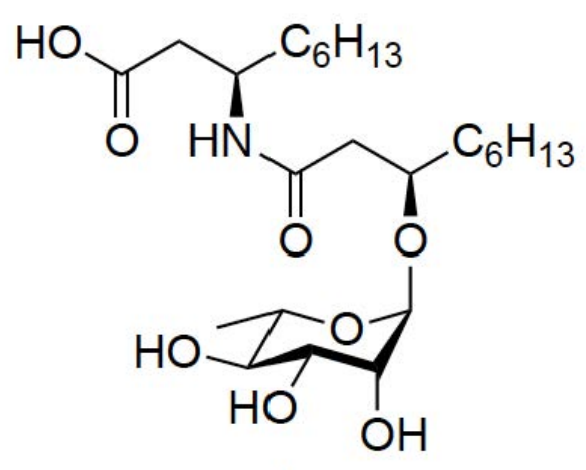

\begin{tabular}{|c|c|c|c|c|c|c|c|c|c|c|c|c|c|c|c|c|c|c|c|}
\hline 200 & 190 & 180 & 170 & 160 & 150 & 140 & 130 & 120 & 110 & $\begin{array}{l}100 \\
\mathrm{f} 1(\mathrm{ppm})\end{array}$ & 80 & 70 & 60 & 50 & 40 & 30 & 20 & 10 & 0 \\
\hline
\end{tabular}


2-H - Lei, WL-1-069-prod - 699.771 MHz H1 PRESAT in cd3od (ref. to CD3OD @ 3.30 ppm), temp $27.5 \mathrm{C}$-> actual temp = 27.0 C, coldid probe -

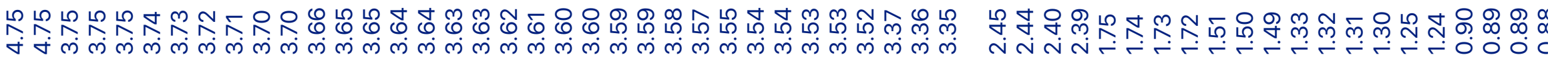

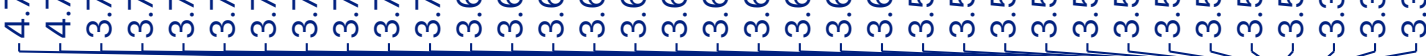

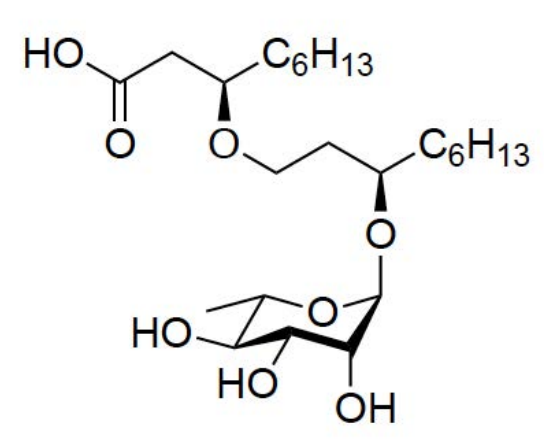

2

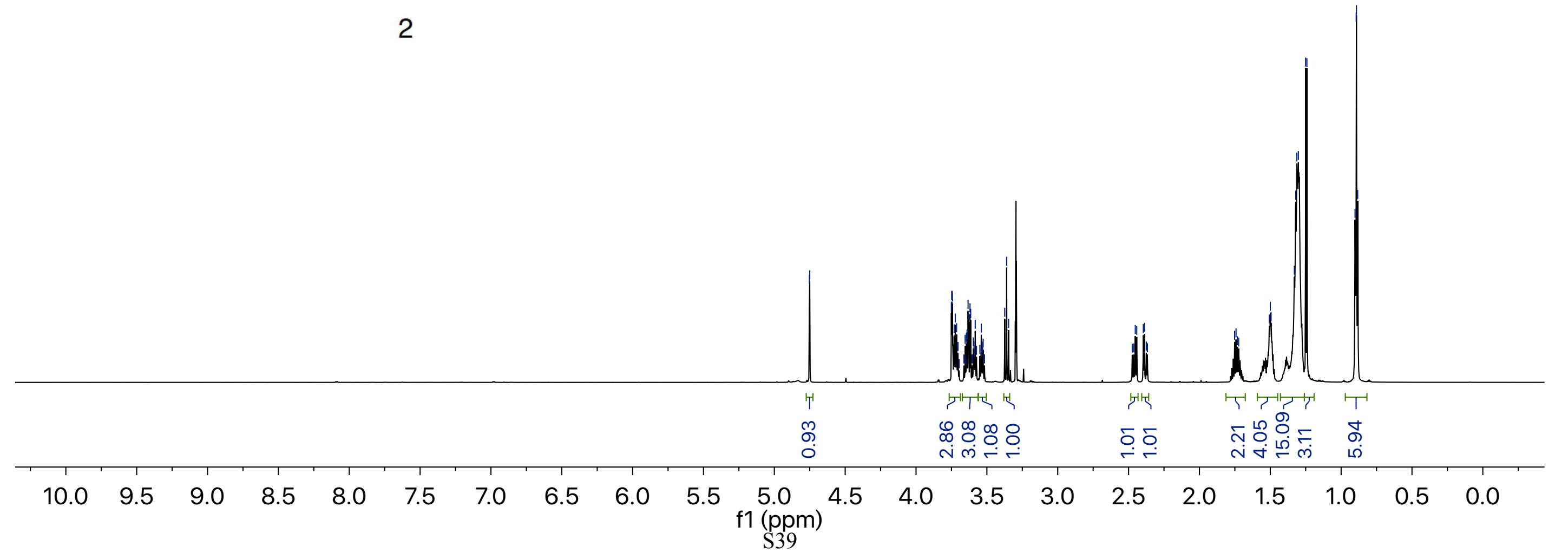


2-C - Lei, WL-1-069-prod - 125.691 MHz C13[H1] DEPTq in cd3od (ref. to CD3OD @ 49.0 ppm), temp 27.7 C -> actual temp = 27.0 C, colddual k 웅

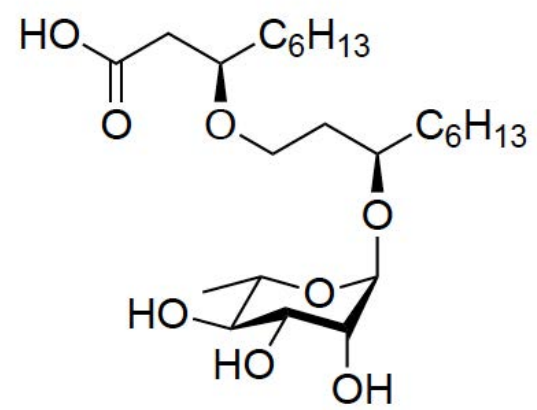

2

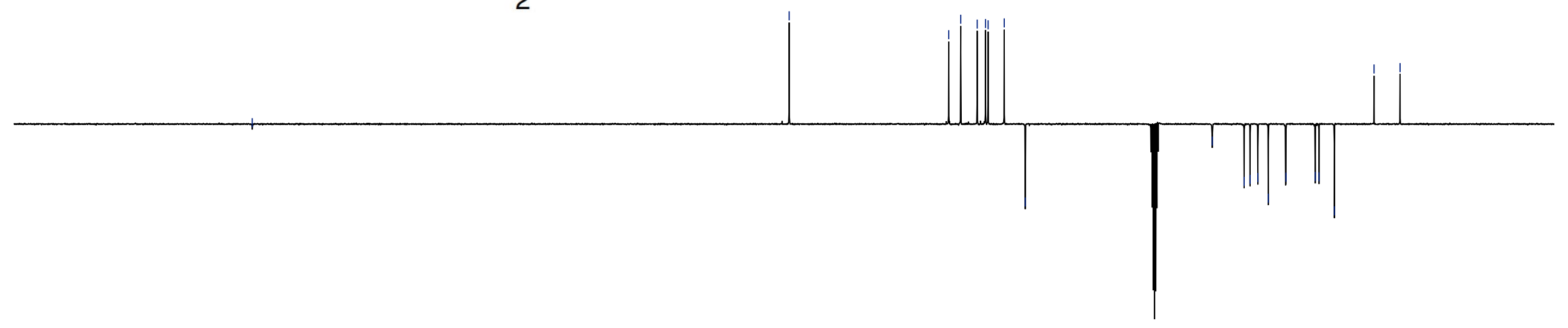






3

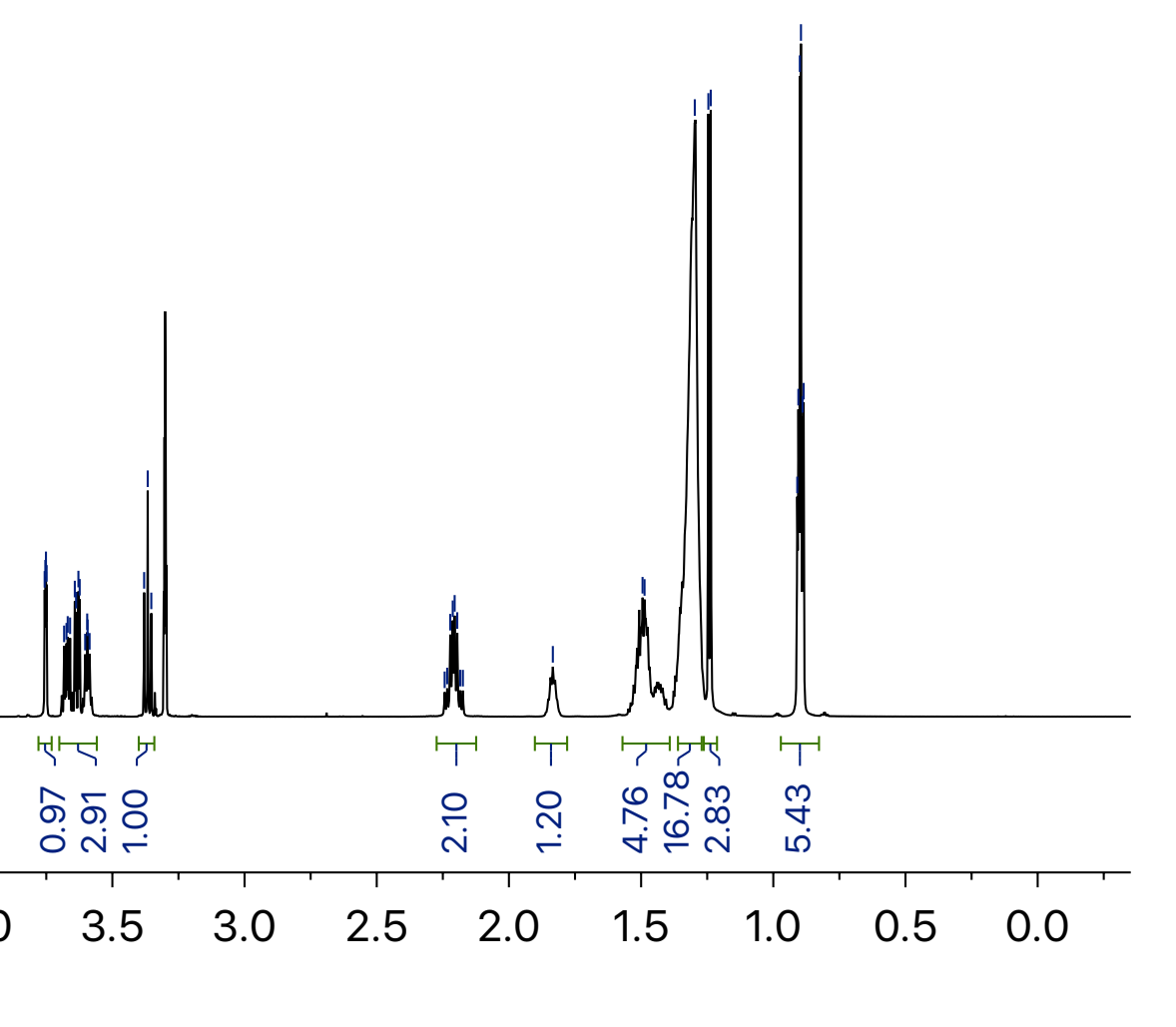


3-C - Lei, WL-2-060-prod - 125.691 MHz C13[H1] DEPTq in cd3od (ref. to CD3OD @ 49.0 ppm), temp 27.7 C -> actual temp = 27.0 C, colddual
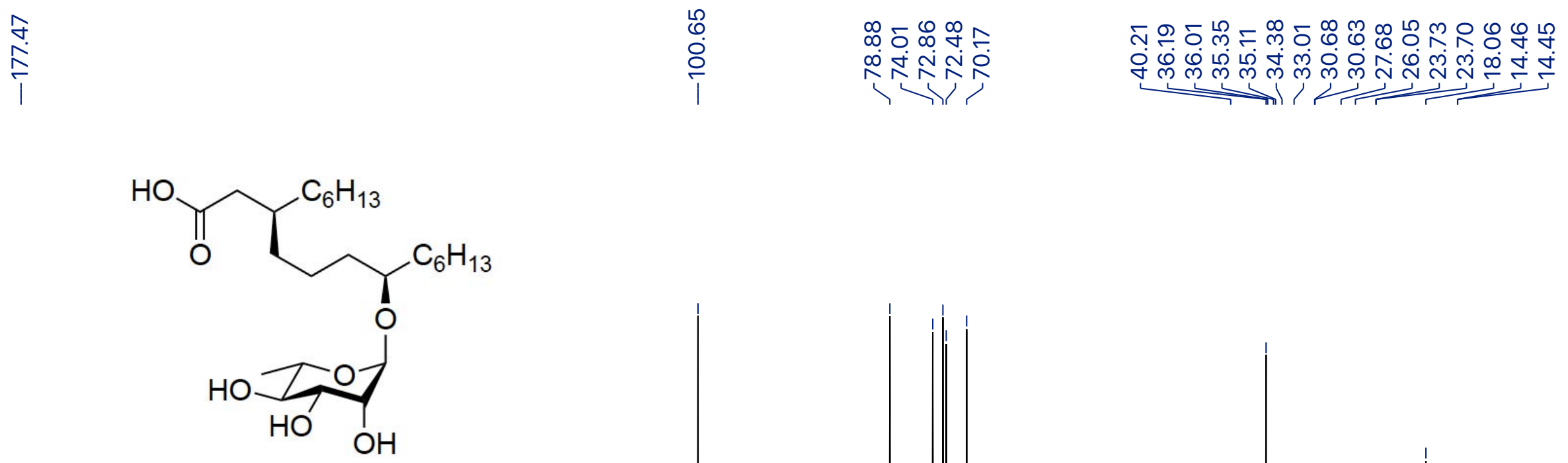

3

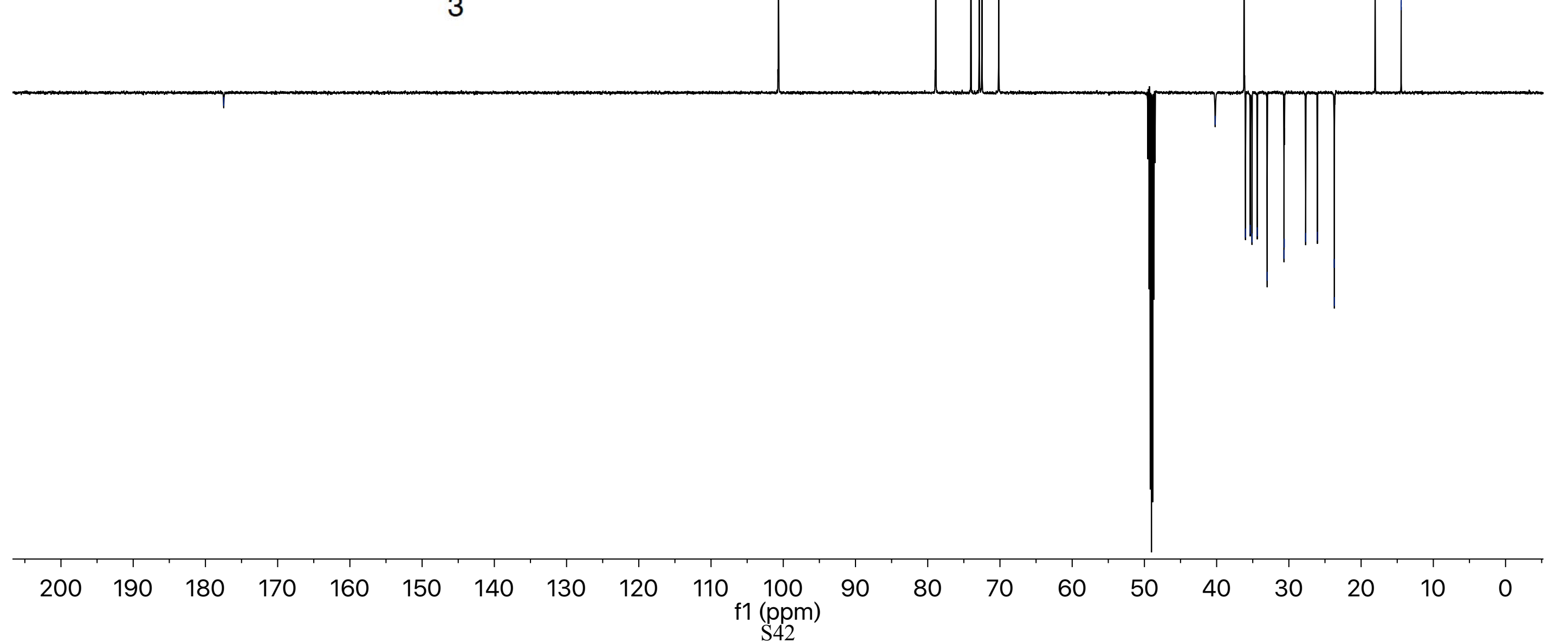




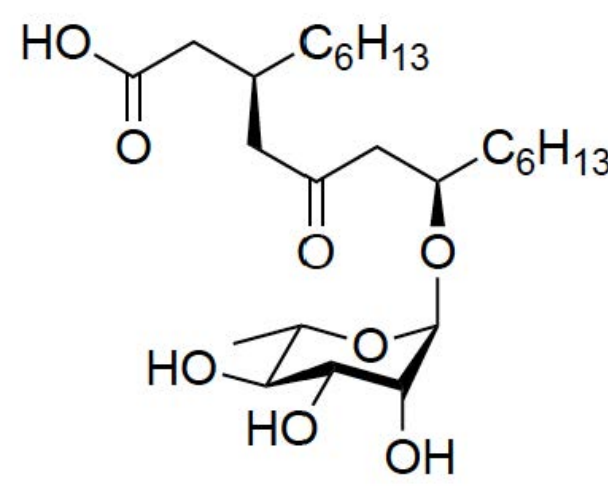

4

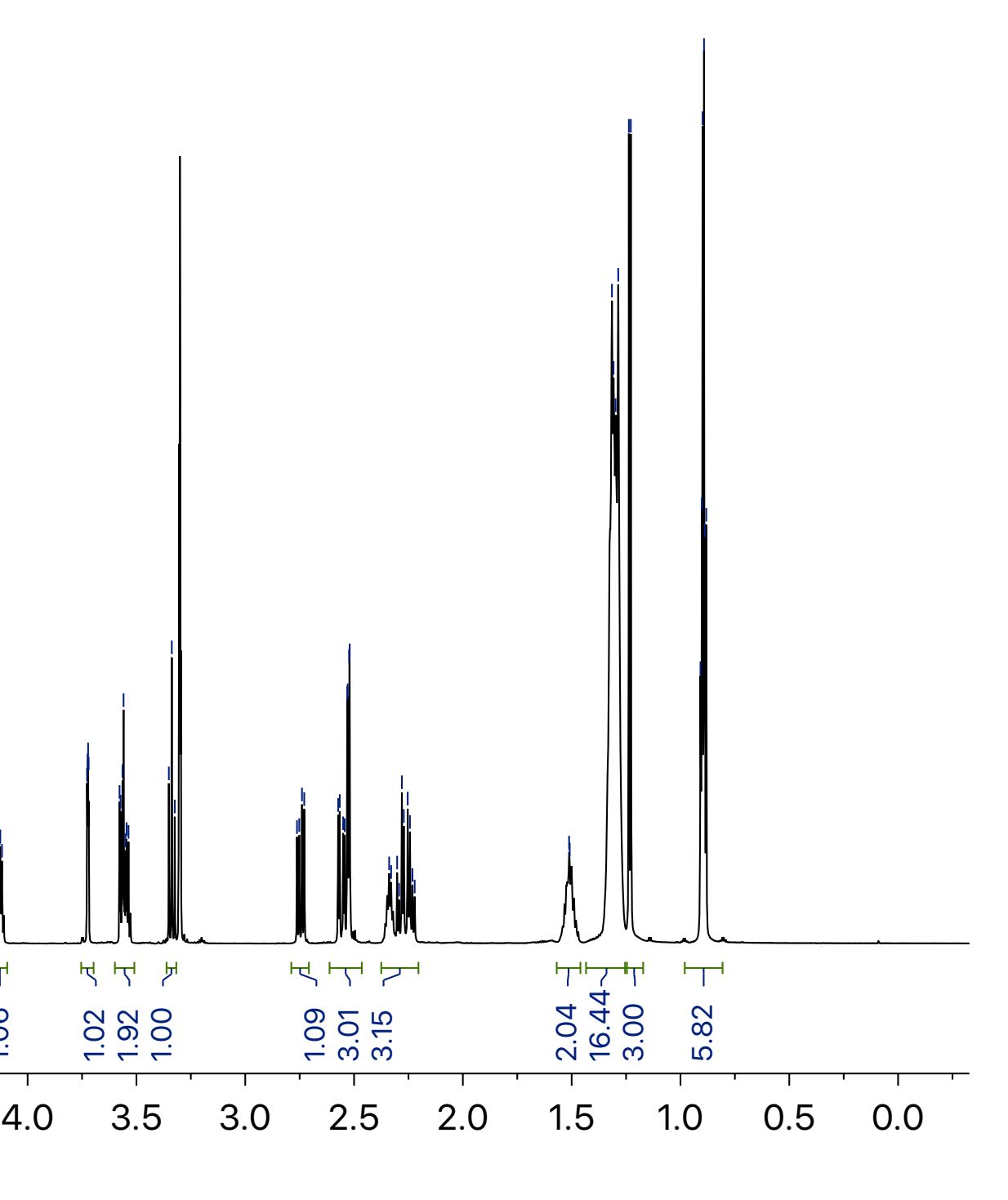


4-C - Lei, WL-2-078-prod — 125.691 MHz C13[H1] DEPTq in cd3od (ref. to CD3OD @ 49.0 ppm), temp 27.7 C -> actual temp = 27.0 C, colddual । \begin{tabular}{l}
$\infty$ \\
0 \\
0 \\
\multirow{1}{1}{}
\end{tabular}

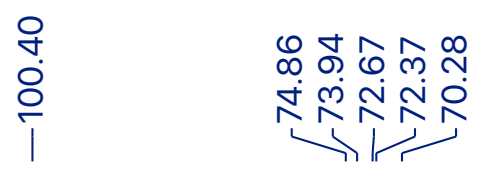



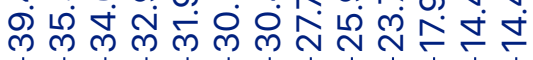

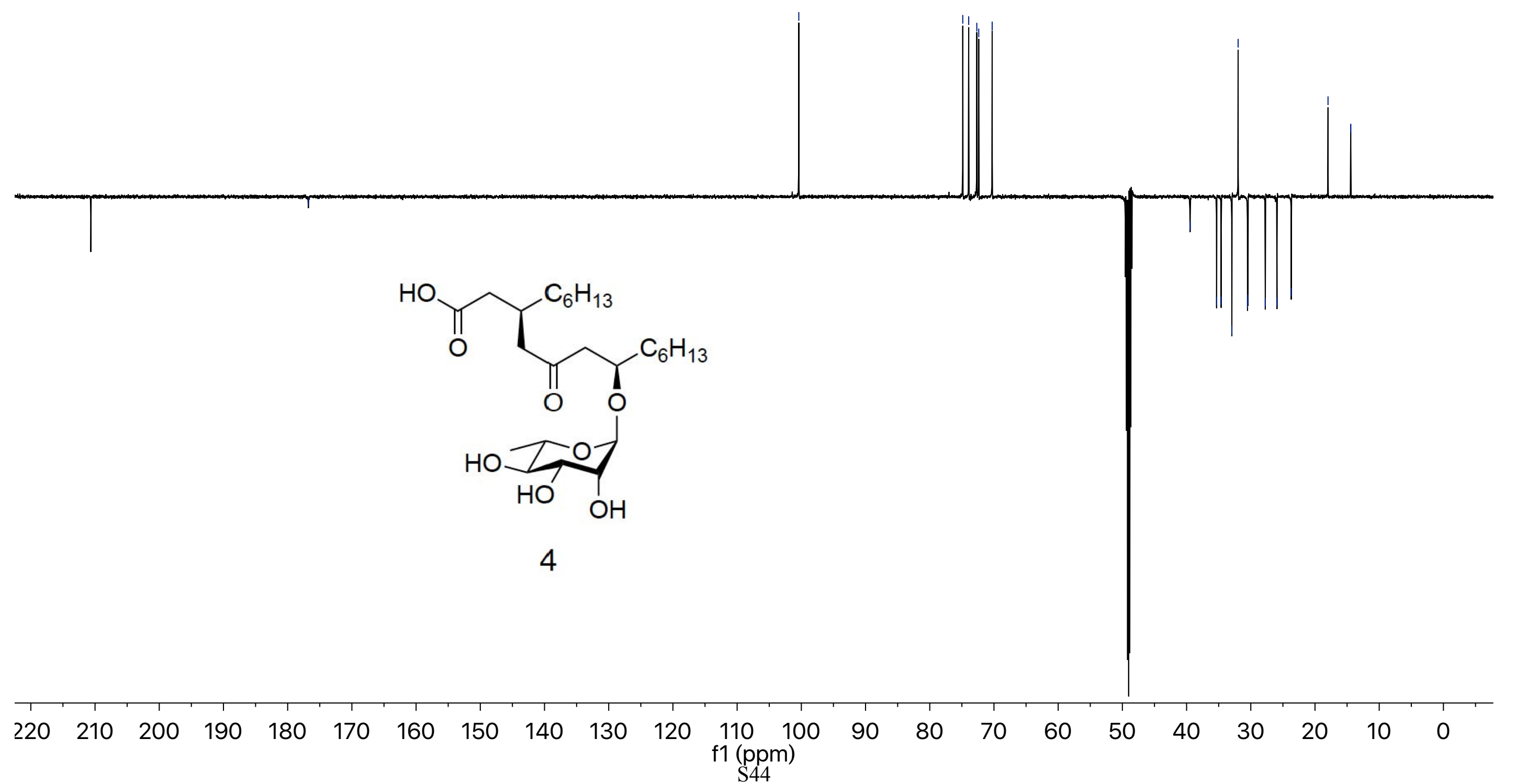




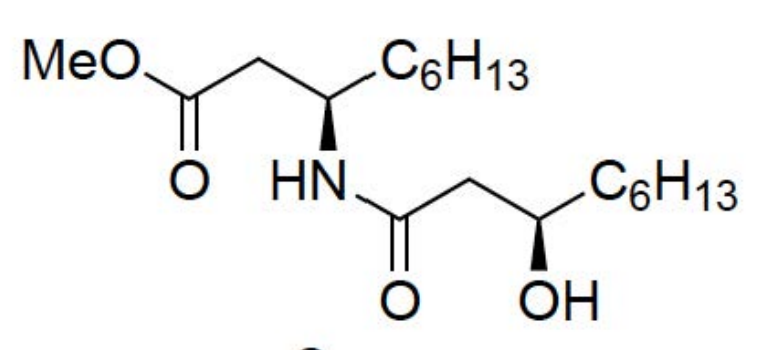

6




6-C - WL-1-035-CNMR - 100.537 MHz C13[H1] 1D in cdcl3 (ref. to CDCl3 @ 77.06 ppm), temp 27.0 C -> actual temp = 27.0 C, autoxdb probe 齐

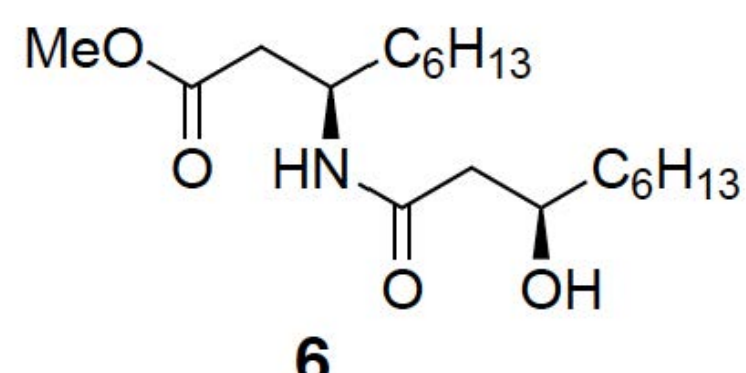

잉 $\quad 0$

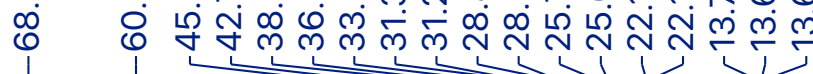

6

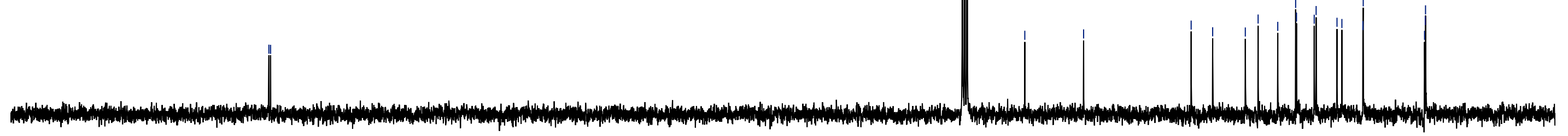


7-H - WL-1-065-product - 599.926 MHz H1 1D in cdcl3 (ref. to CDCl3 @ 7.26 ppm), temp 25.8 C -> actual temp = 27.0 C, autoxid probe -

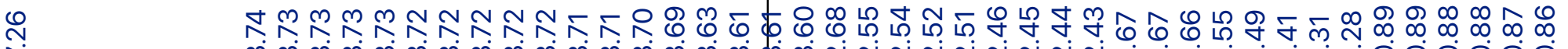

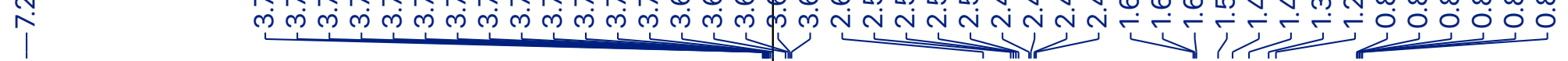

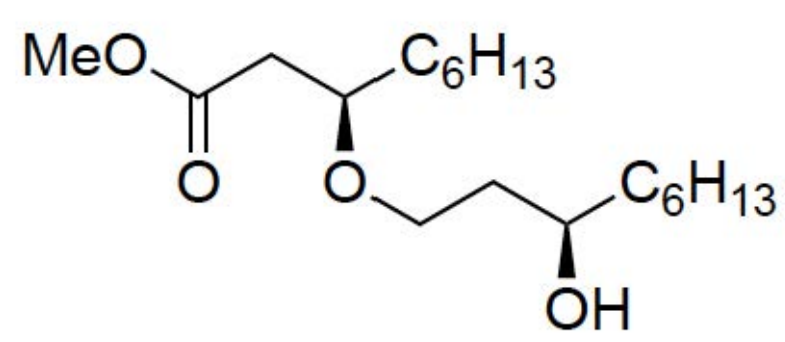

7

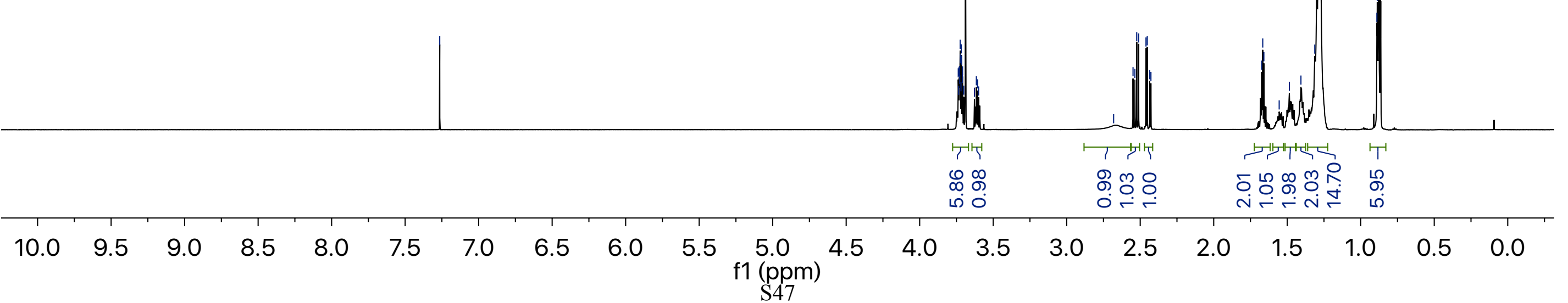


7-C - WL-1-065-CNMR - 150.868 MHz C13[H1] 1D in cdcl3 (ref. to CDCl3 @ 77.06 ppm), temp 25.8 C -> actual temp = 27.0 C, autoxid probe -

$\stackrel{\text { ํ. }}{\text { I }}$
అִ

זั⁄

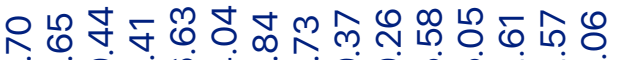
ธ்ุ<smiles>COC(=O)C[C@H](CCCC(O)c1ccccc1)OC</smiles>

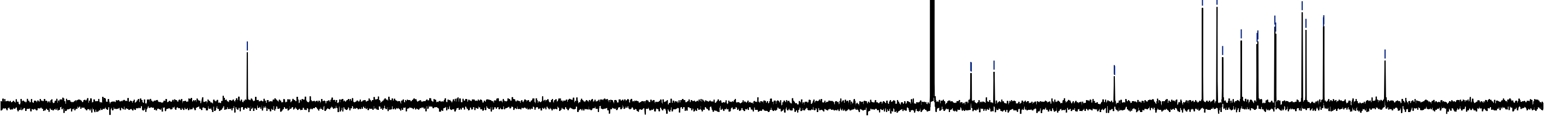

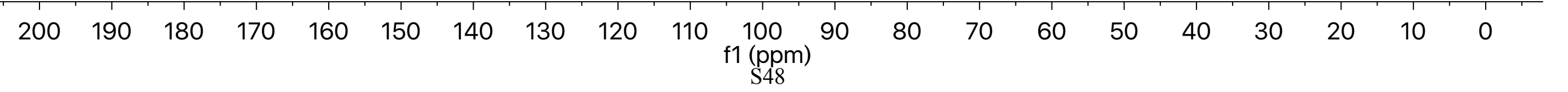


8-H - WL-2-072-prod - 599.926 MHz H1 1D in cdcl3 (ref. to CDCl3 @ 7.26 ppm), temp 25.8 C -> actual temp = 27.0 C, autoxid probe -

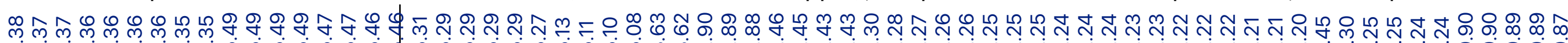

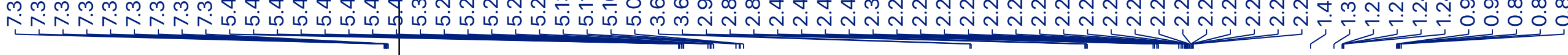

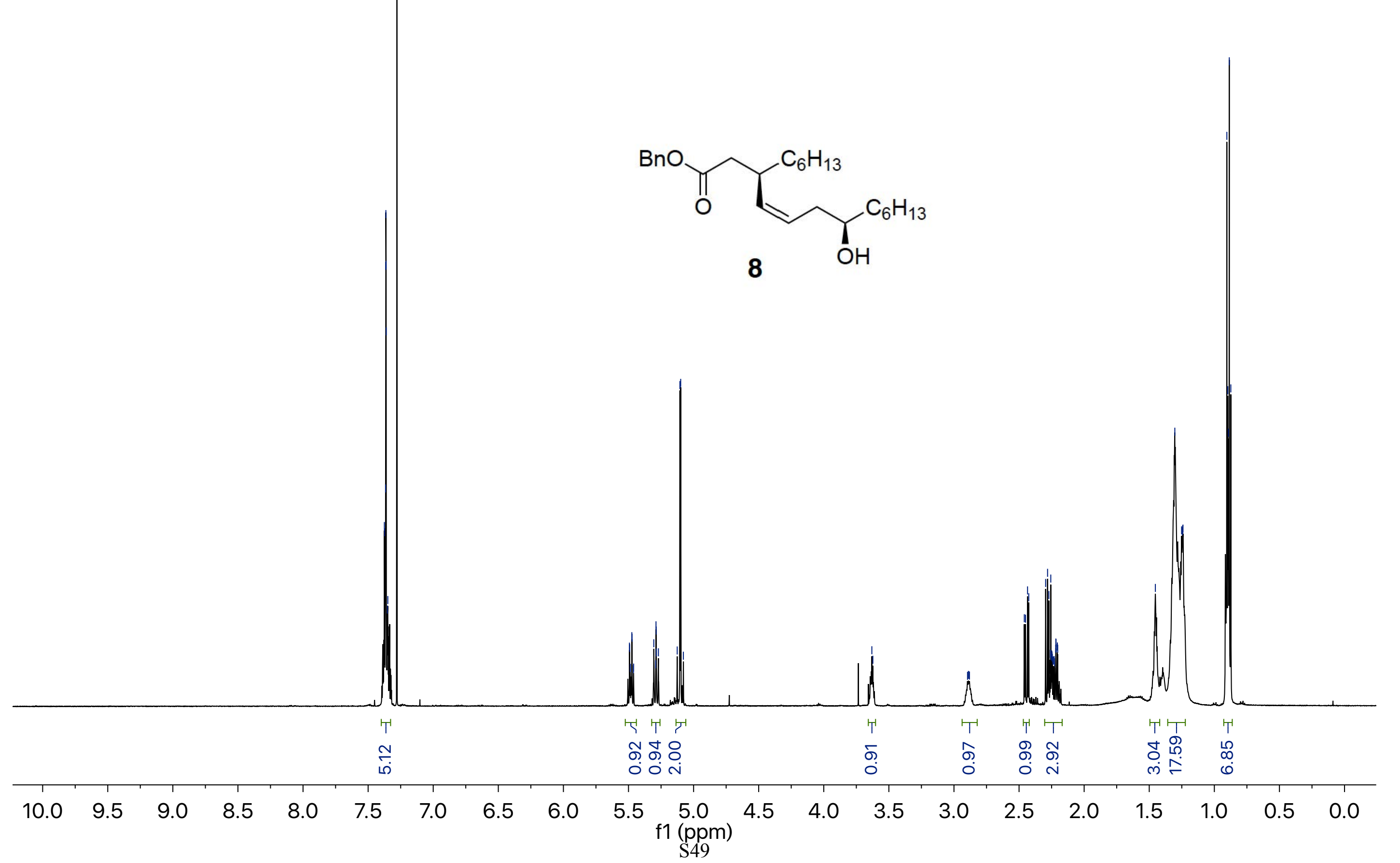


8-C - WL-2-072-prod - 100.586 MHz C13[H1] 1D in cdcl3 (ref. to CDCl3 @ 77.06 ppm), temp 25.9 C -> actual temp = 27.0 C, onenmr probe -

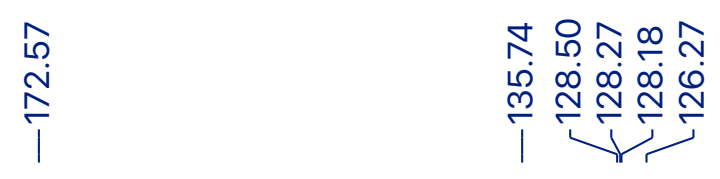

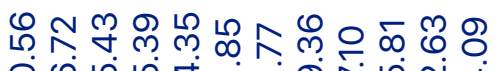

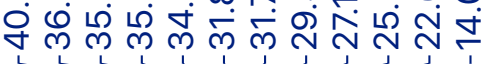

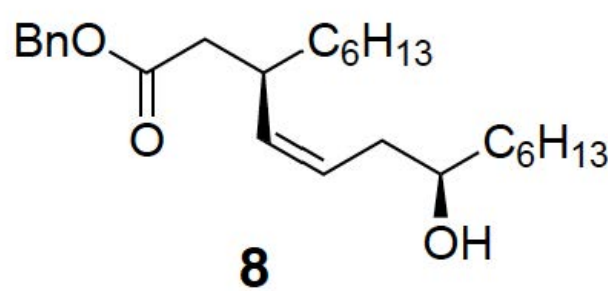

8

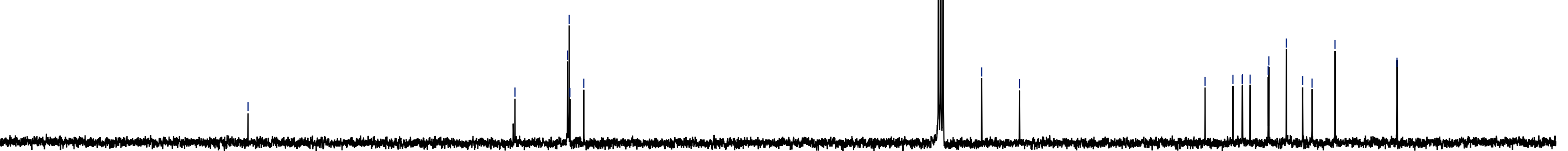


(R)-9-H - WL-2-073-prod-up - 599.926 MHz H1 1D in cdcl3 (ref. to CDCl3 @ 7.26 ppm), temp 25.8 C -> actual temp = 27.0 C, autoxid probe -

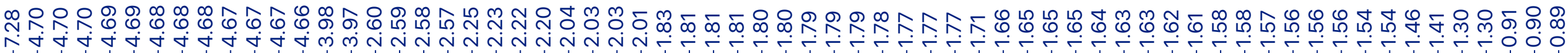

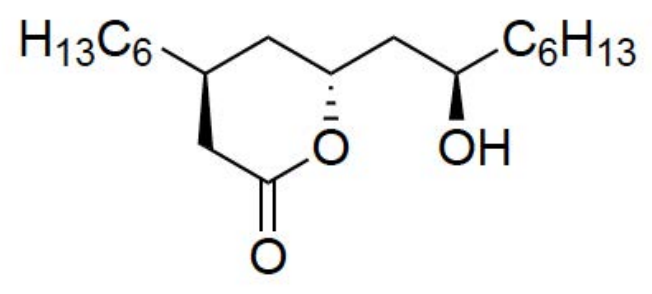

$(R)-9$




(R)-9-C - WL-2-073-up-CNMR - 150.868 MHz C13[H1] 1D in cdcl3 (ref. to CDCl3 @ 77.06 ppm), temp $25.8 \mathrm{C}->$ actual temp = 27.0 C, autoxid k

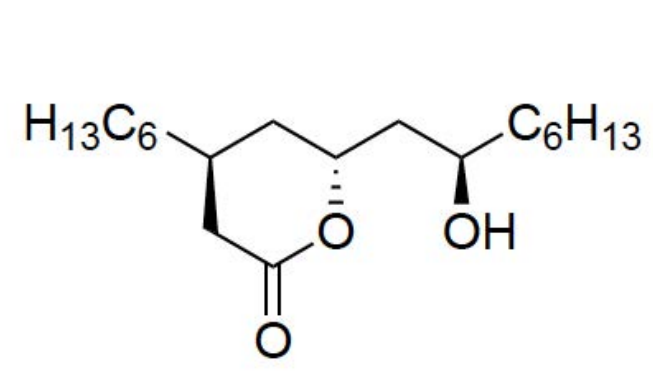

$(R)-9$

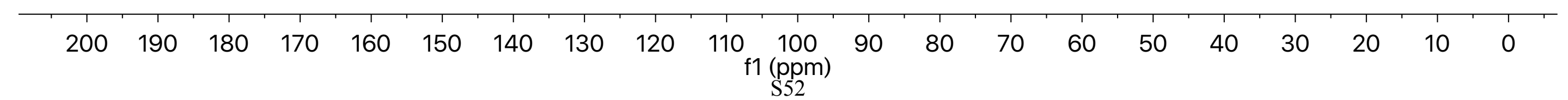


WL-2-073-up-1DNOE

599.926 MHz H1 CSSF_noesy1d in cdc13 (ref. to CDCl3 @ 7.26 ppm), temp 25.8 C -> actual temp =27.0 C, autoxid probe

Processed CSSF experiment.

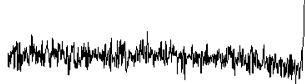

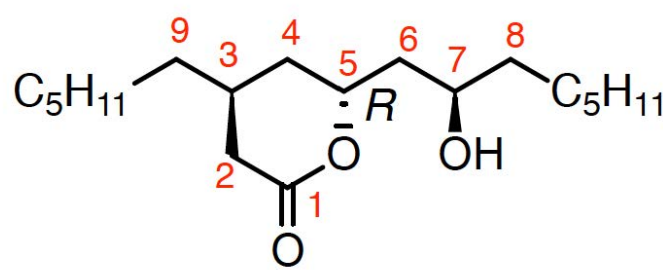

(R)-9

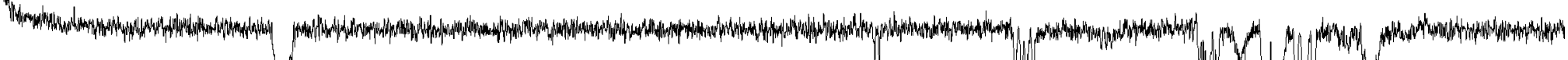

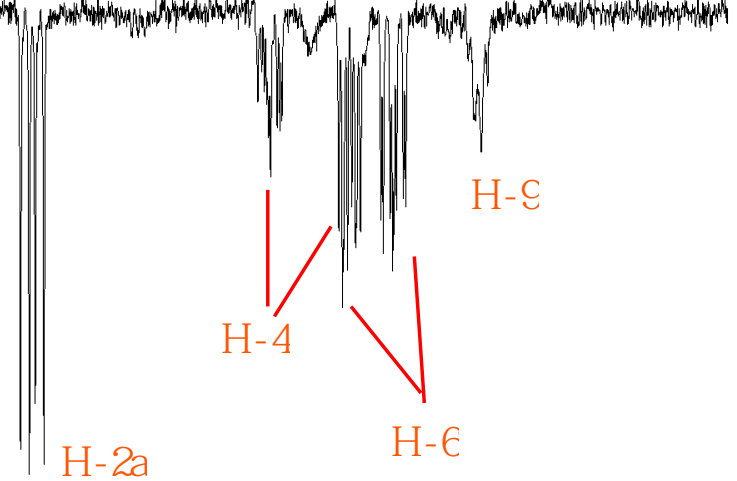

15

5.0

4.5

4.0

3.5

3.0

2.5

2.0

1.5

ppm 
(S)-9-H - WL-2-073-prod-down - 599.926 MHz H1 1D in cdcl3 (ref. to CDCl3 @ 7.26 ppm), temp 25.8 C -> actual temp = 27.0 C, autoxid probe

<smiles>CCCCCCCC(O)CC1CC(C)CC(=O)O1</smiles>

(S)-9

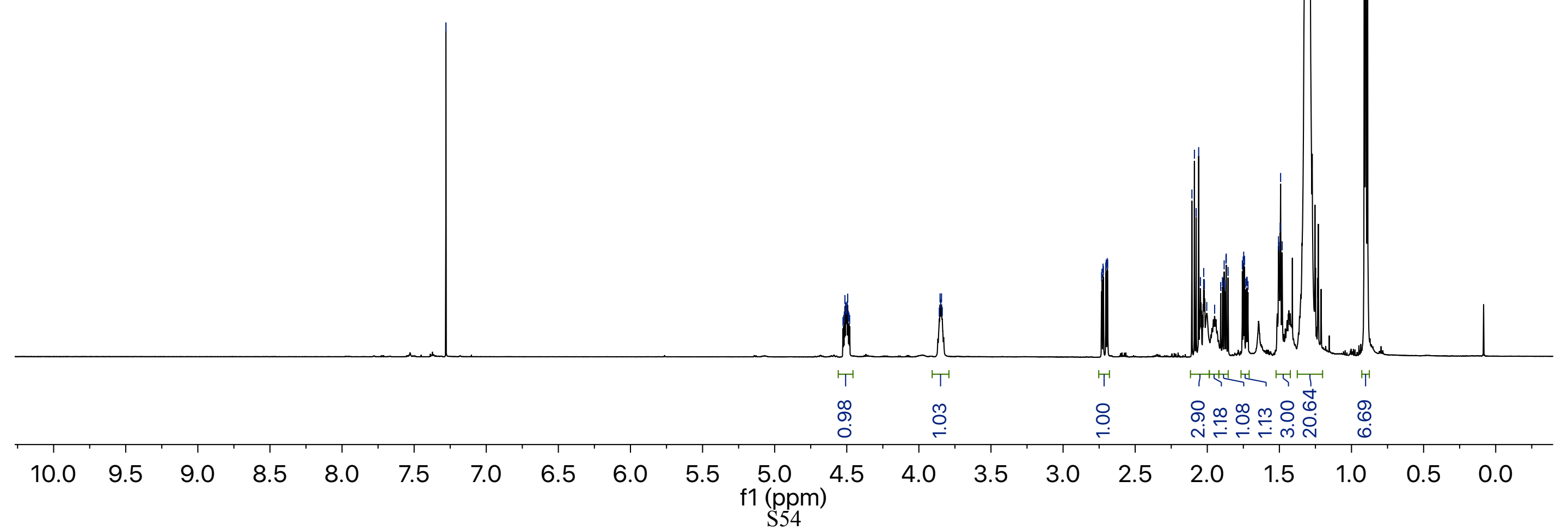


(S)-9-C - WL-2-073-down-CNMR - 150.868 MHz C13[H1] 1D in cdcl3 (ref. to CDCl3 @ 77.06 ppm), temp 25.8 C -> actual temp = 27.0 C, autox (1)

(S)-9

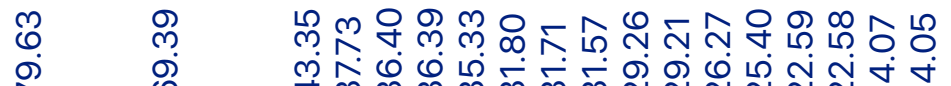

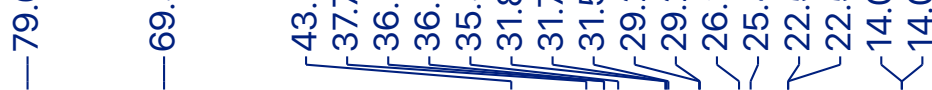

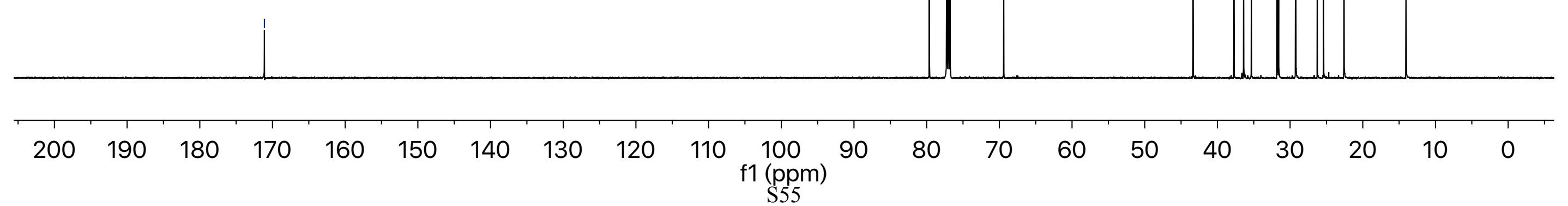


WL-2-073-down-1D-NOE

599.926 MHz H1 CSSF_noesy1d in $\mathrm{cdc} 13$ (ref. to $\operatorname{CDC} 13$ @ $7.26 \mathrm{ppm}$ ), temp $25.8 \mathrm{C} \rightarrow$ actual temp $=27.0 \mathrm{C}$, autoxid probe

date: Feb 132013 sweep width F2/F1: $6010 / 50 \mathrm{~Hz}$

spectrometer: $i 600$

acq.time: $3.0 \mathrm{~s}$ relax.time: $2.0 \mathrm{~s}$ dig.res.F2/F1: $0.1 / 0.1 \mathrm{~Hz} / \mathrm{pt}$

Pulse Sequence:
$\mathrm{hz} / \mathrm{mm} F 2 / \mathrm{FS} 1: 9.9 / 0.3$

File: CSSF_noesy1d

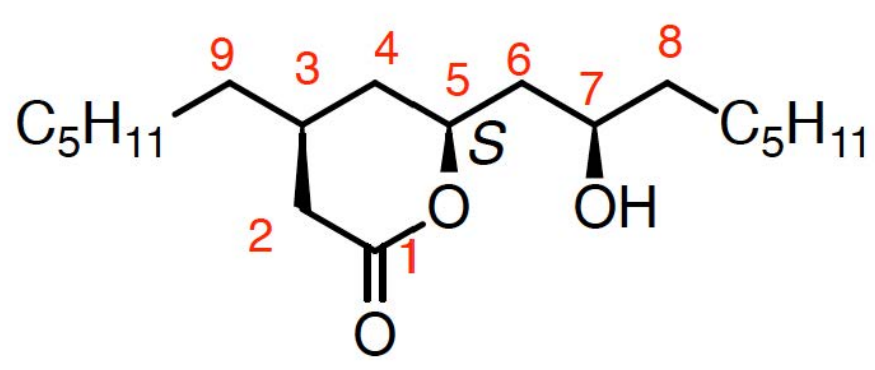

active. 406

A ctivation of $\mathrm{H}-5$

(S)-9



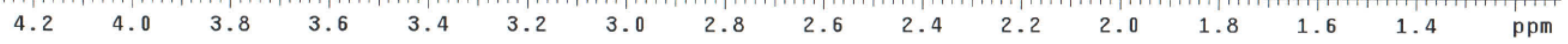




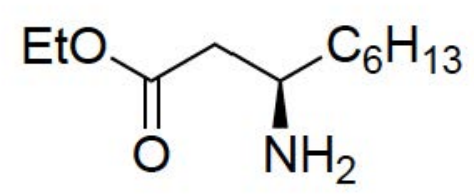

10




10-C - Wl-1-012-pro-CNMR - 125.266 MHz C13[H1] 1D in cdcl3 (ref. to CDCl3 @ 77.06 ppm), temp $26.4 \mathrm{C}->$ actual temp = $27.0 \mathrm{C}$, autoxdb pro<smiles>CCOC(=O)C[C@H](N)c1ccccc1</smiles>

10

$\begin{array}{lllllllllllllllllllll}200 & 190 & 180 & 170 & 160 & 150 & 140 & 130 & 120 & 110 & \begin{array}{c}100 \\ \mathrm{f} 1 \text { (ppm) }\end{array} & 80 & 70 & 60 & 50 & 40 & 30 & 20 & 10 & 0 & -10\end{array}$


11-H - WL-1-030-product - 399.794 MHz H1 1D in cdcl3 (ref. to CDCl3 @ 7.26 ppm), temp 27.0 C -> actual temp = 27.0 C, autoxdb probe -

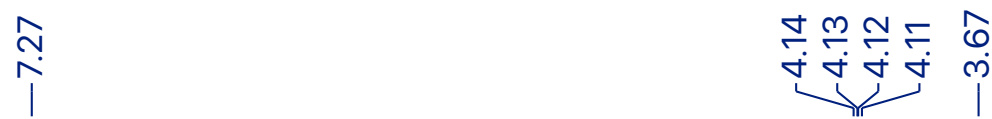

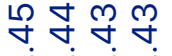

isin

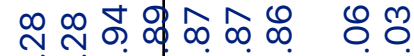
نंioi io<smiles>COC(=O)CC([18OH])[18OH]</smiles>

11

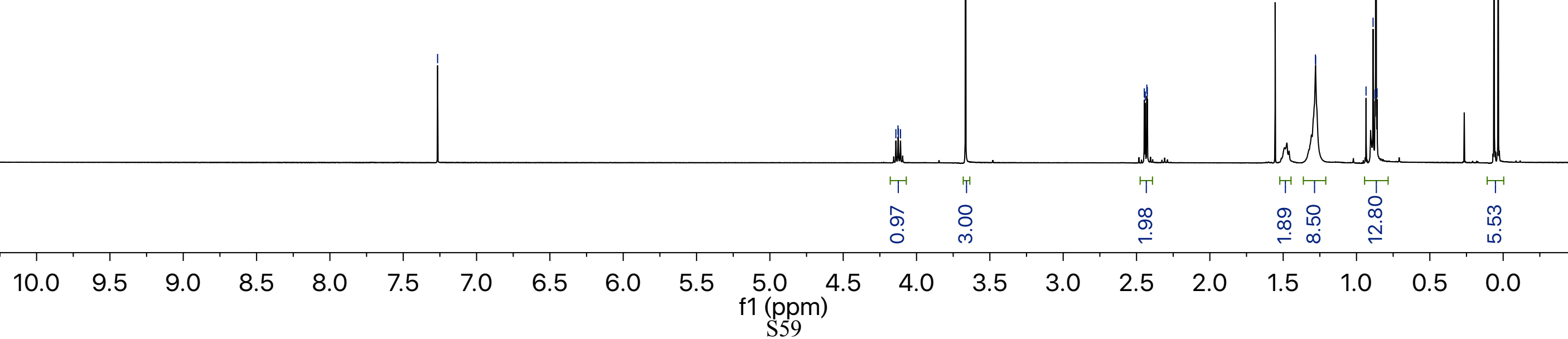


11-C - WL-1-030-CNMR - 100.537 MHz C13[H1] 1D in cdcl3 (ref. to CDCl3 @ 77.06 ppm), temp 27.0 C -> actual temp = 27.0 C, autoxdb probe -
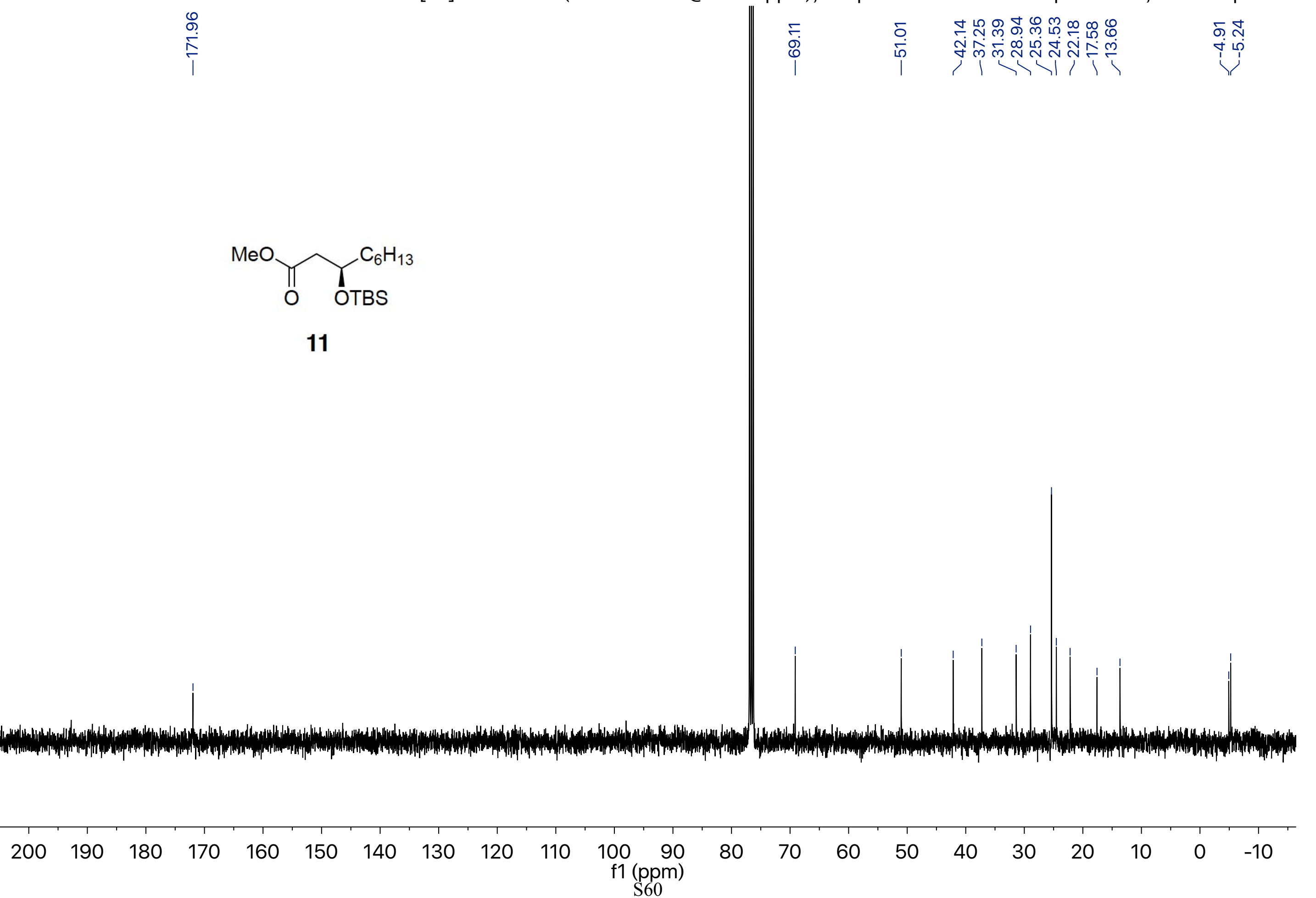
12-H - WL-1-052-product - 399.794 MHz H1 1D in cdcl3 (ref. to CDCl3 @ 7.26 ppm), temp 27.0 C -> actual temp=27.0 C, autoxdb probe — กิ

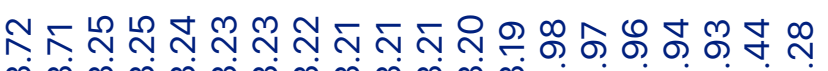

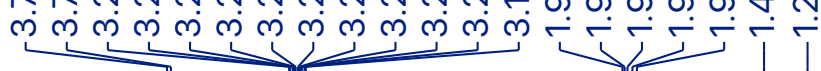

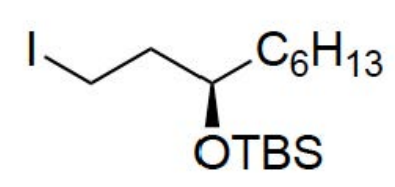

12

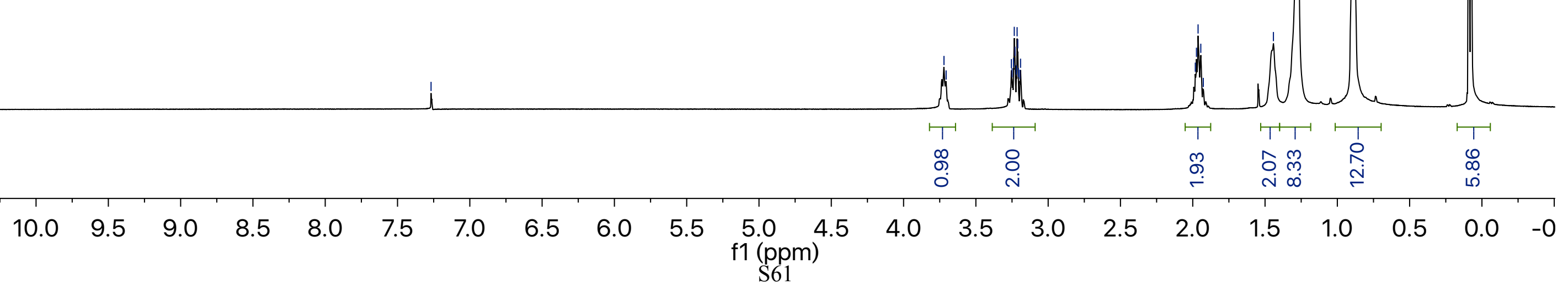


12-C - WL-1-052-CNMR - 100.537 MHz C13[H1] 1D in cdcl3 (ref. to CDCl3 @ 77.06 ppm), temp 27.0 C -> actual temp = 27.0 C, autoxdb probe -

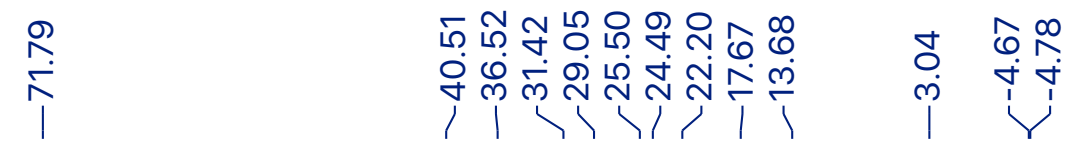

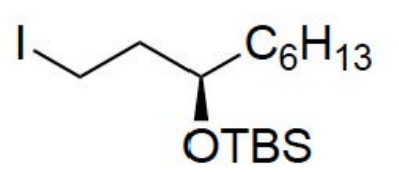

12

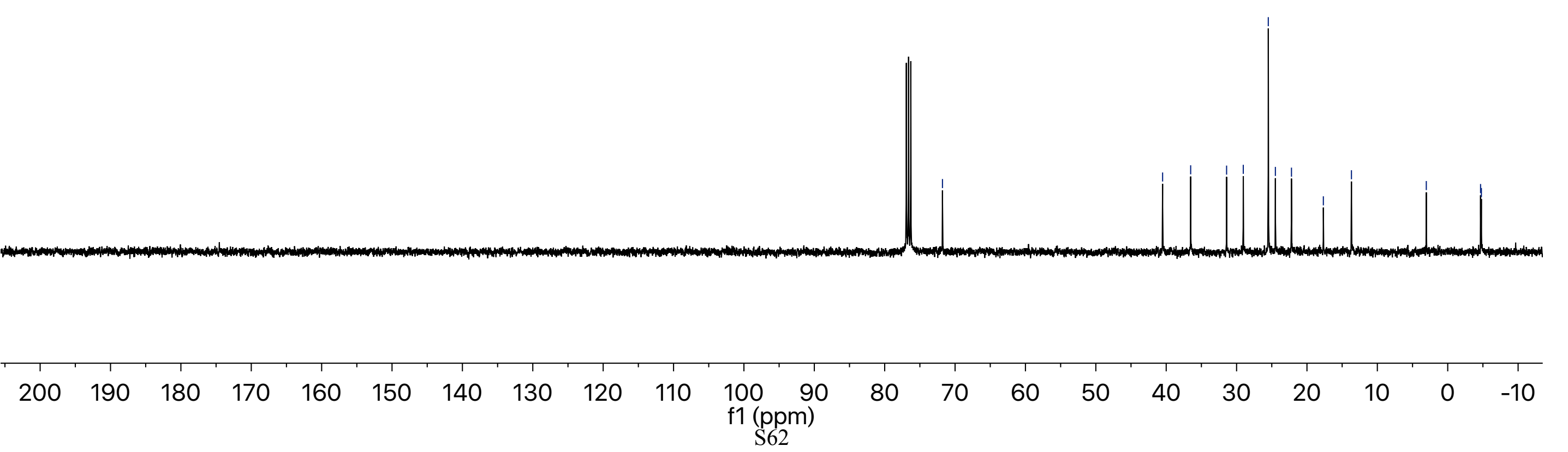


13-H - WL-2-044-product — 599.926 MHz H1 1D in cdcl3 (ref. to CDCl3 @ 7.26 ppm), temp 25.8 C -> actual temp = $27.0 \mathrm{C}$, autoxid probe —

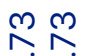

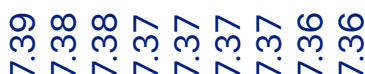

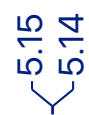

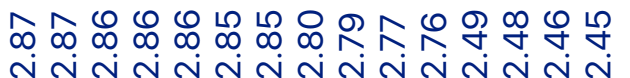
감 守
ij

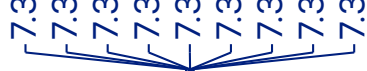

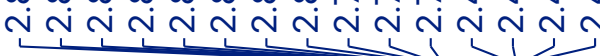

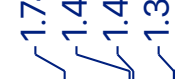

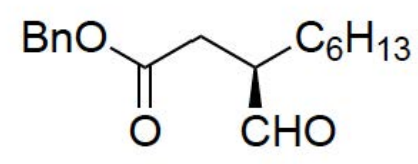

13

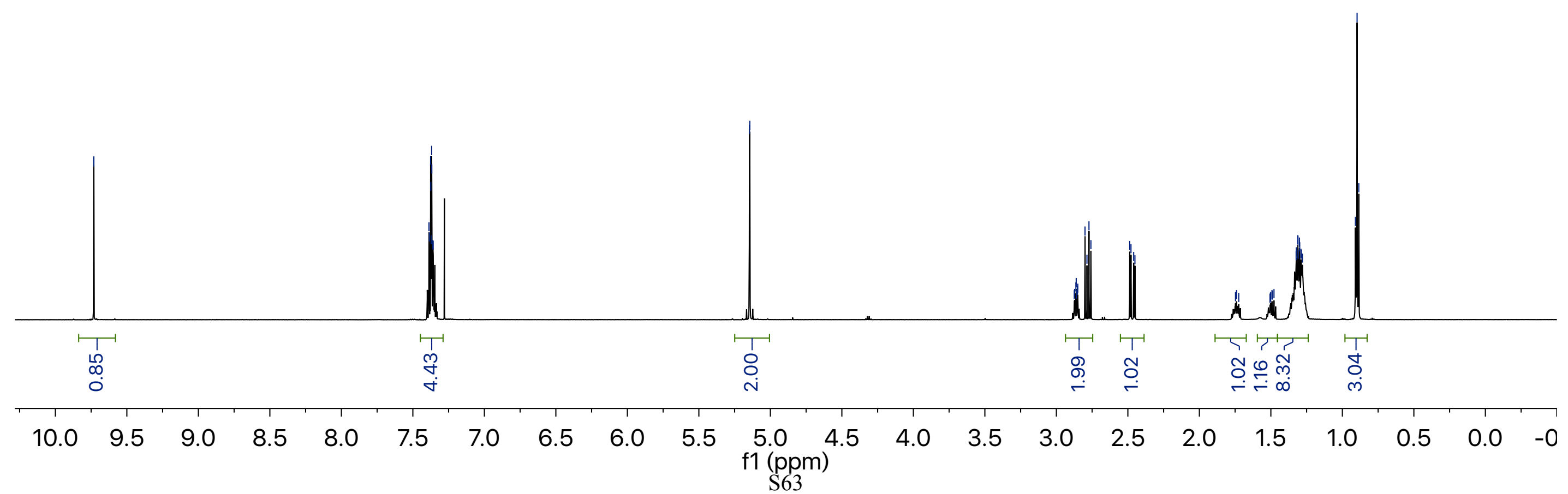


13-C - WL-1-090-CNMR - 100.539 MHz C13[H1] 1D in cdcl3 (ref. to CDCl3 @ 77.06 ppm), temp 27.0 C -> actual temp = 27.0 C, autoxdb probe -
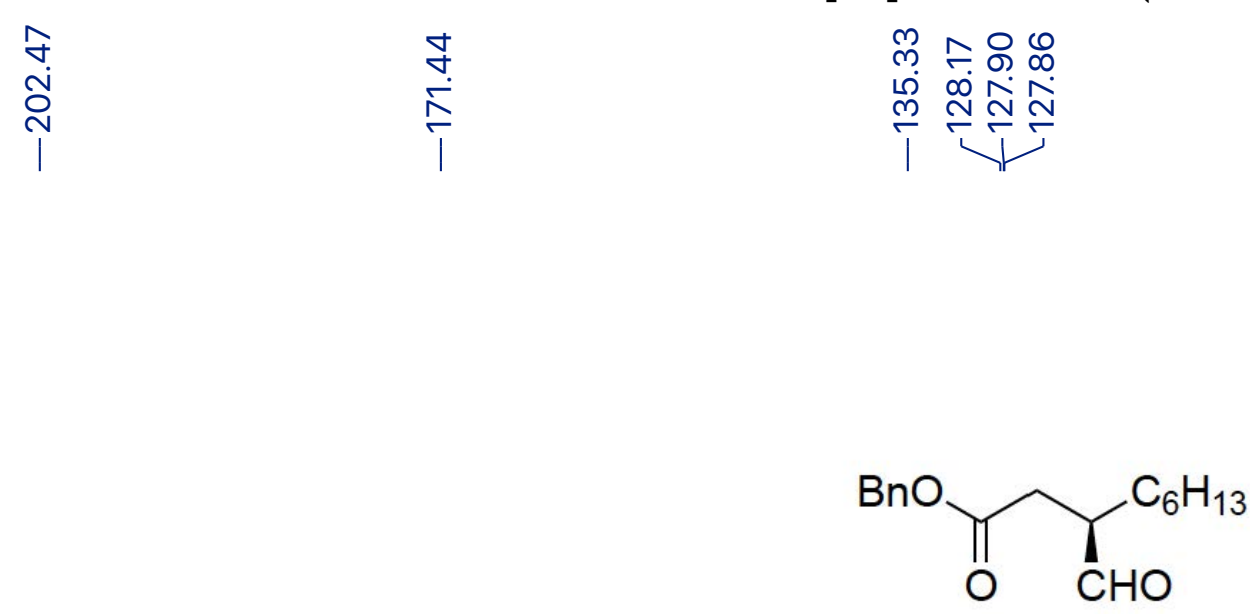

13

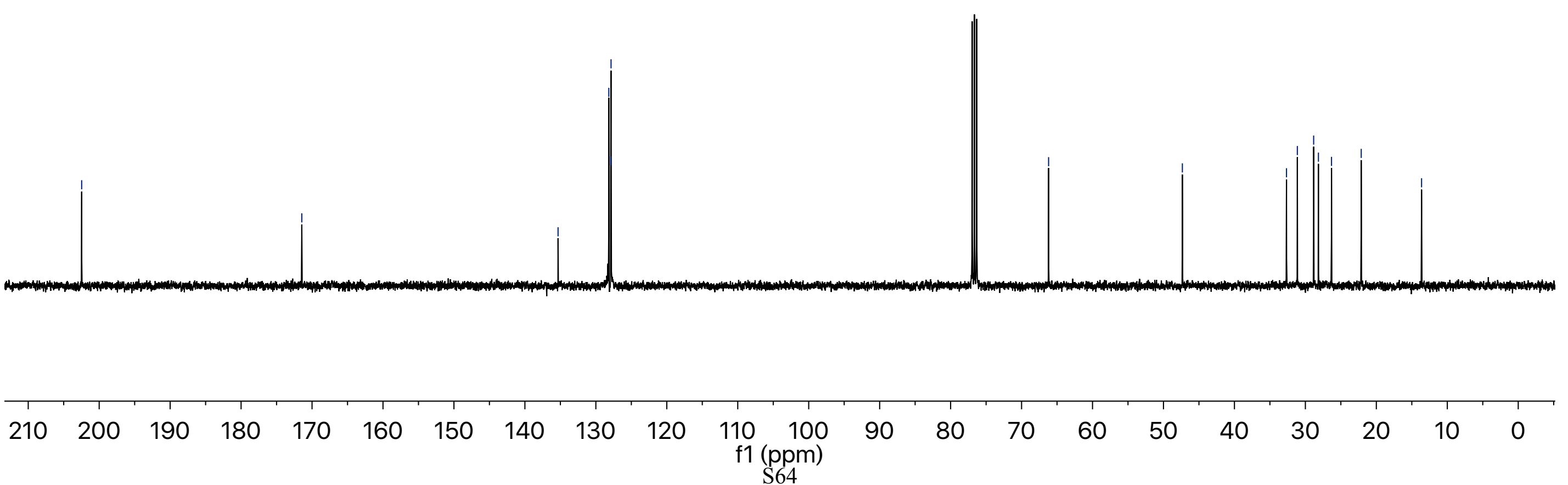




15-H - WL-1-019-product-1HNMR - 399.794 MHz H1 1D in cdcl3 (ref. to CDCl3 @ 7.26 ppm), temp 27.0 C -> actual temp = 27.0 C, autoxdb prok $\stackrel{\substack{i \\ i}}{i}$<smiles>COC(=O)C[C@@H](O)c1ccccc1</smiles>

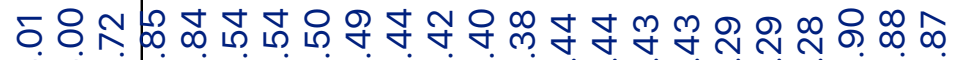

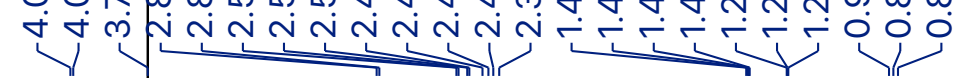

15

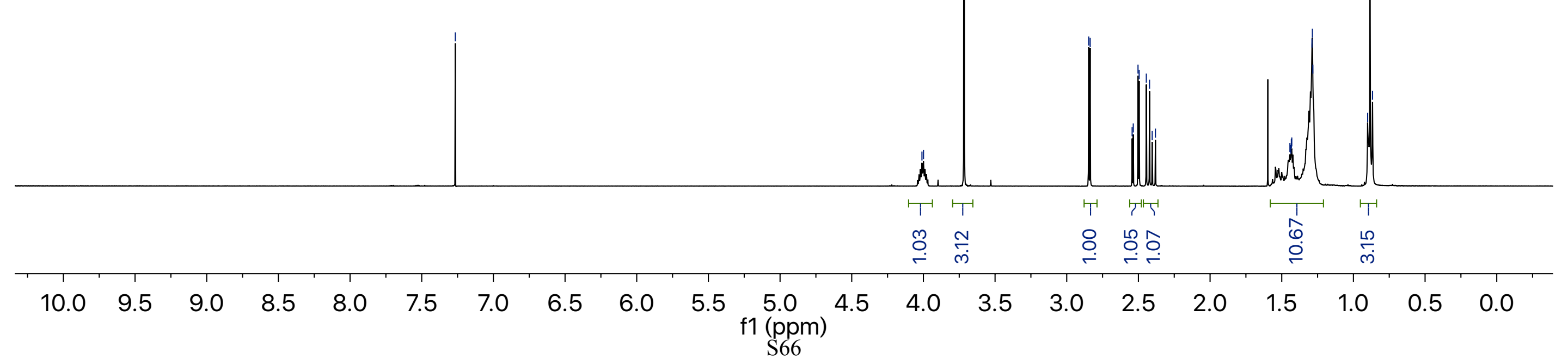


<smiles>COC(=O)C[C@H](O)c1ccccc1</smiles>

15

$\begin{array}{llllllllll}200 & 190 & 180 & 170 & 160 & 150 & 140 & 130 & 120 & 110 \begin{array}{l}100 \\ \mathrm{f} 1(\mathrm{ppm})\end{array}\end{array}$




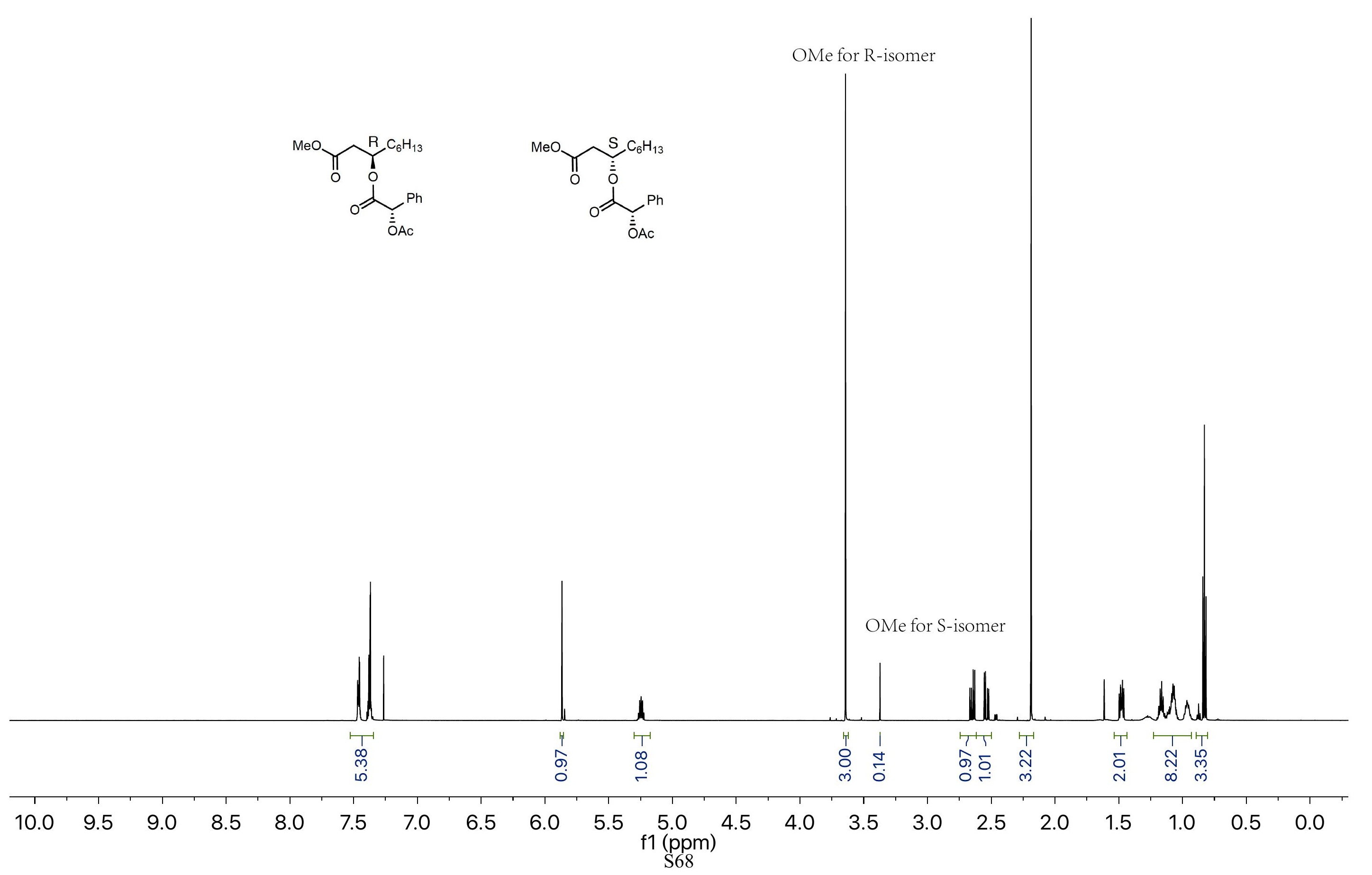


16-H - WL-1-032-product — 399.794 MHz H1 1D in cdcl3 (ref. to CDCl3 @ 7.26 ppm), temp 27.0 C -> actual temp = 27.0 C, autoxdb probe —

ì

舟

ง ำง ง ง

16

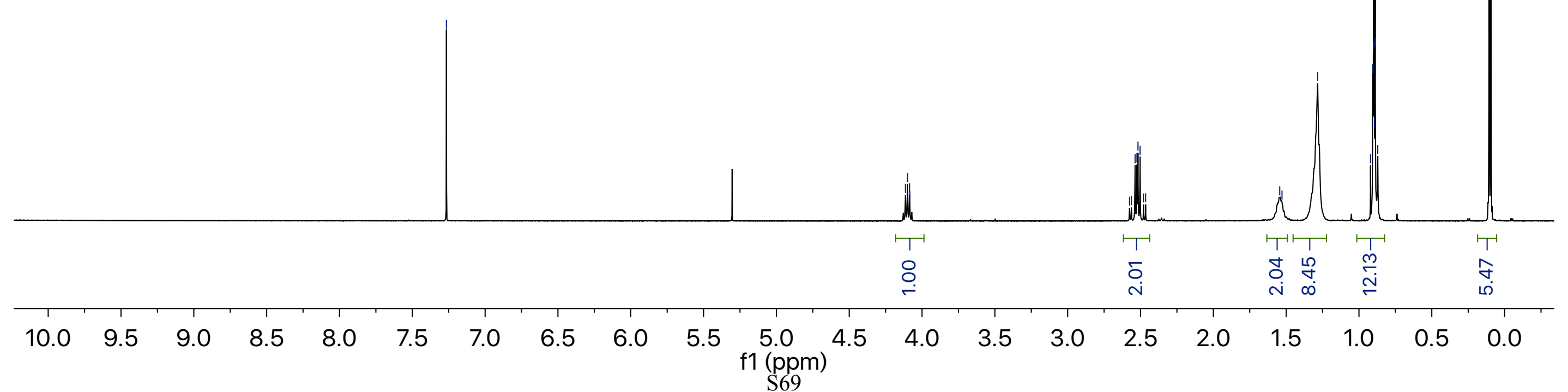


16-C - WL-1-032-CNMR - 100.537 MHz C13[H1] 1D in cdcl3 (ref. to CDCl3 @ 77.06 ppm), temp 27.0 C -> actual temp = 27.0 C, autoxdb probe -






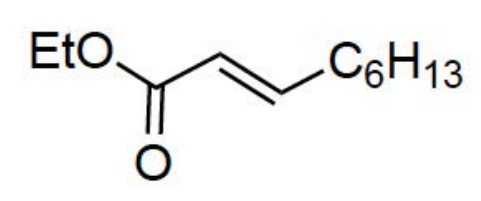

18

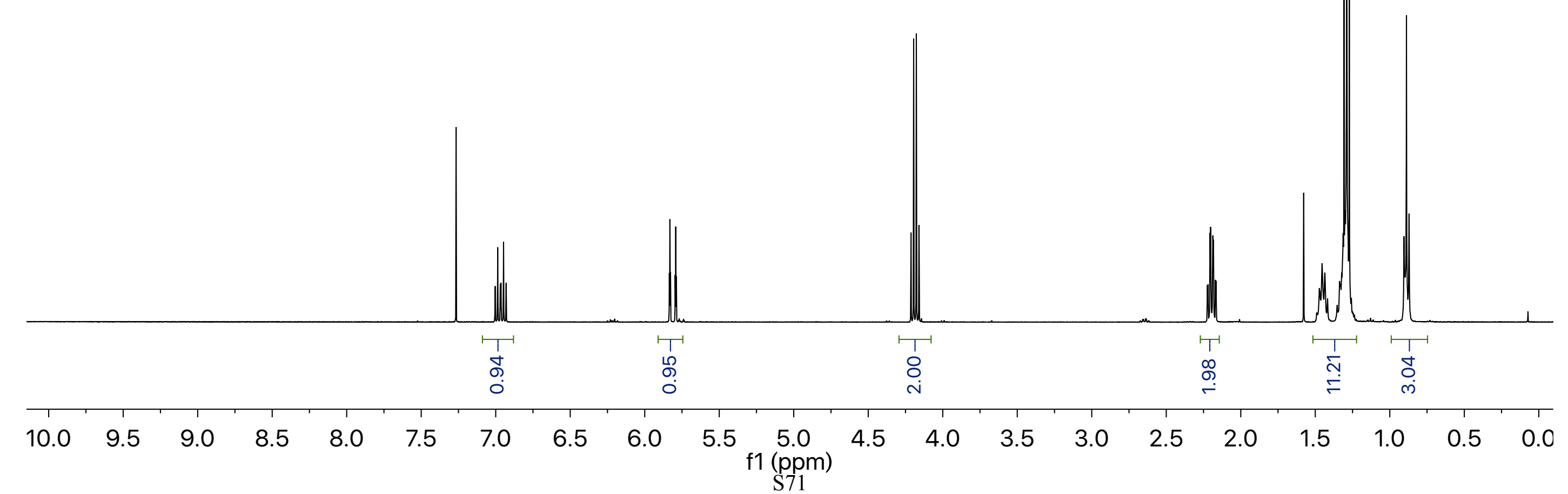


18-C - WL-1-09-E-product-CNMR - 100.537 MHz C13[H1] 1D in cdcl3 (ref. to CDCl3 @ 77.06 ppm), temp $27.0 \mathrm{C}->$ actual temp = 27.0 C, autoxı
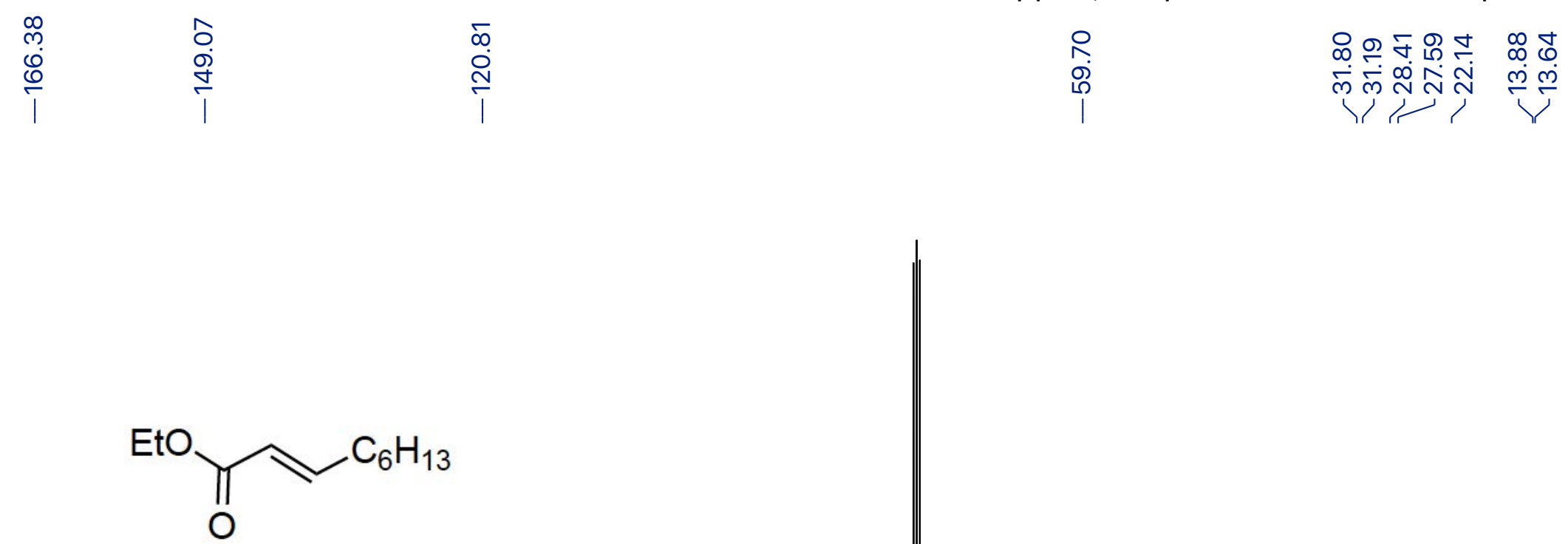

18

\begin{tabular}{|c|c|c|c|c|c|c|c|c|c|c|c|c|c|c|c|c|c|c|c|c|}
\hline 200 & 190 & 180 & 170 & 160 & 150 & 140 & 130 & 120 & 110 & $\begin{array}{c}100 \\
\mathrm{f} 1(\mathrm{ppm}) \\
\mathrm{S} 72\end{array}$ & 90 & 80 & 70 & 60 & 50 & 40 & 30 & 20 & 10 & 0 \\
\hline
\end{tabular}




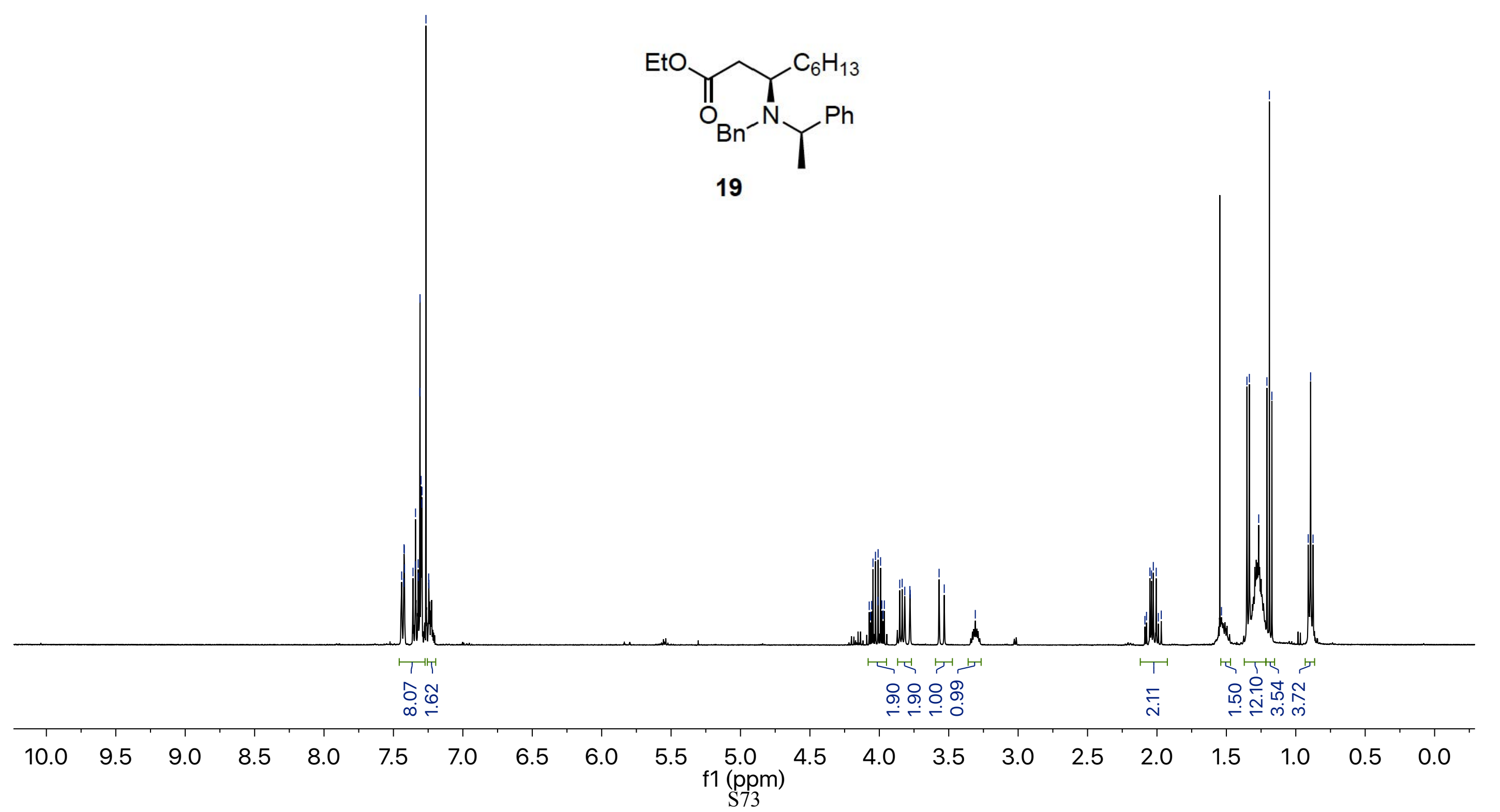


19-C - WL-addition-CNMR - 100.537 MHz C13[H1] 1D in cdcl3 (ref. to CDCl3 @ 77.06 ppm), temp $27.0 \mathrm{C}->$ actual temp = 27.0 C, autoxdb probı
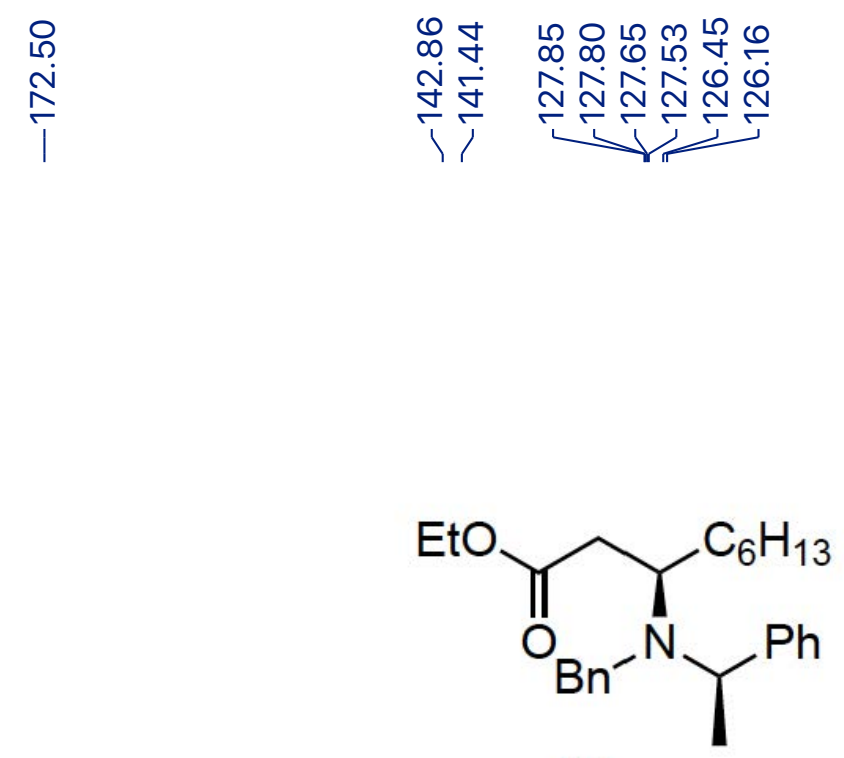

19

0

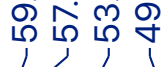

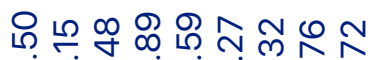

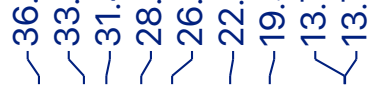

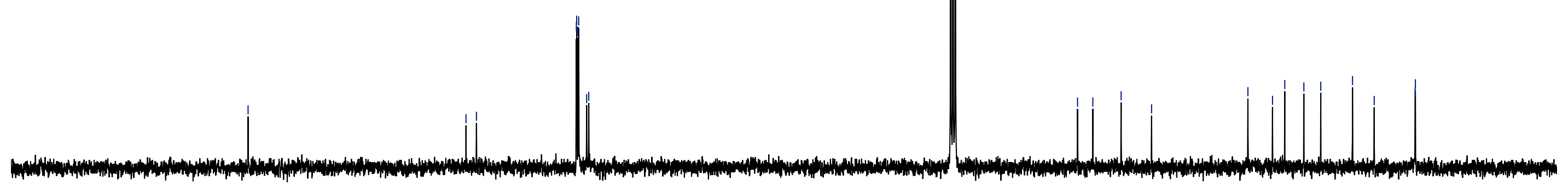

$\begin{array}{llllllllllllllllllllll}200 & 190 & 180 & 170 & 160 & 150 & 140 & 130 & 120 & 110 & \begin{array}{c}100 \\ \mathrm{f} 1(\mathrm{ppm})\end{array} & 90 & 80 & 70 & 60 & 50 & 40 & 30 & 20 & 10 & 0 & \end{array}$




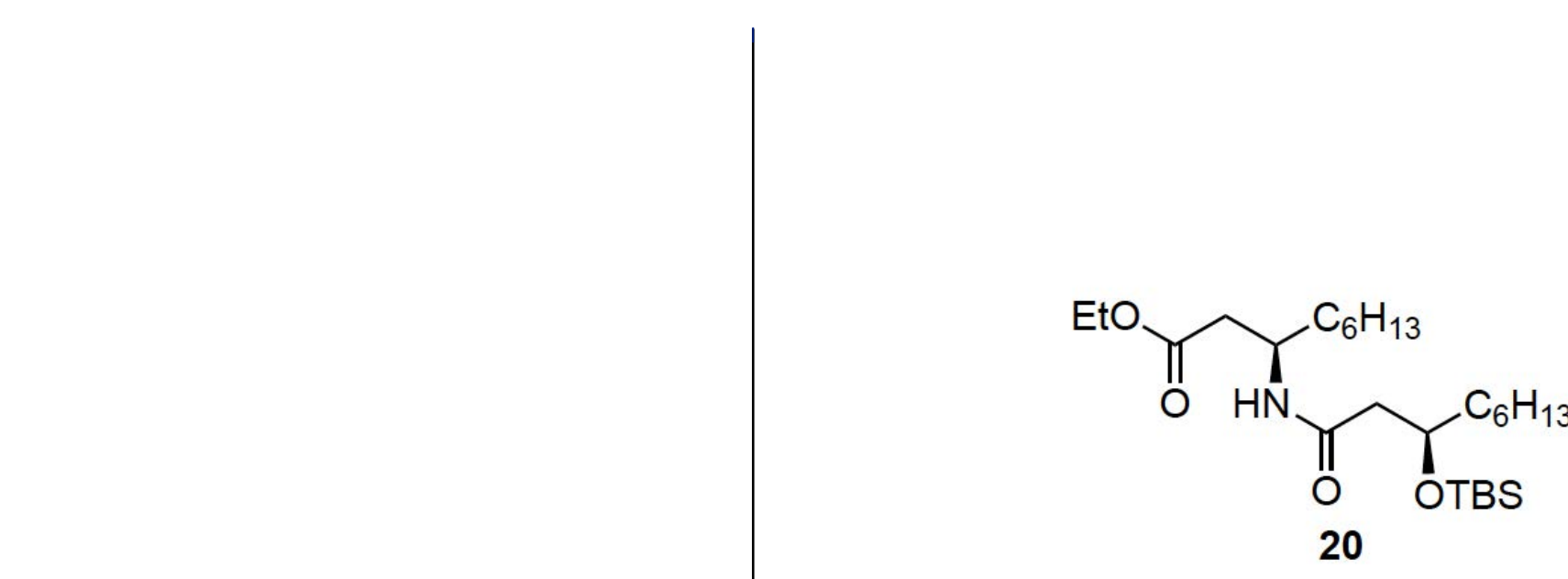

20

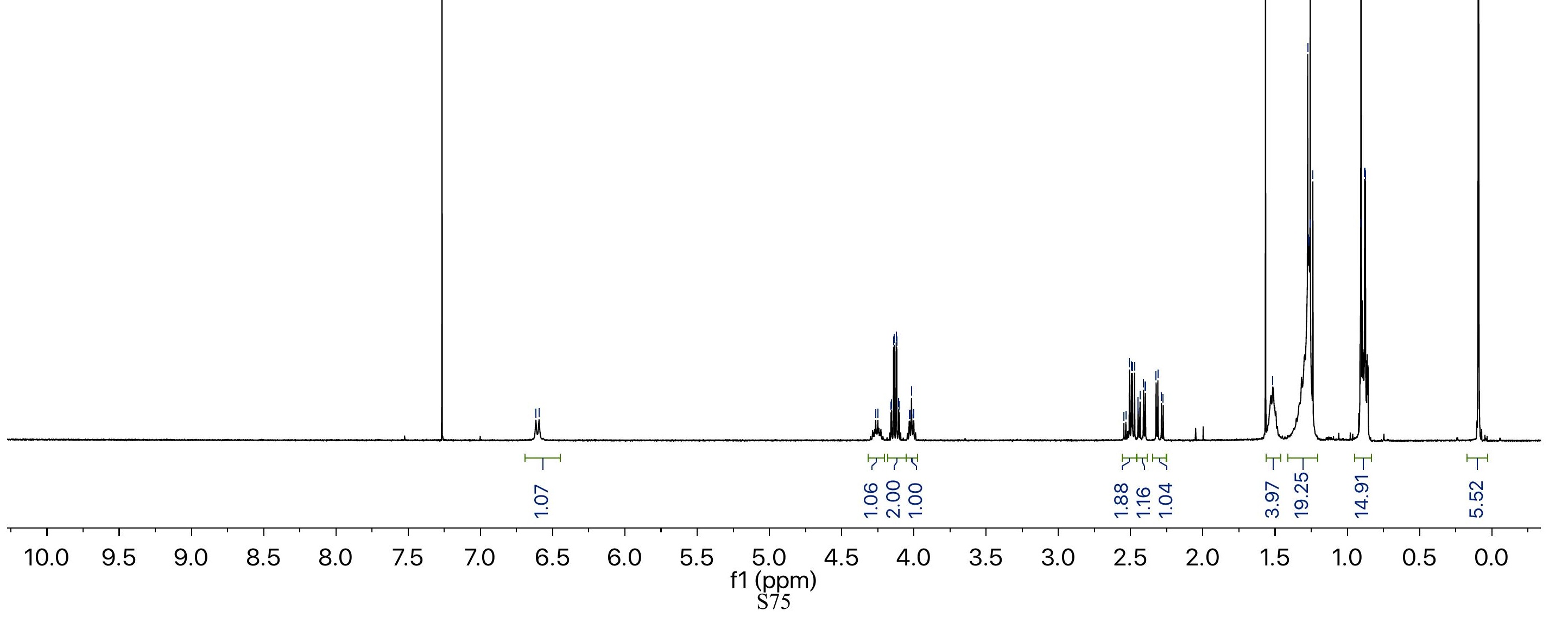


20-C - WL-1-033-CNMR - 100.537 MHz C13[H1] 1D in cdcl3 (ref. to CDCl3 @ 77.06 ppm), temp $27.0 \mathrm{C}->$ actual temp = 27.0 C, autoxdb probe .

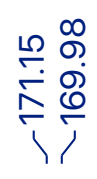

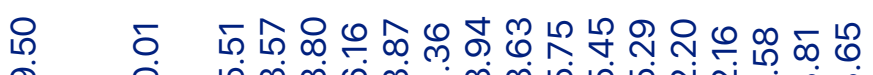

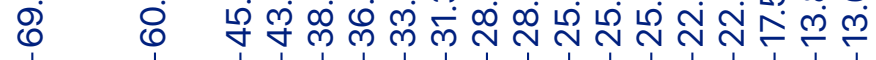<smiles>CCCCCC(CC)CC(=O)NC(CCCCC)CC(=O)OCC</smiles>

20

$\begin{array}{lllllllllll}200 & 190 & 180 & 170 & 160 & 150 & 140 & 130 & 120 & 110 & \begin{array}{l}100 \\ \mathrm{f} 1(\mathrm{ppm})\end{array}\end{array}$


21-H - WL-1-043-product-a - 399.794 MHz H1 1D in cdcl3 (ref. to CDCl3 @ 7.26 ppm), temp 27.0 C -> actual temp = 27.0 C, autoxdb probe -

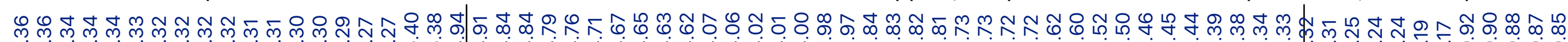

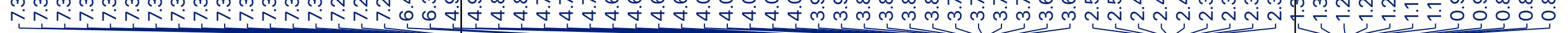

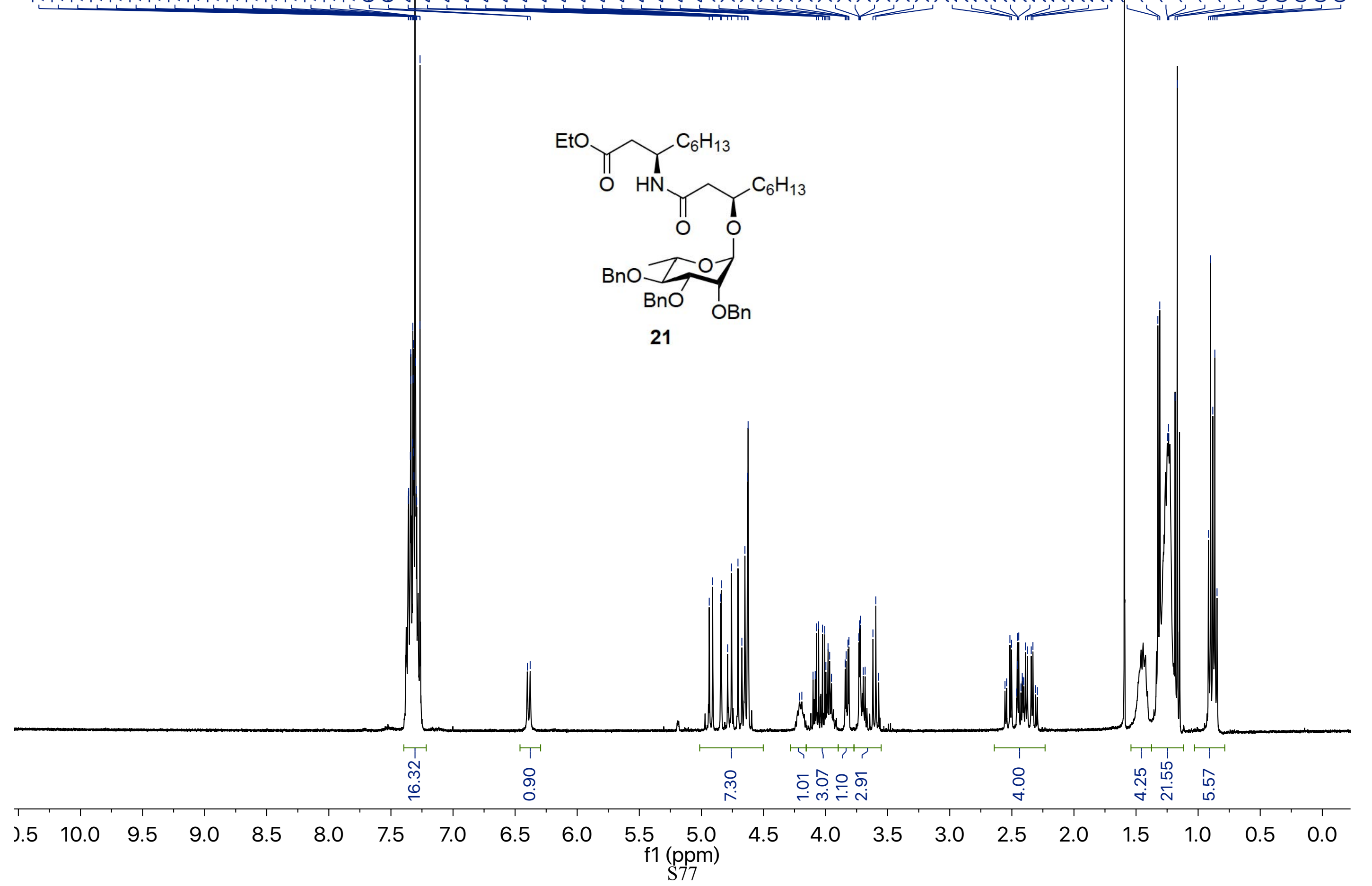


21-C - Lei, WL-1-043-pro - 125.691 MHz C13[H1] 1D in cdcl3 (ref. to CDCl3 @ 77.06 ppm), temp $27.7 \mathrm{C}->$ actual temp = 27.0 C, colddual probe

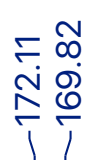
ำ ํํำ



คํํ유.

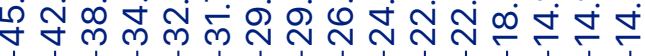

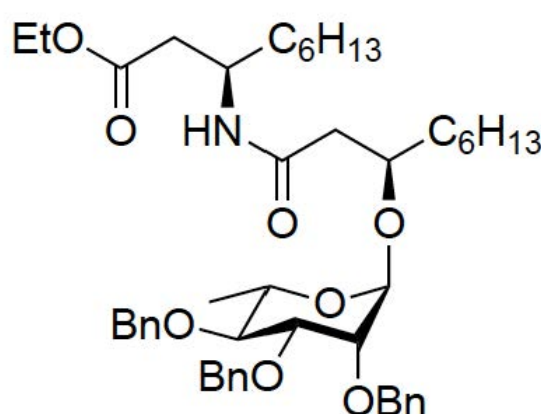

21

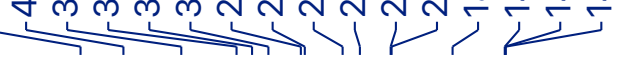






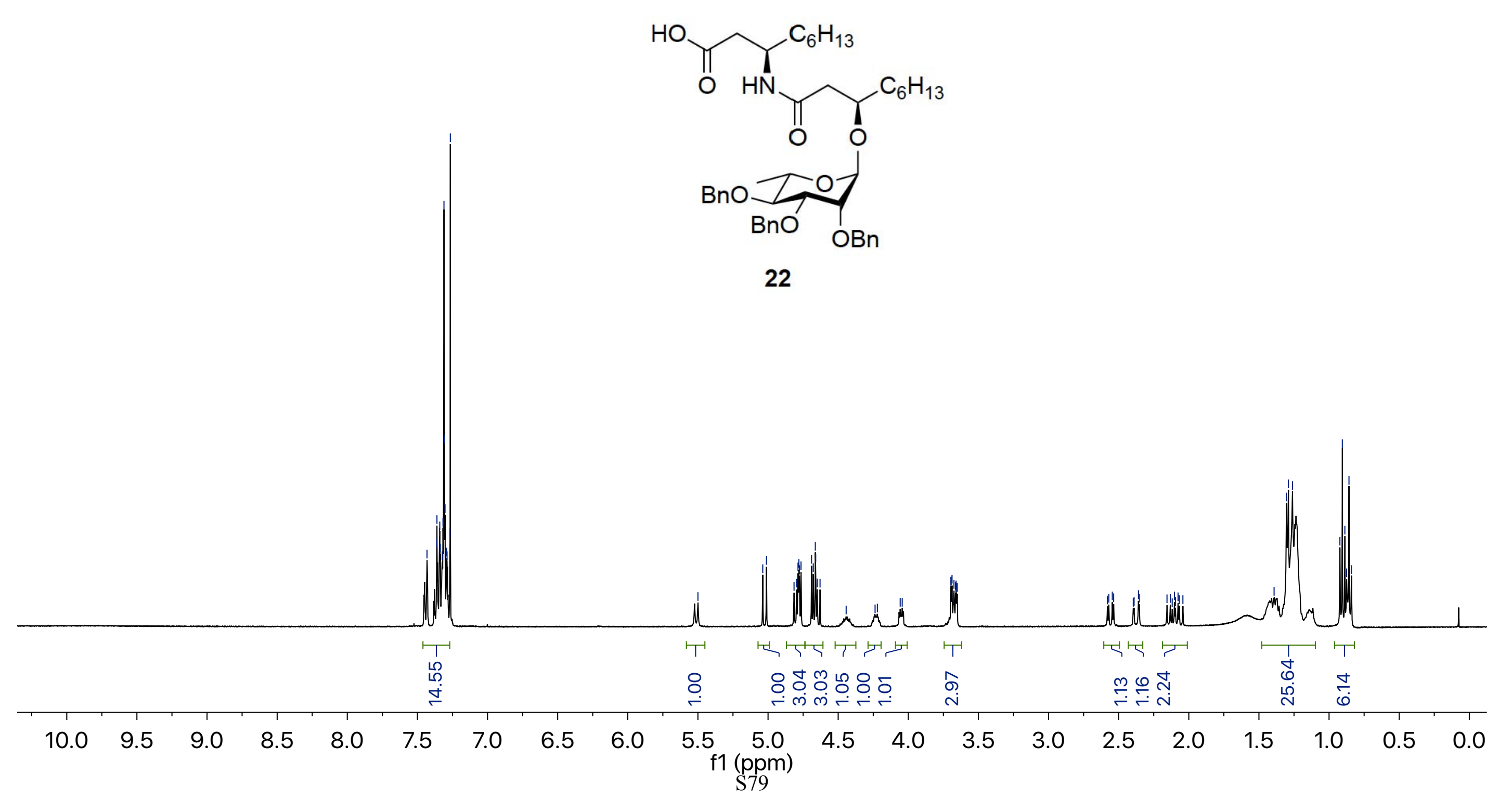


22-C - WL-1-048-CNMR-U500 - 125.690 MHz C13[H1] 1D in cd3od (ref. to CD3OD @ 49.0 ppm), temp 27.7 C -> actual temp = 27.0 C, colddue 峁

$\stackrel{0}{\oplus}$

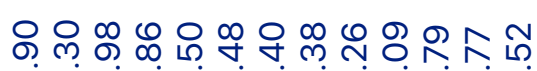

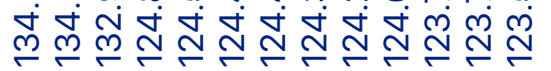

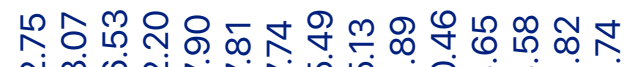

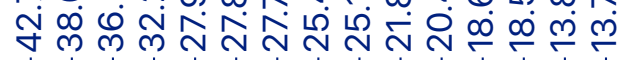

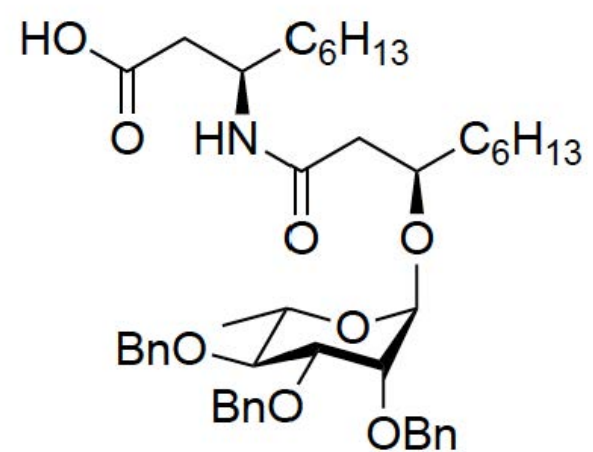

22

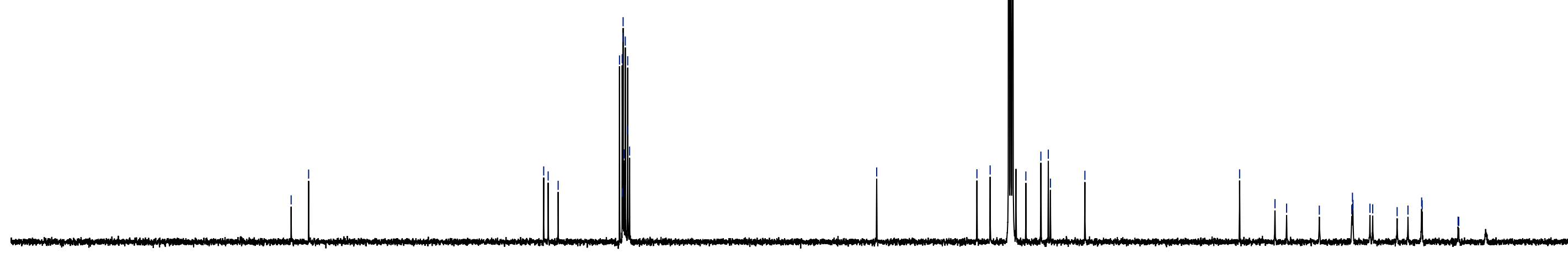


<smiles></smiles>

23

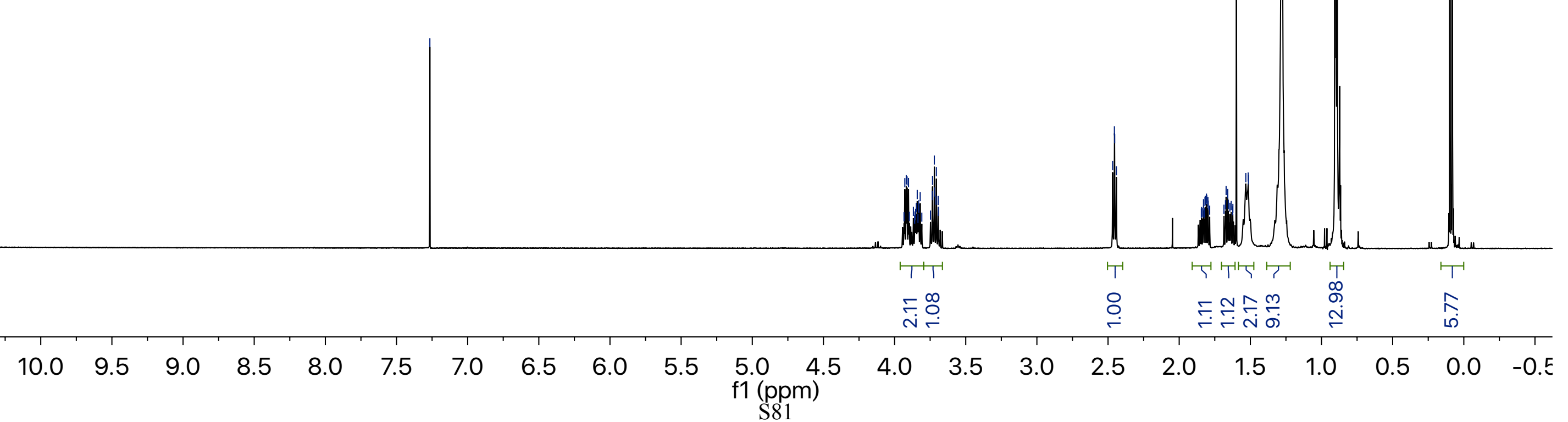


<smiles>OCCC([18OH])[18OH]</smiles> 
24-H - WL-1-064-product — 399.794 MHz H1 1D in cdcl3 (ref. to CDCl3 @ 7.26 ppm), temp 27.0 C -> actual temp = 27.0 C, autoxdb probe -

$\stackrel{\text { }}{i}$

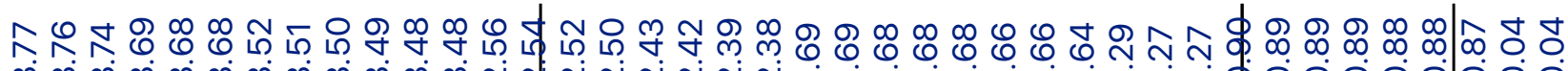

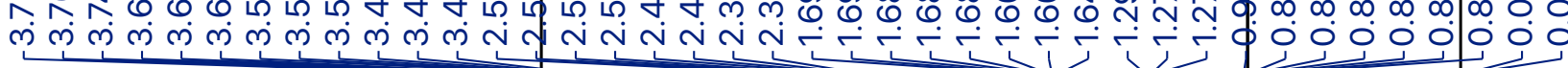

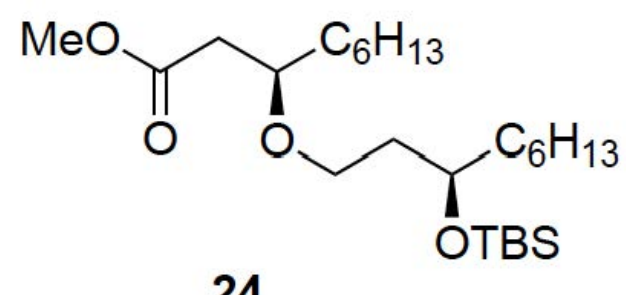

24

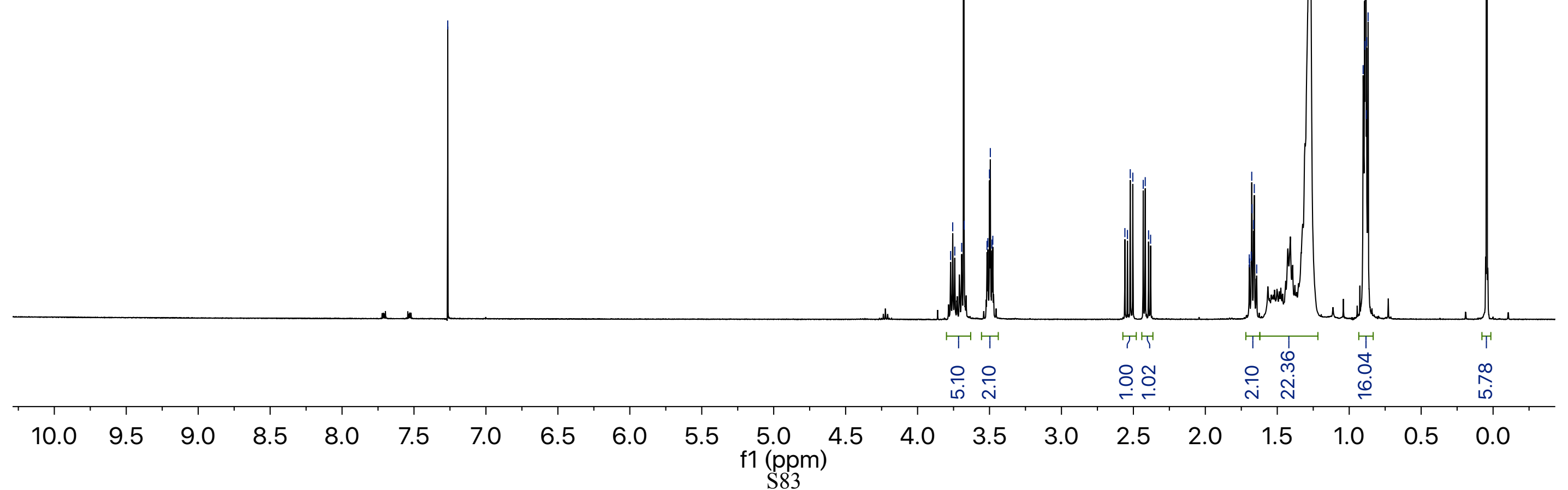



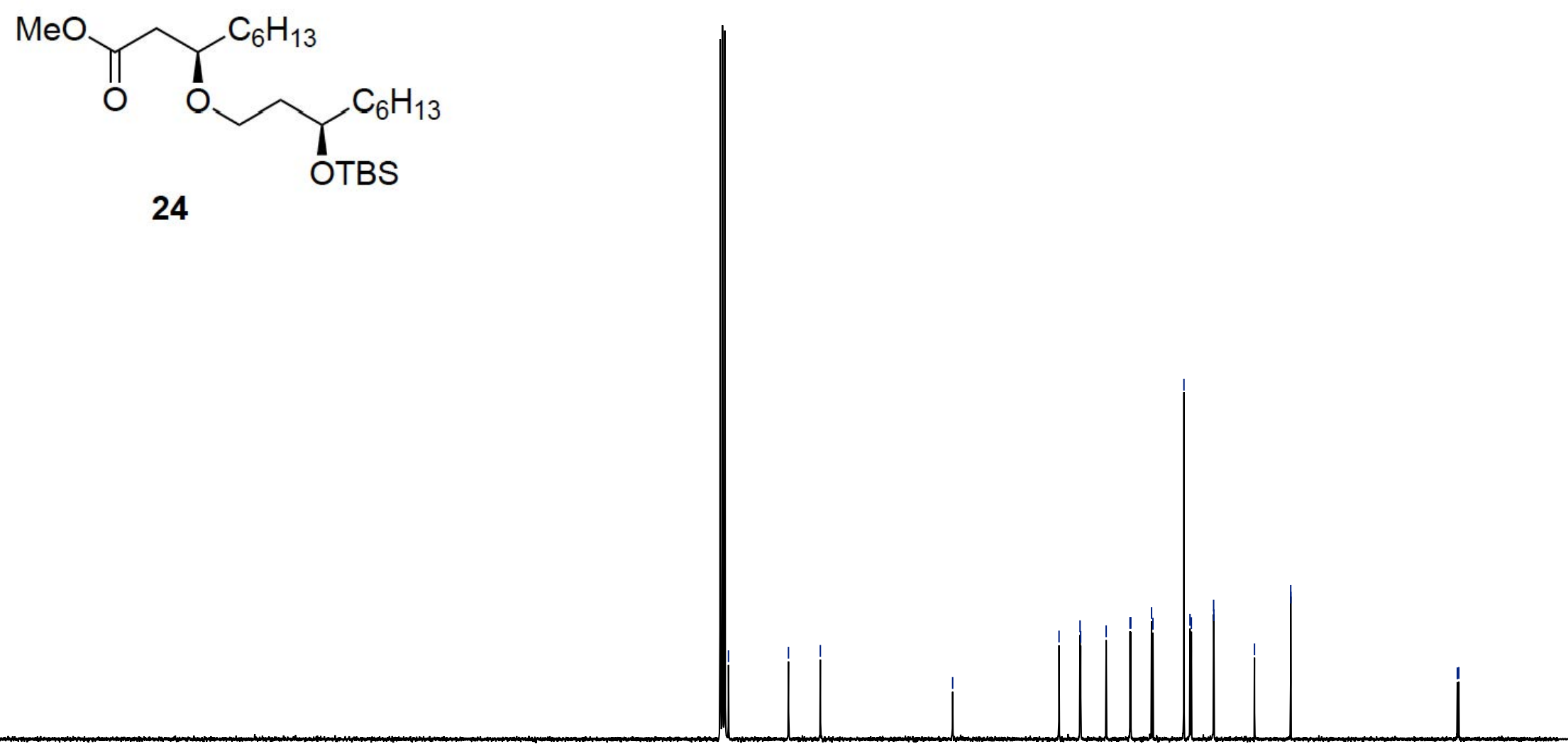

\begin{tabular}{|c|c|c|c|c|c|c|c|c|c|c|c|c|c|c|c|c|c|c|c|c|c|}
\hline 200 & 190 & 180 & 170 & 160 & 150 & 140 & 130 & 120 & 110 & 100 & $\begin{array}{c}90 \\
\text { opm) }\end{array}$ & 80 & 70 & 60 & 50 & 40 & 30 & 20 & 10 & 0 & -10 \\
\hline
\end{tabular}


25-H - WL-1-067-product1 - 599.926 MHz H1 1D in cdcl3 (ref. to CDCl3 @ 7.26 ppm), temp 25.8 C -> actual temp = 27.0 C, autoxid probe -

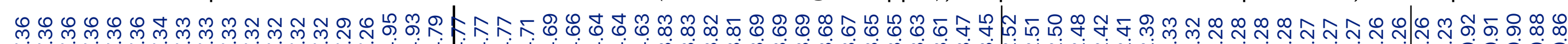

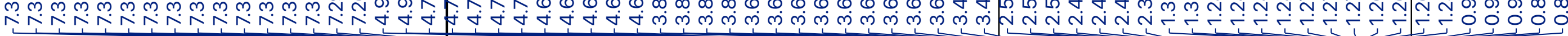

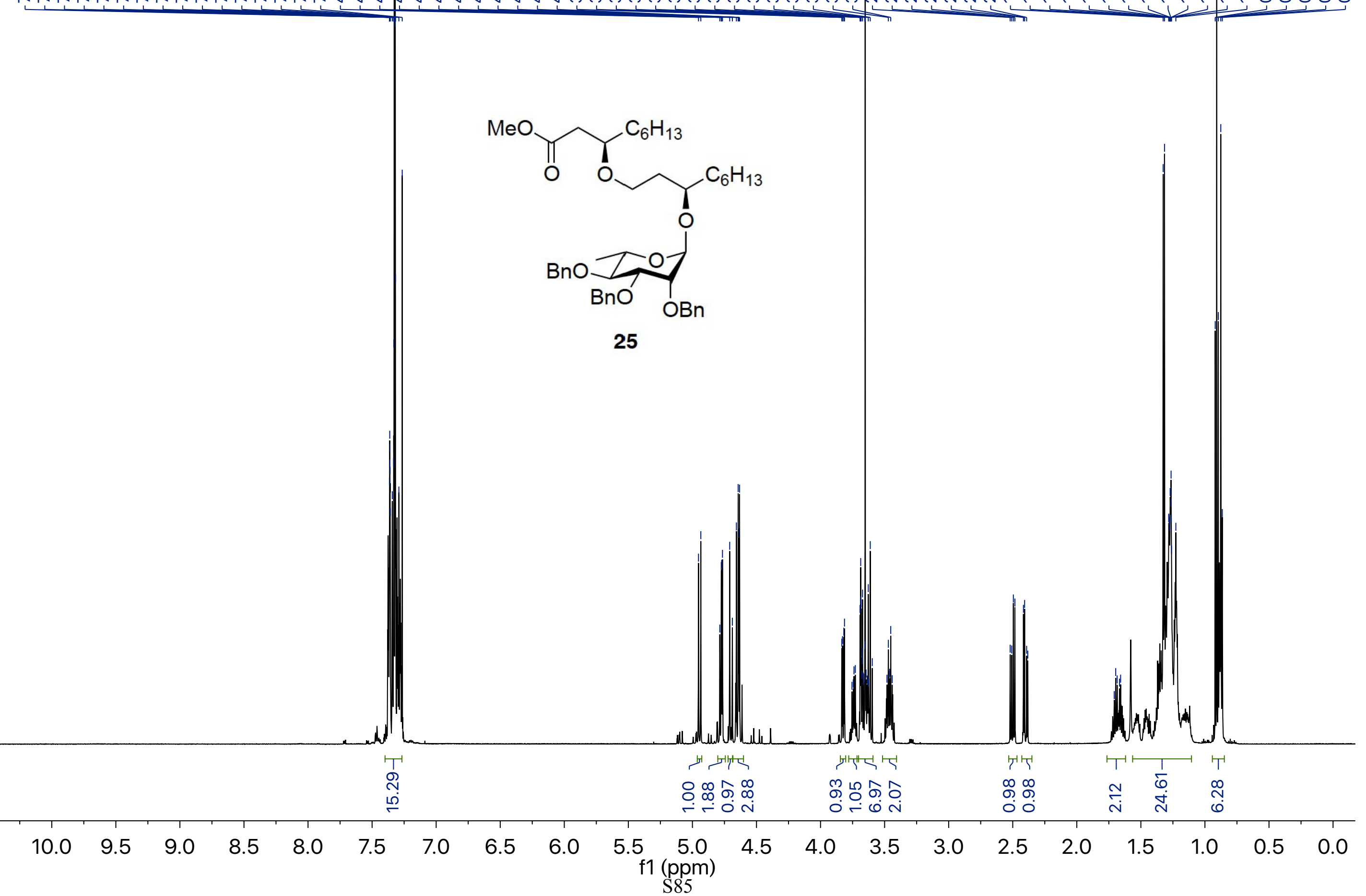


25-C - Lei, WL-1-067 - 125.693 MHz C13[H1] 1D in cdcl3 (ref. to CDCl3 @ 77.06 ppm), temp 27.7 C $->$ actual temp = 27.0 C, colddual probe -

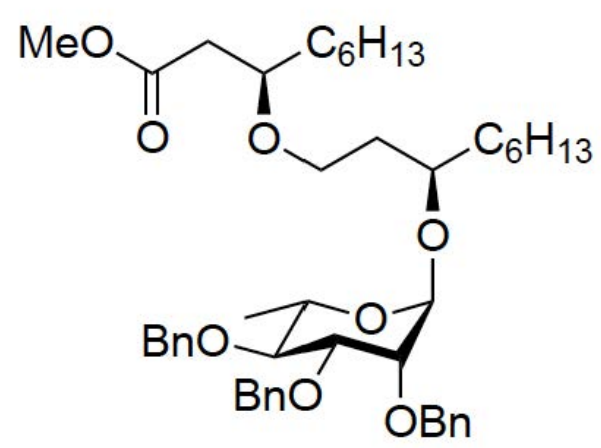

25
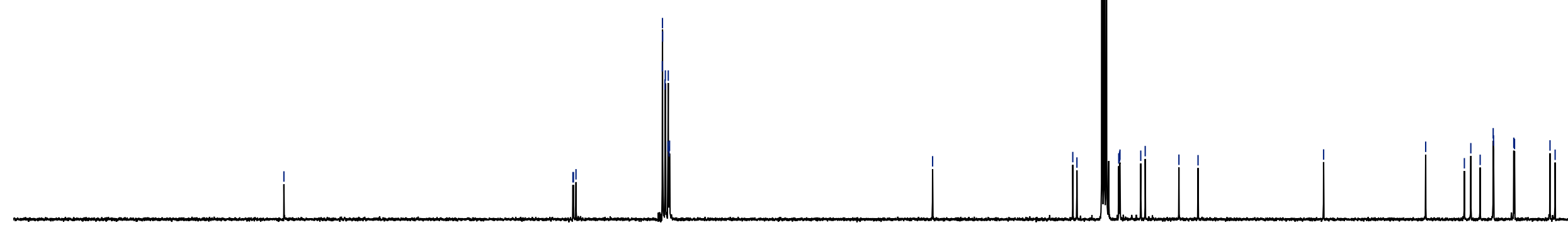


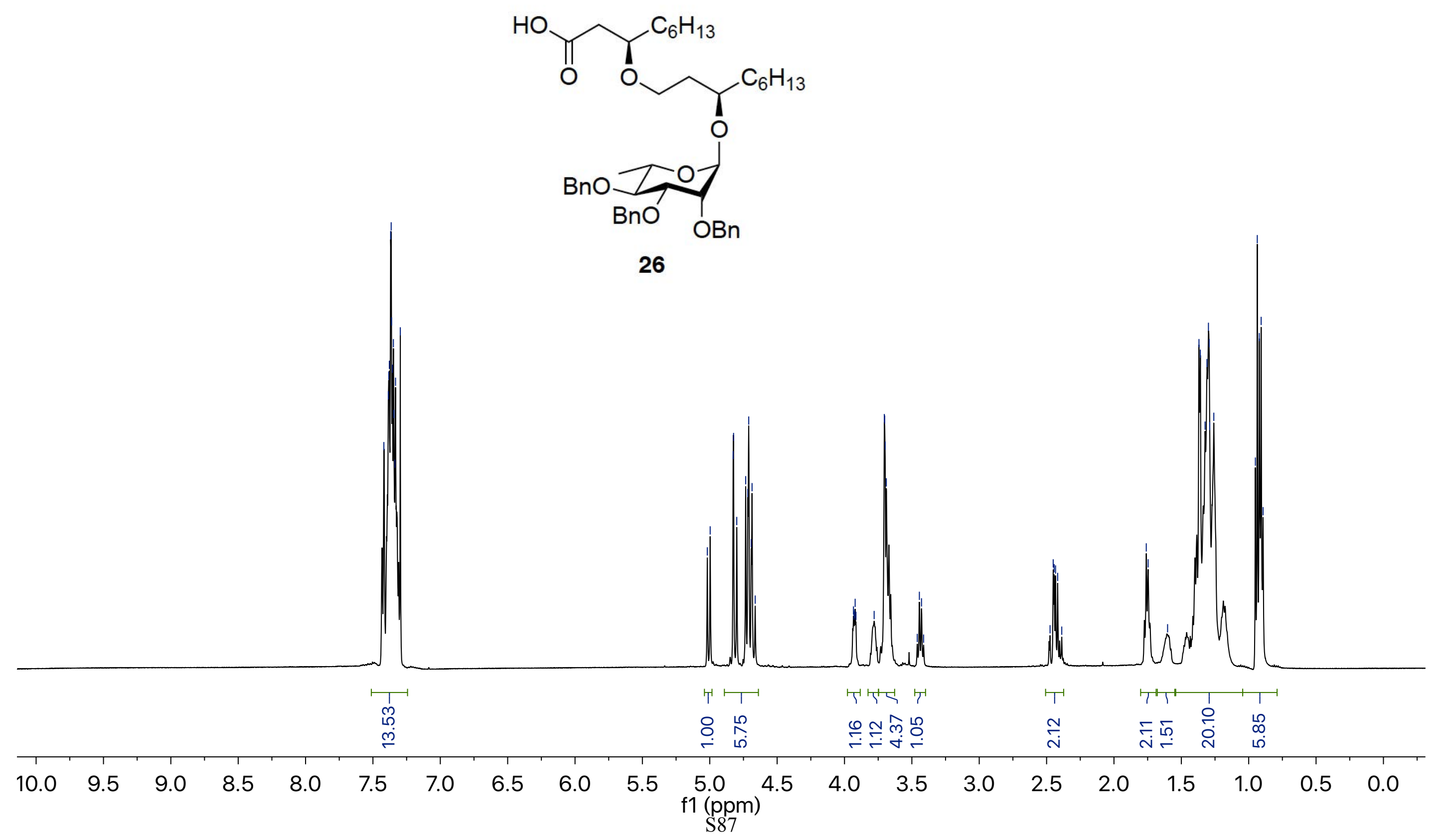


26-C - Lei, WL-1-068-CNMR - 125.693 MHz C13[H1] 1D in cdcl3 (ref. to CDCl3 @ 77.06 ppm), temp $27.7 \mathrm{C}->$ actual temp = 27.0 C, colddual pr

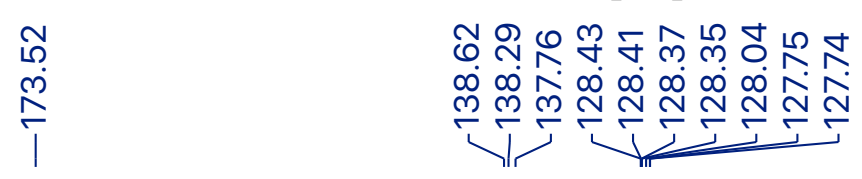

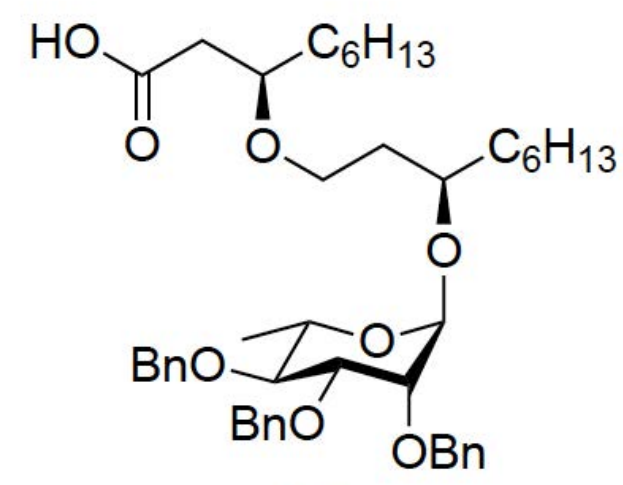

26

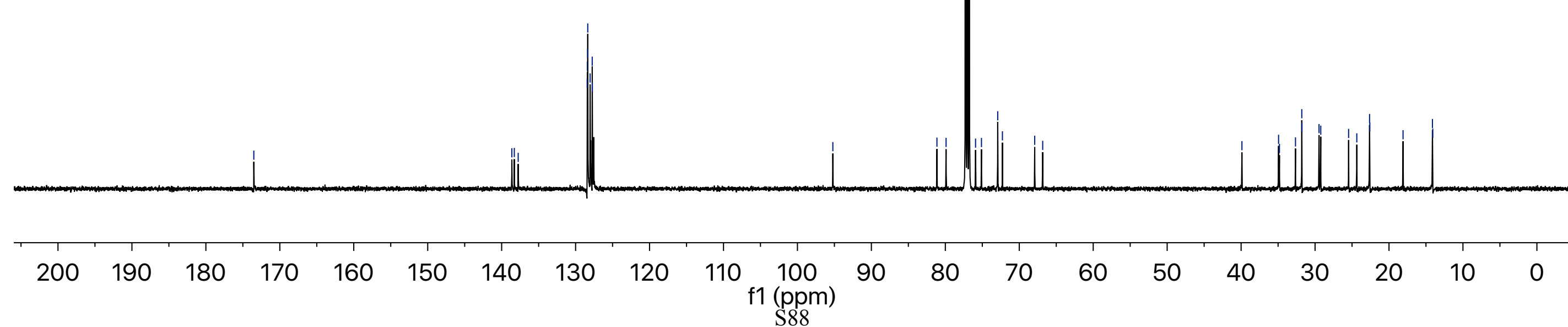


28-H - WL-1-096-product — 599.926 MHz H1 1D in cdcl3 (ref. to CDCl3 @ 7.26 ppm), temp 25.8 C -> actual temp = 27.0 C, autoxid probe —

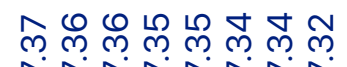

<smiles>CCCCCCCC(=O)N1C(=O)OC[C@H]1Cc1ccccc1</smiles>

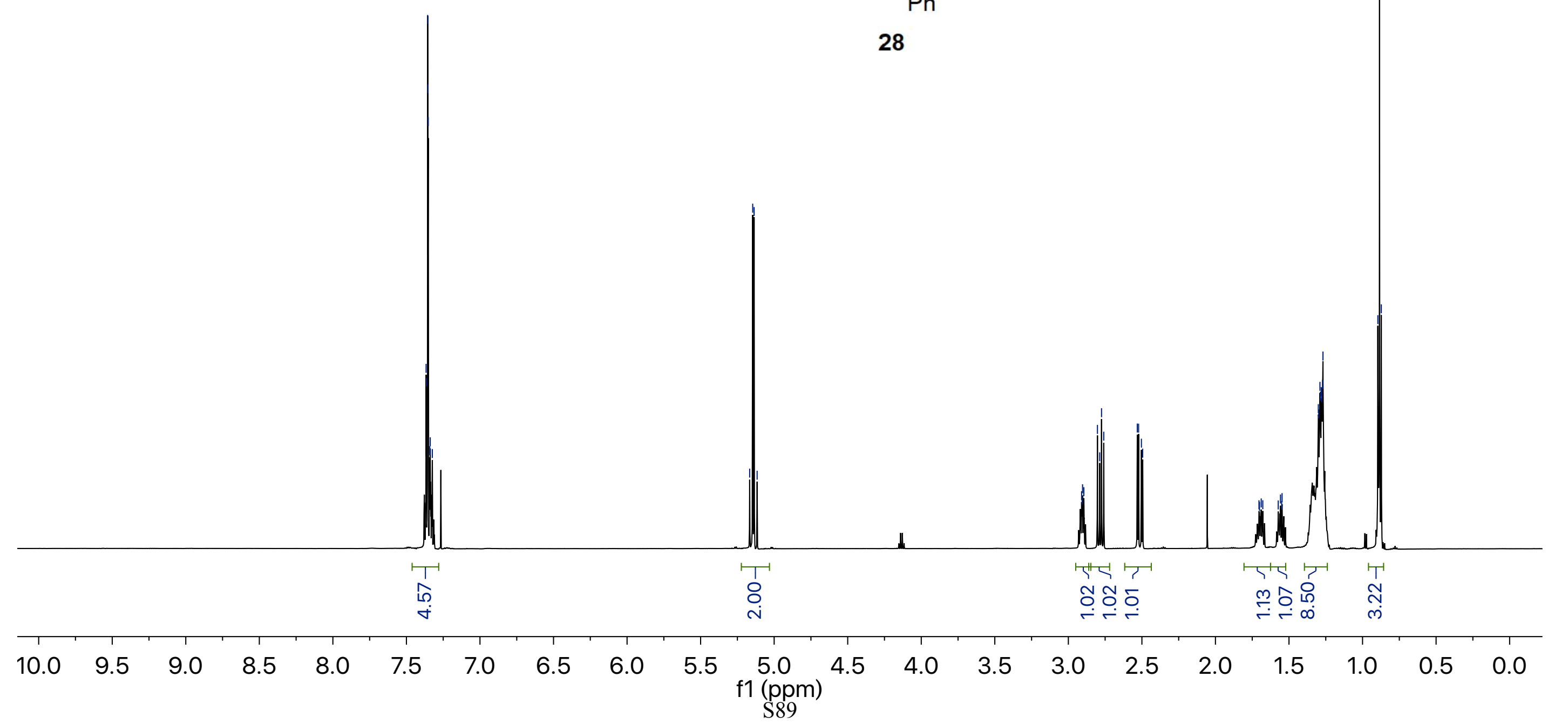


28-C - WL-1-096-CNMR - 150.868 MHz C13[H1] 1D in cdcl3 (ref. to CDCl3 @ 77.06 ppm), temp 25.8 C -> actual temp = 27.0 C, autoxid probe -
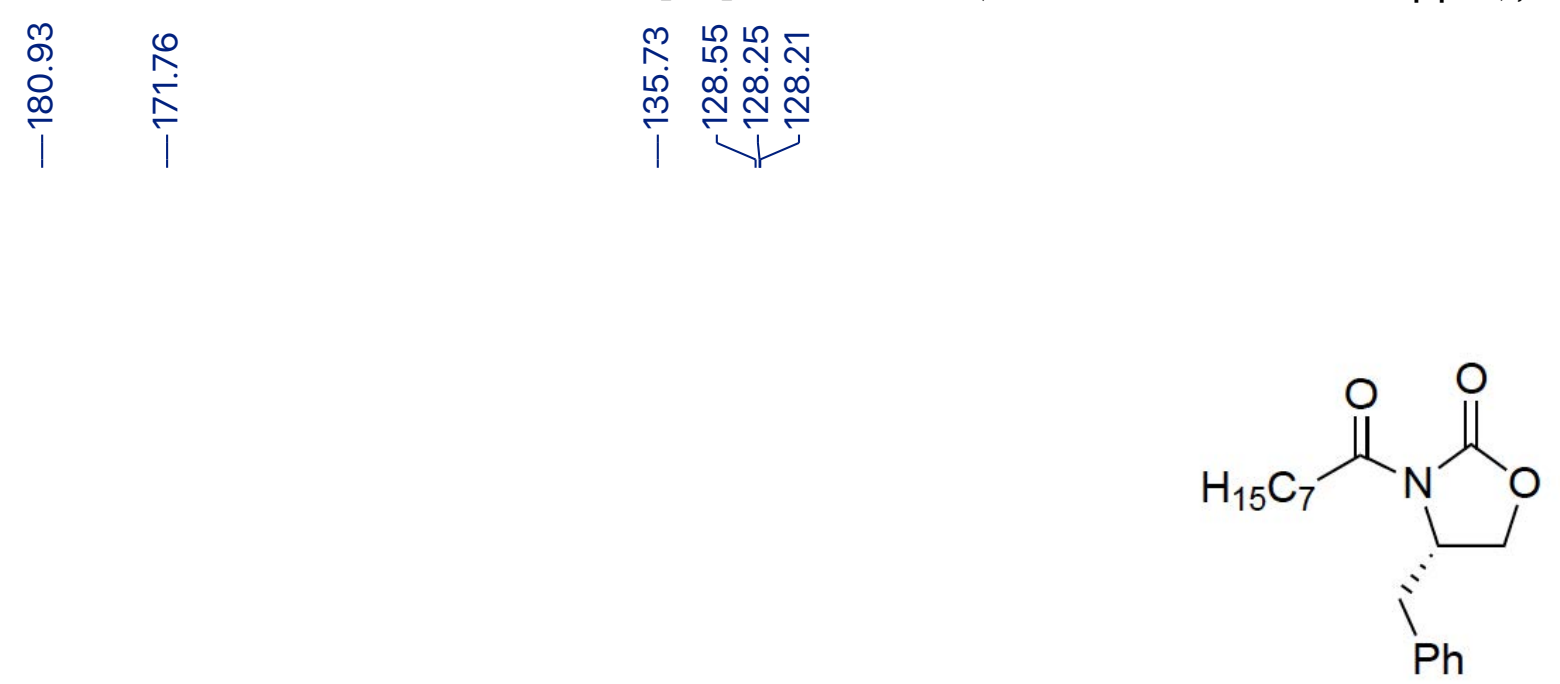

28

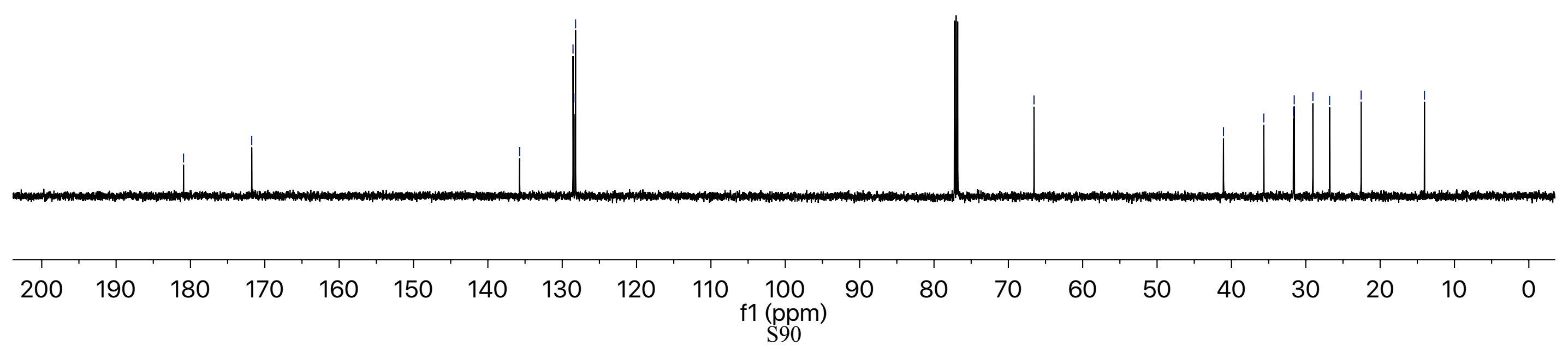


<smiles>CCCCC(CC(=O)OCc1ccccc1)C(=O)N1C(=O)OC[C@H]1CPc1ccccc1</smiles>

29

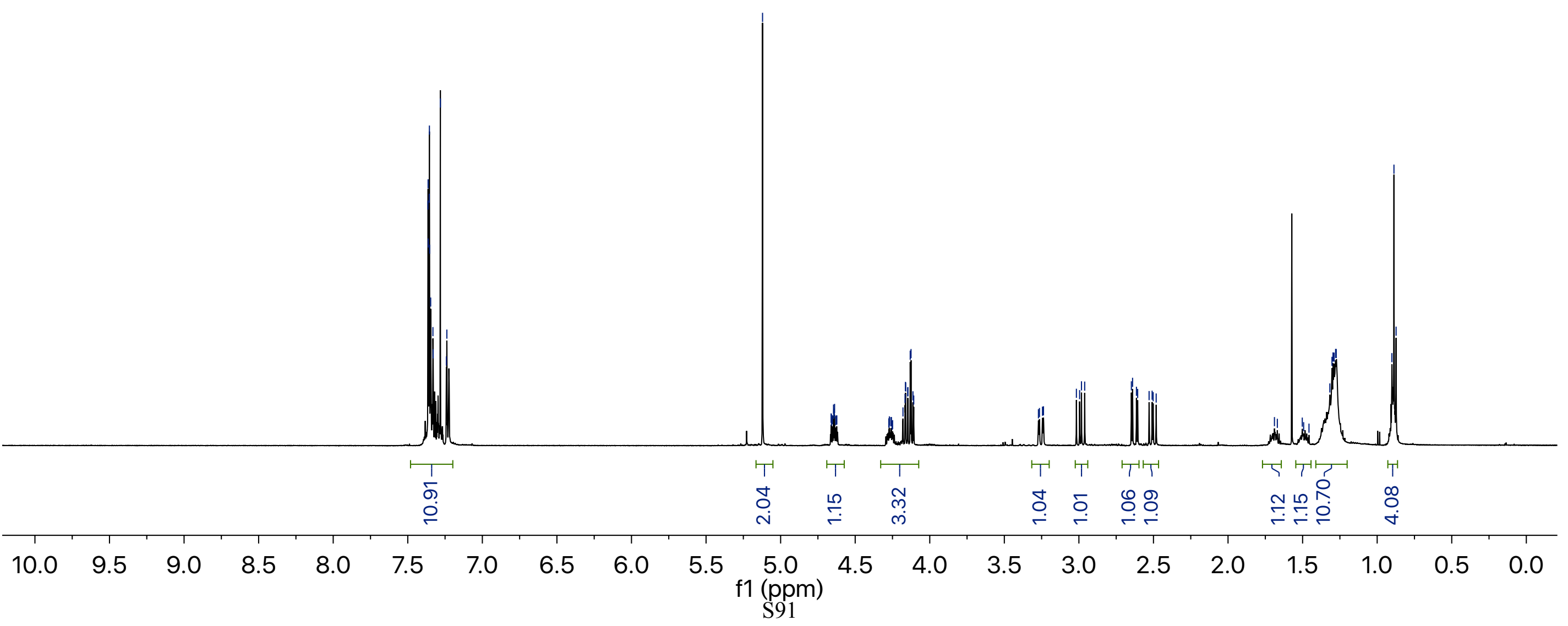


29-C - WL-2-036-product-CNMR - 125.266 MHz C13[H1] 1D in cdcl3 (ref. to CDCl3 @ 77.06 ppm), temp 27.2 C -> actual temp = 27.0 C, autoxc

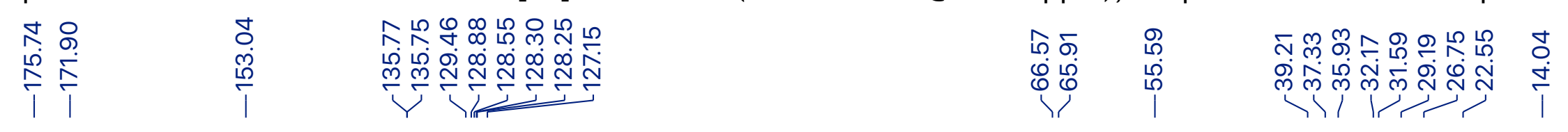<smiles>O=C(O)C[C@@H](C(=O)Oc1ccccc1)C(=O)N1C(=O)OC[C@H]1Cc1ccccc1</smiles>

29

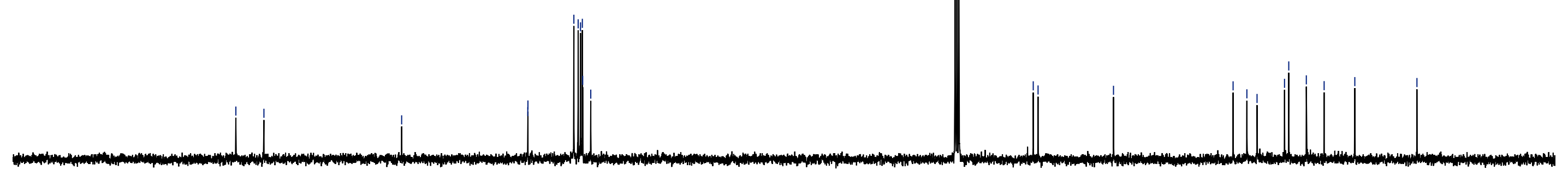

$\begin{array}{llllllllllllllllllllll}200 & 190 & 180 & 170 & 160 & 150 & 140 & 130 & 120 & 110 & \begin{array}{c}100 \\ \mathrm{f} 1(\mathrm{ppm}) \\ \mathrm{S} 92\end{array} & 90 & 80 & 70 & 60 & 50 & 40 & 30 & 20 & 10 & 0\end{array}$


30-H - Lei, WL-1-087-001 - 499.815 MHz H1 PRESAT in cdcl3 (ref. to CDCl3 @ 7.26 ppm), temp 27.7 C -> actual temp = 27.0 C, colddual probe

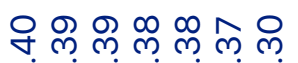

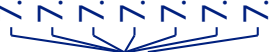

ํำำ

مان

苜心

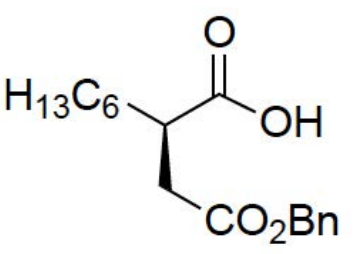

30
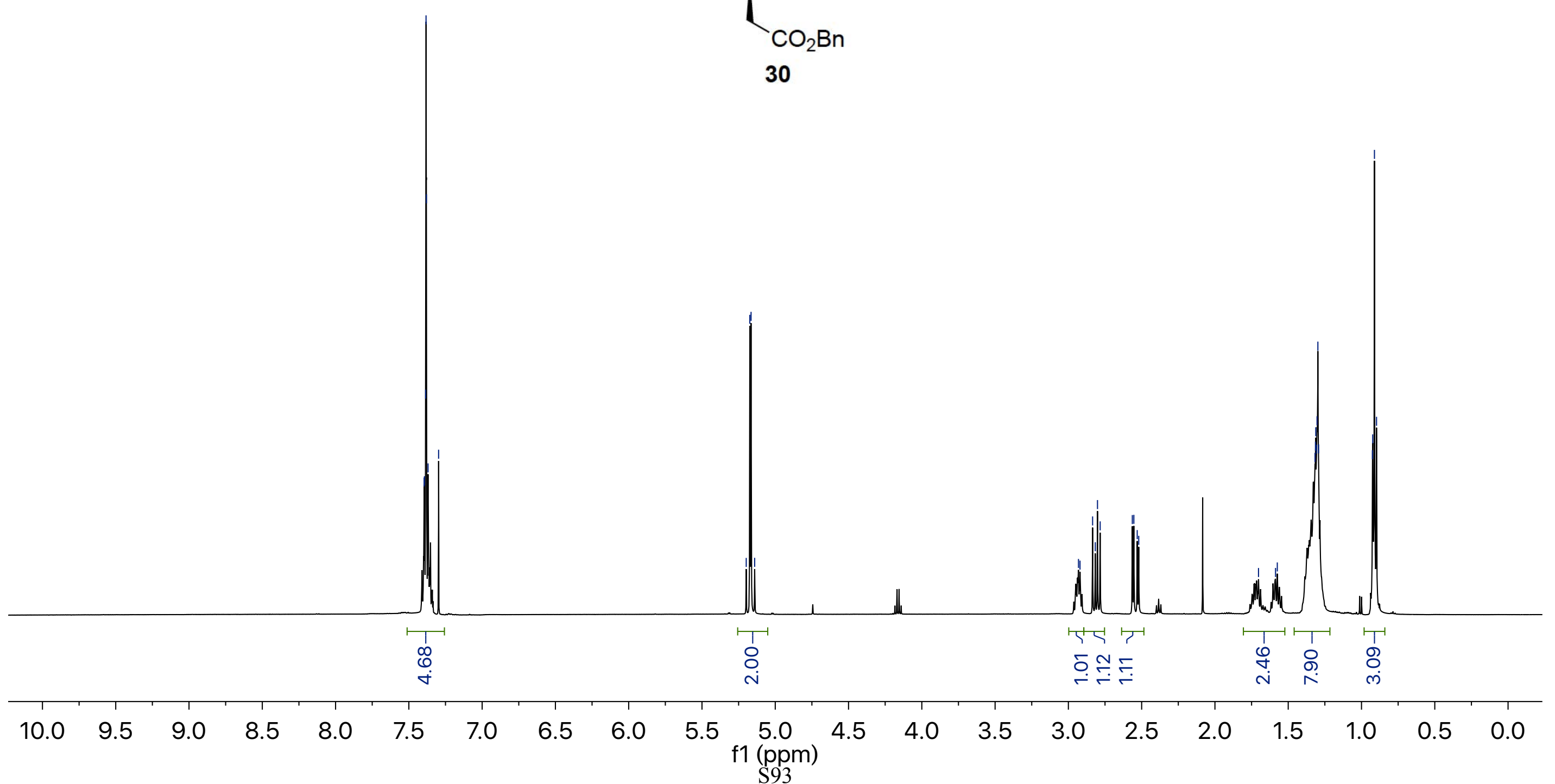
30-C - Lei, WL-1-087-001 - 125.693 MHz C13[H1] 1D in cdcl3 (ref. to CDCl3 @ 77.06 ppm), temp $27.7 \mathrm{C}->$ actual temp = 27.0 C, colddual probı

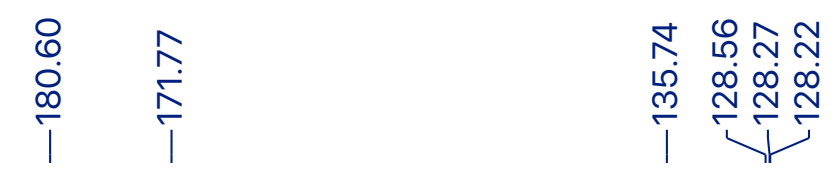

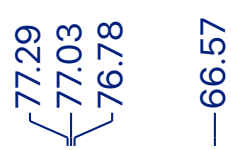

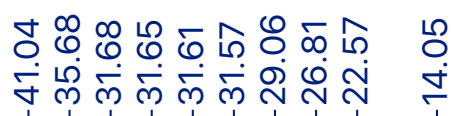<smiles>CCCC(CC(=O)O)C(=O)O</smiles>

30
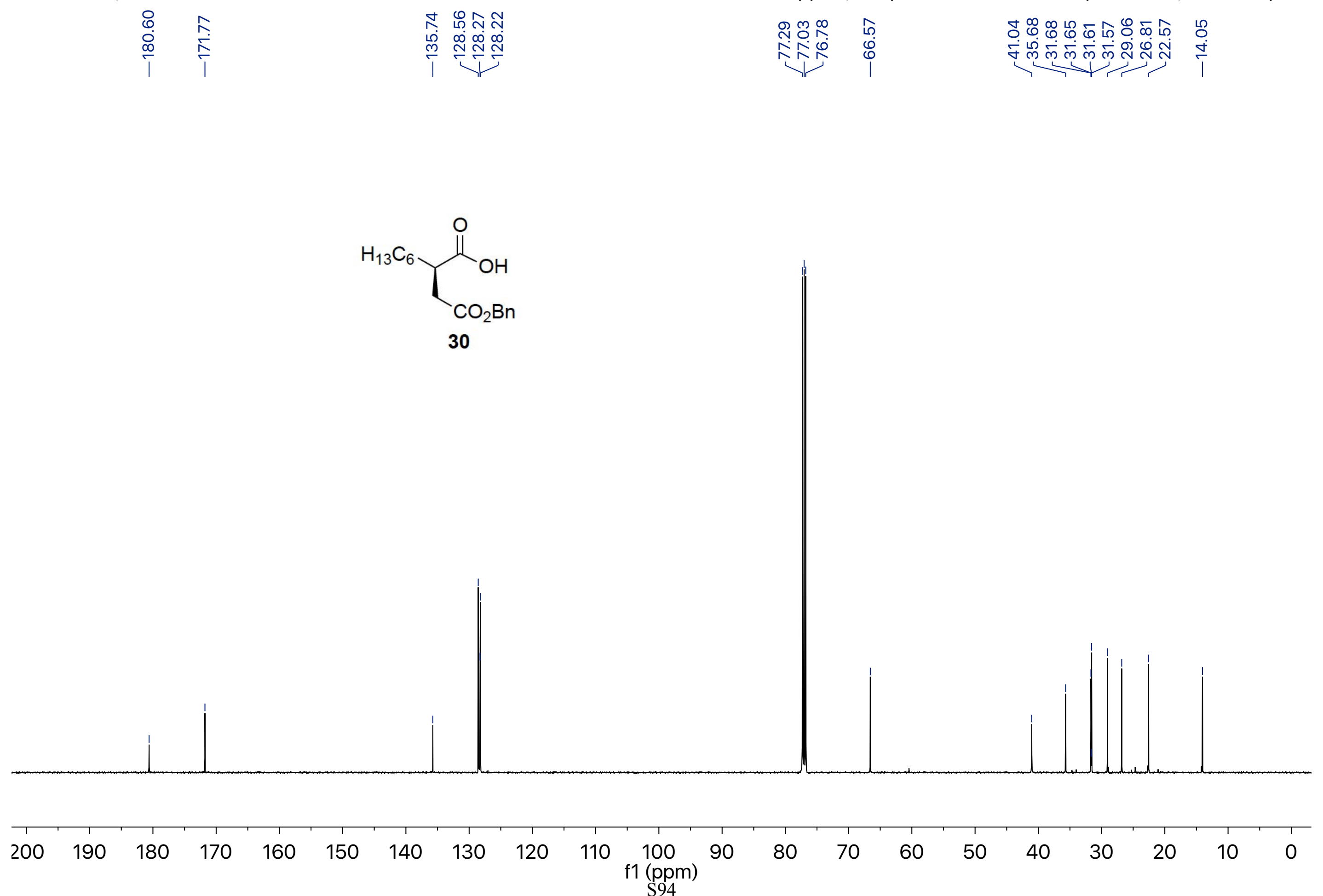
31-H - Wl-2-067-prod - 498.118 MHz H1 1D in cdcl3 (ref. to CDCl3 @ 7.26 ppm), temp 27.2 C $->$ actual temp = 27.0 C, autoxdb probe -



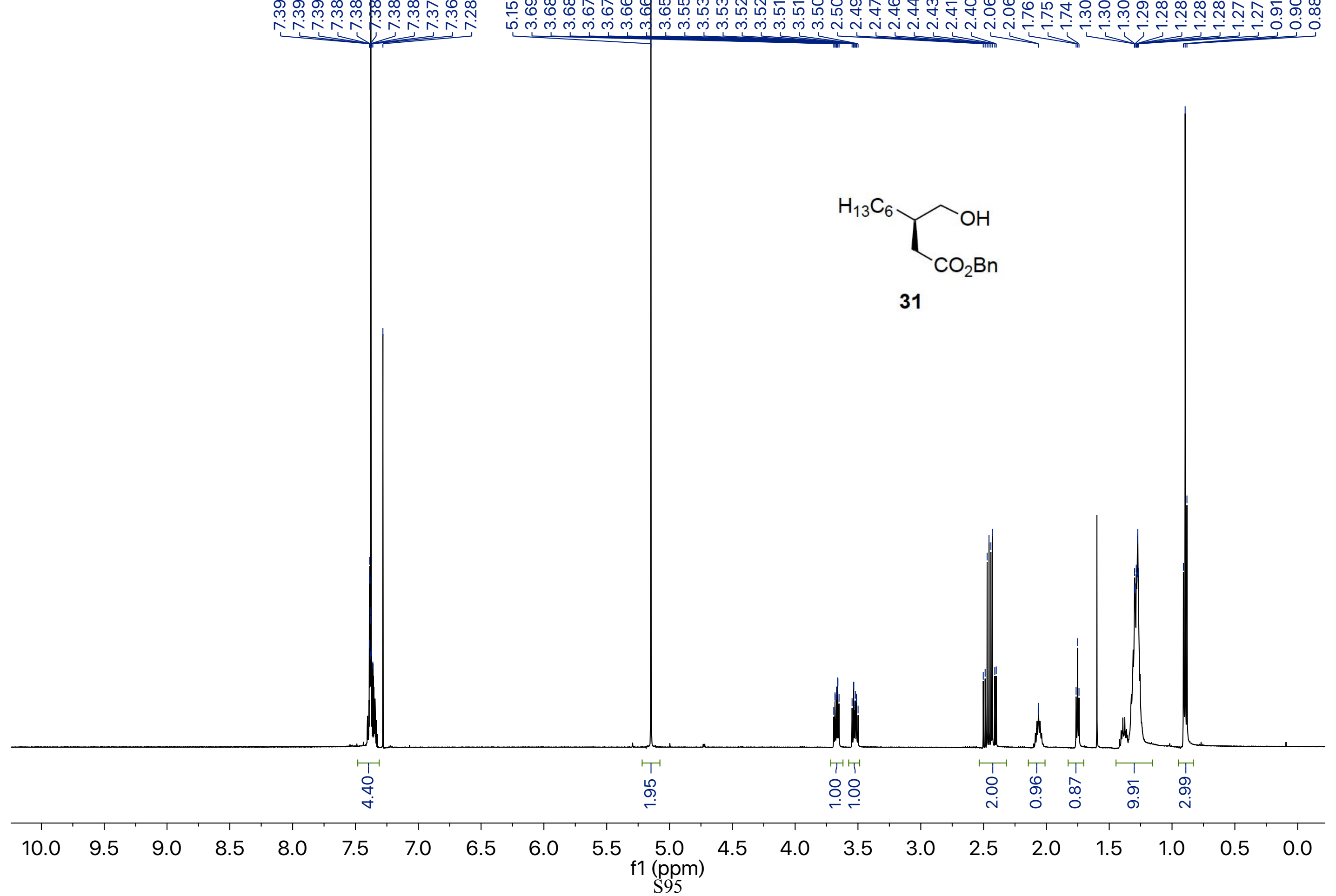


31-C - WI-2-067-prod-CNMR - 125.266 MHz C13[H1] 1D in cdcl3 (ref. to CDCl3 @ 77.06 ppm), temp 27.2 C -> actual temp = 27.0 C, autoxdb pr



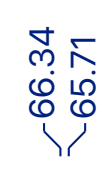

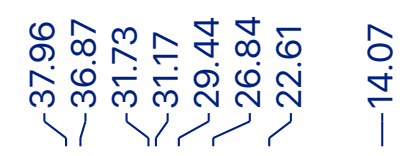

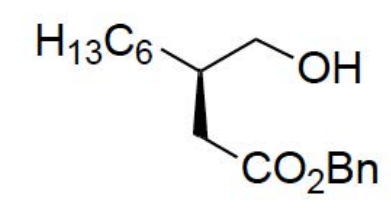

31

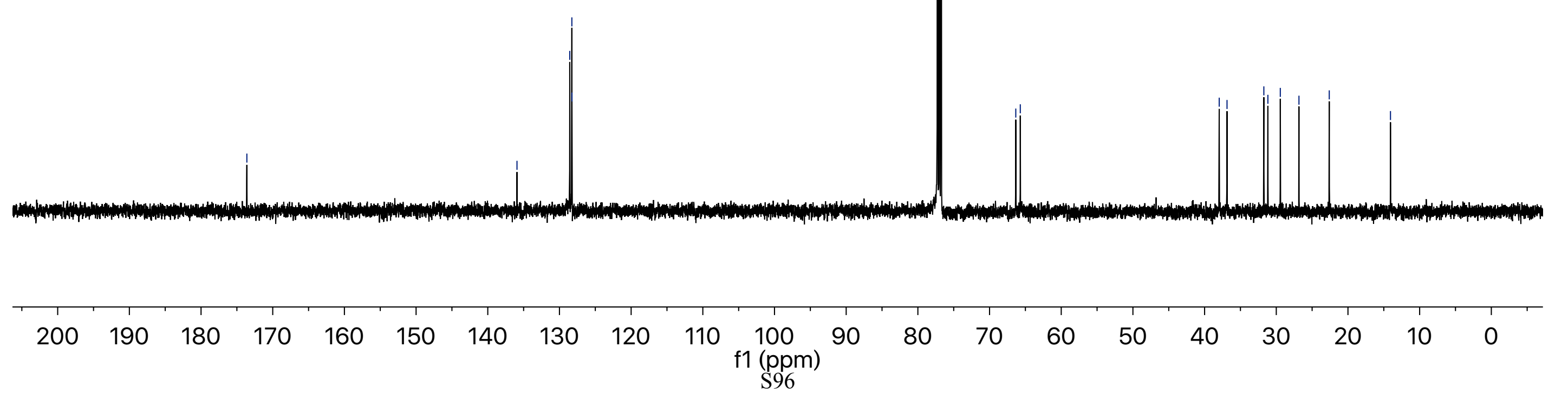


33-H - WL-1-093-002 - 599.926 MHz H1 1D in cdcl3 (ref. to CDCl3 @ 7.26 ppm), temp 25.8 C $>>$ actual temp = 27.0 C, autoxid probe -

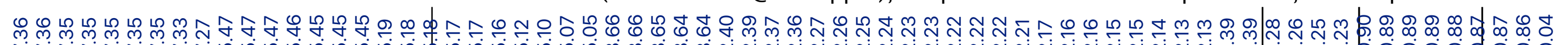

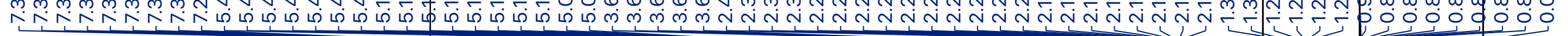

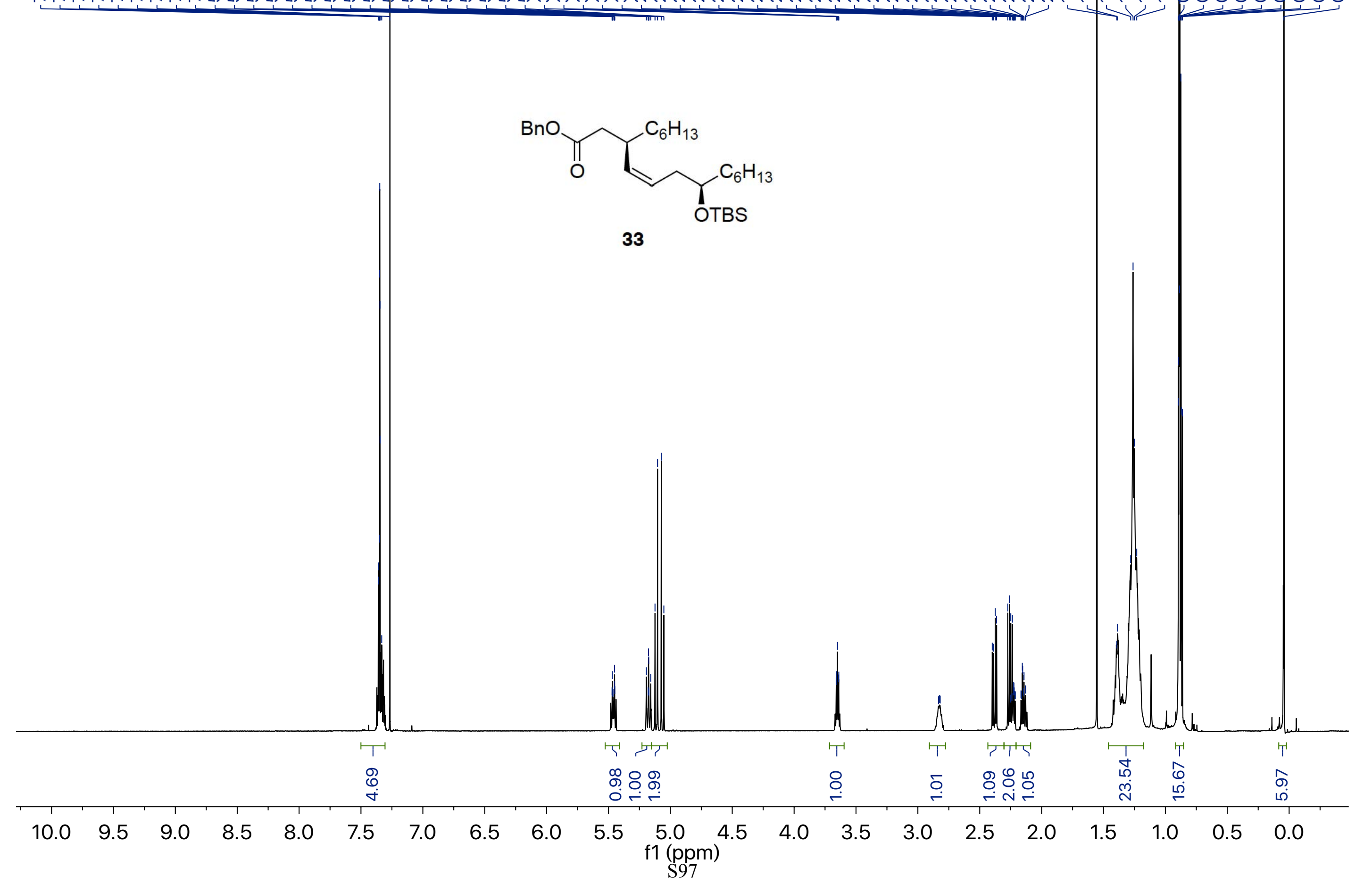


33-C - Lei, WL-1-073-CNMR - 125.693 MHz C13[H1] 1D in cdcl3 (ref. to CDCl3 @ 77.06 ppm), temp $27.7 \mathrm{C}->$ actual temp = 27.0 C, colddual pr 采

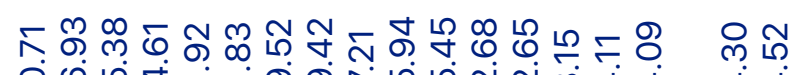

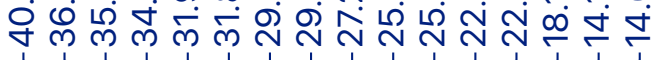<smiles>CCC(C/C=C\C(CC(=O)O[GaH2])c1ccccc1)c1ccccc1</smiles>

33
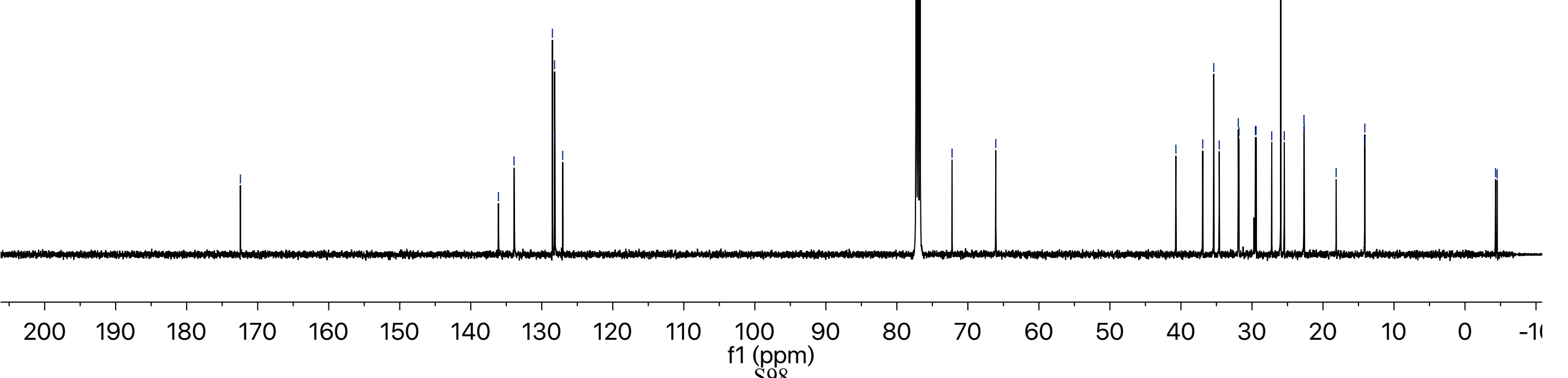
S98 
34-H - WL-2-058-prod-a - 399.794 MHz H1 1D in cdcl3 (ref. to CDCl3 @ 7.26 ppm), temp 27.0 C -> actual temp = 27.0 C, autoxdb probe -

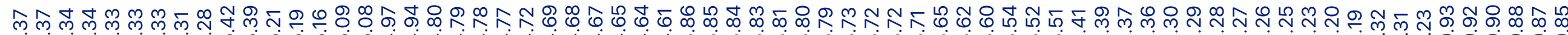


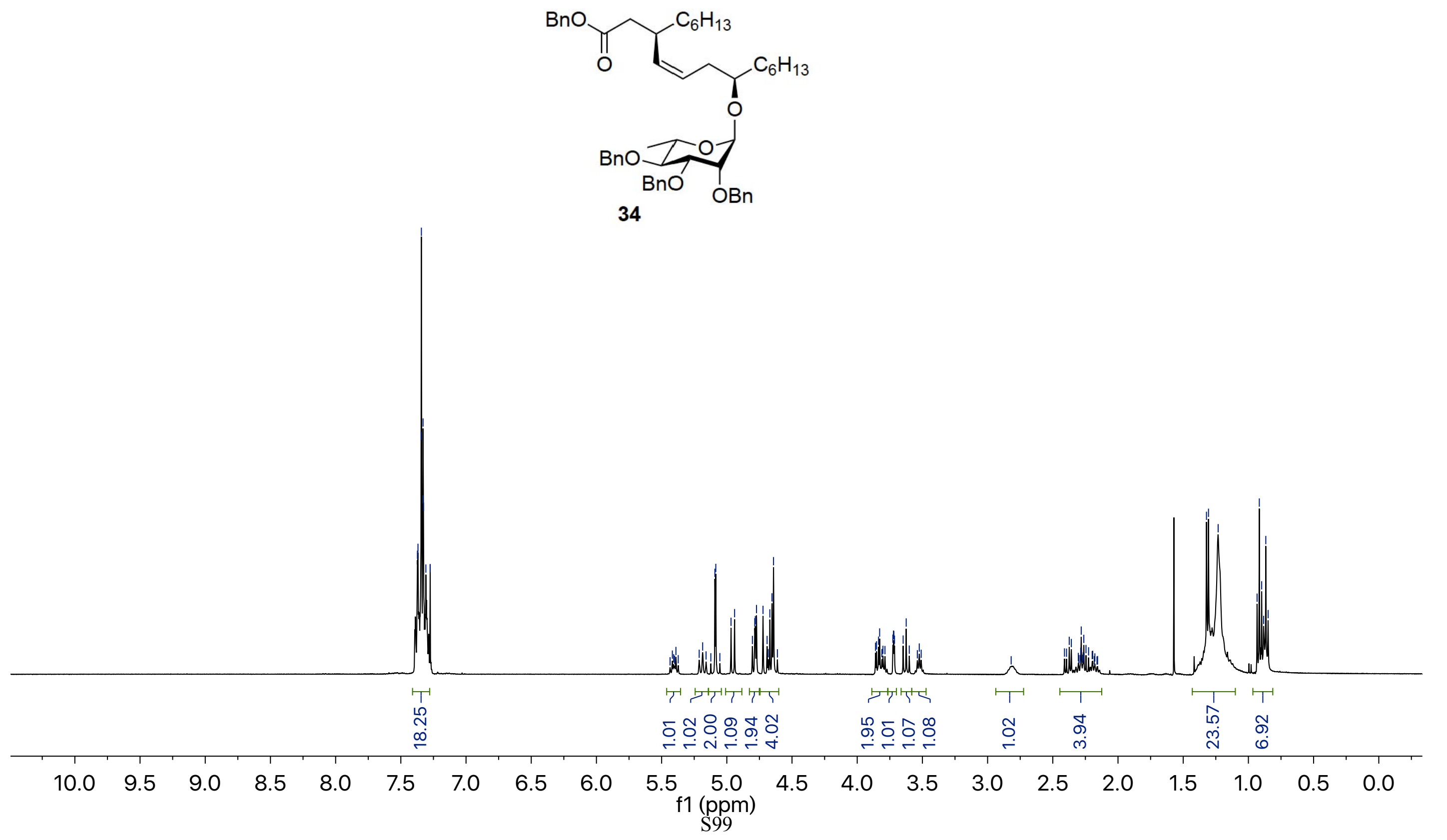
34-C - Lei, WL-2-025-prod-a-CNMR - 125.693 MHz C13[H1] 1D in cdcl3 (ref. to CDCl3 @ 77.06 ppm), temp 27.7 C -> actual temp = 27.0 C, coll

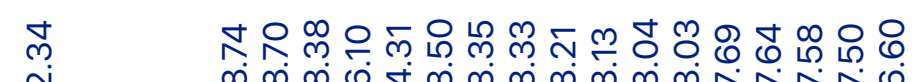

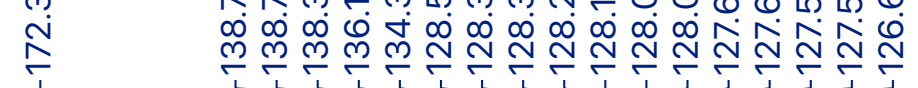

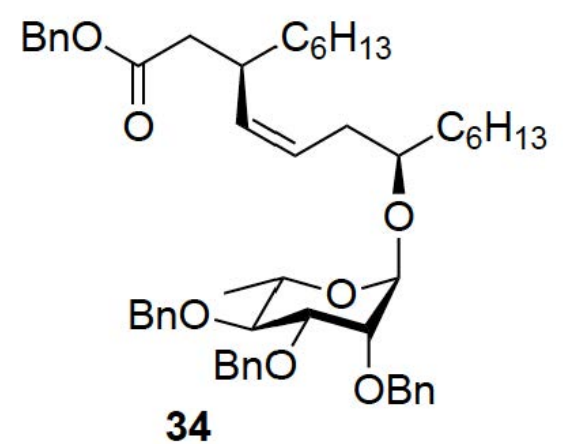

34
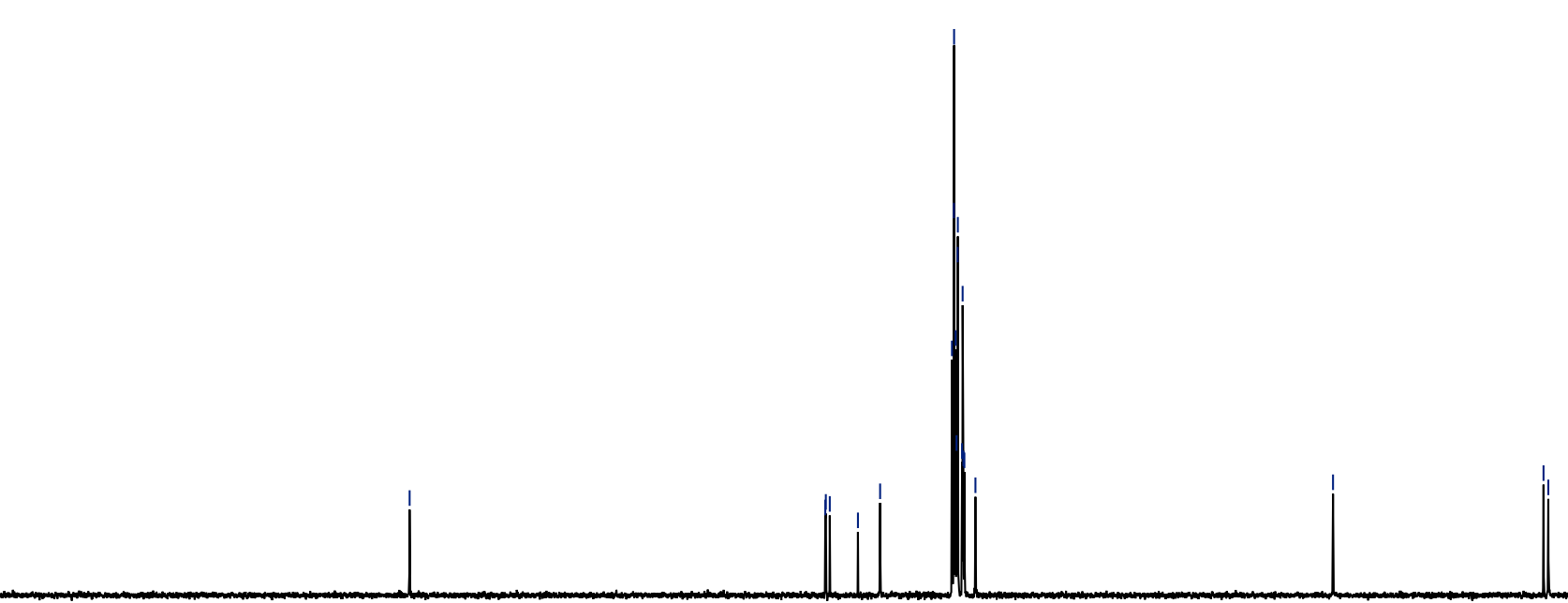

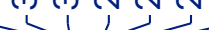

$\begin{array}{llllllllll}200 & 190 & 180 & 170 & 160 & 150 & 140 & 130 & 120 & 110 \\ \mathrm{f} 1(\mathrm{ppm})\end{array}$
S100 


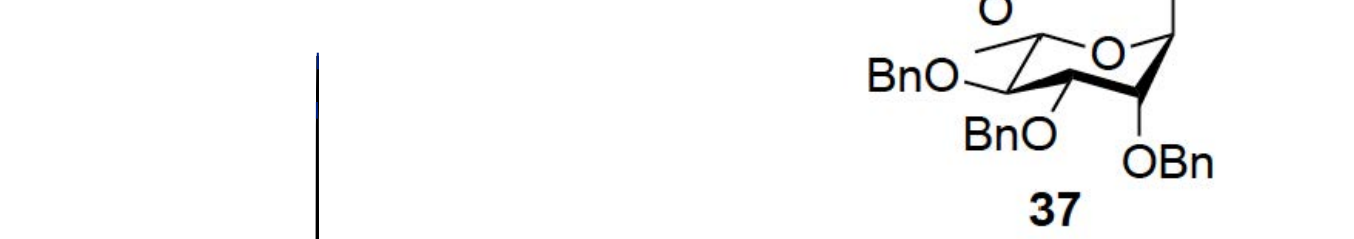

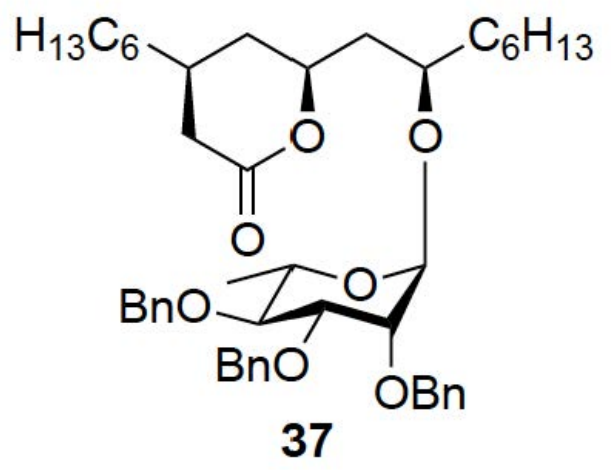<smiles>[Te]</smiles>
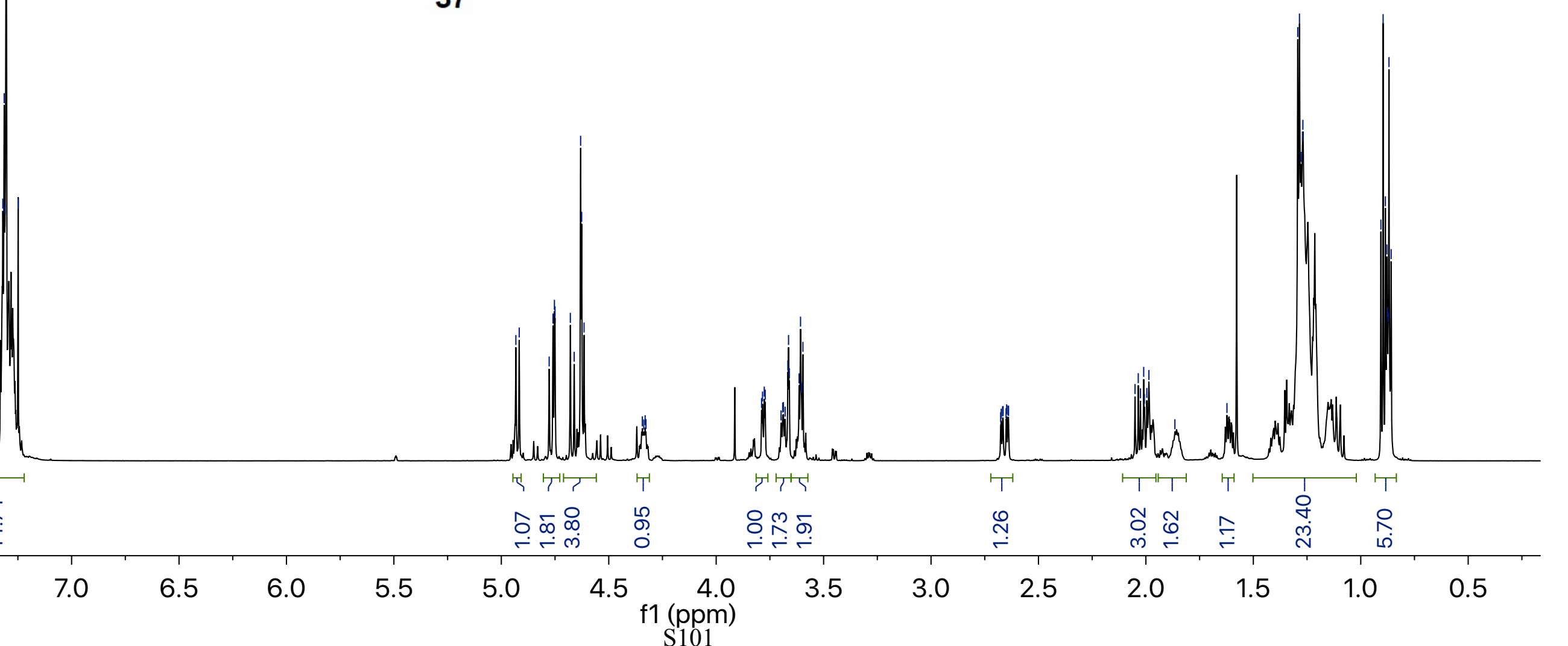
37-C - Lei, WL-2-076-prod - 125.691 MHz C13[H1] 1D in cdcl3 (ref. to CDCl3 @ 77.06 ppm), temp 27.7 C -> actual temp = 27.0 C, colddual prob

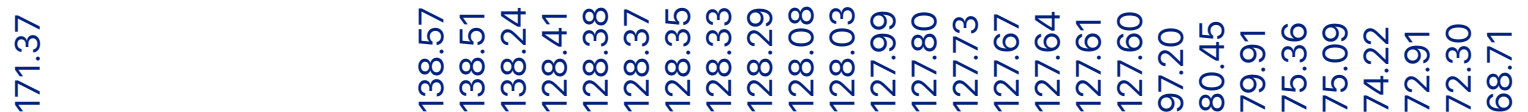
$\stackrel{\stackrel{1}{*}}{1}$

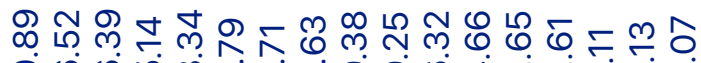
ச



37



\begin{tabular}{|c|c|c|c|c|c|c|c|c|c|c|c|c|c|c|c|c|c|c|c|c|}
\hline 200 & 190 & 180 & 170 & 160 & 150 & 140 & 130 & 120 & 110 & $\begin{array}{c}100 \\
\text { f1 (ppm) } \\
\text { S102 }\end{array}$ & 90 & 80 & 70 & 60 & 50 & 40 & 30 & 20 & 10 & 0 \\
\hline
\end{tabular}




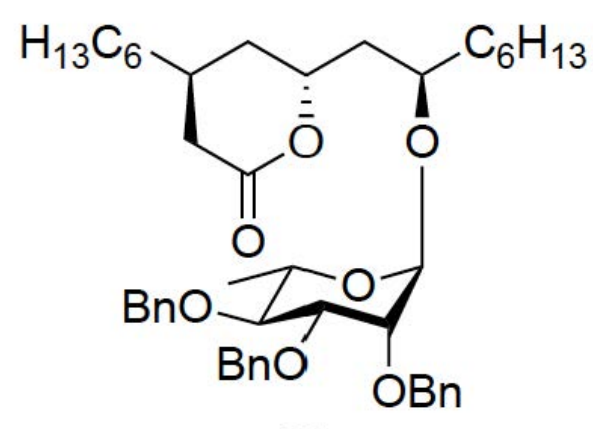

38

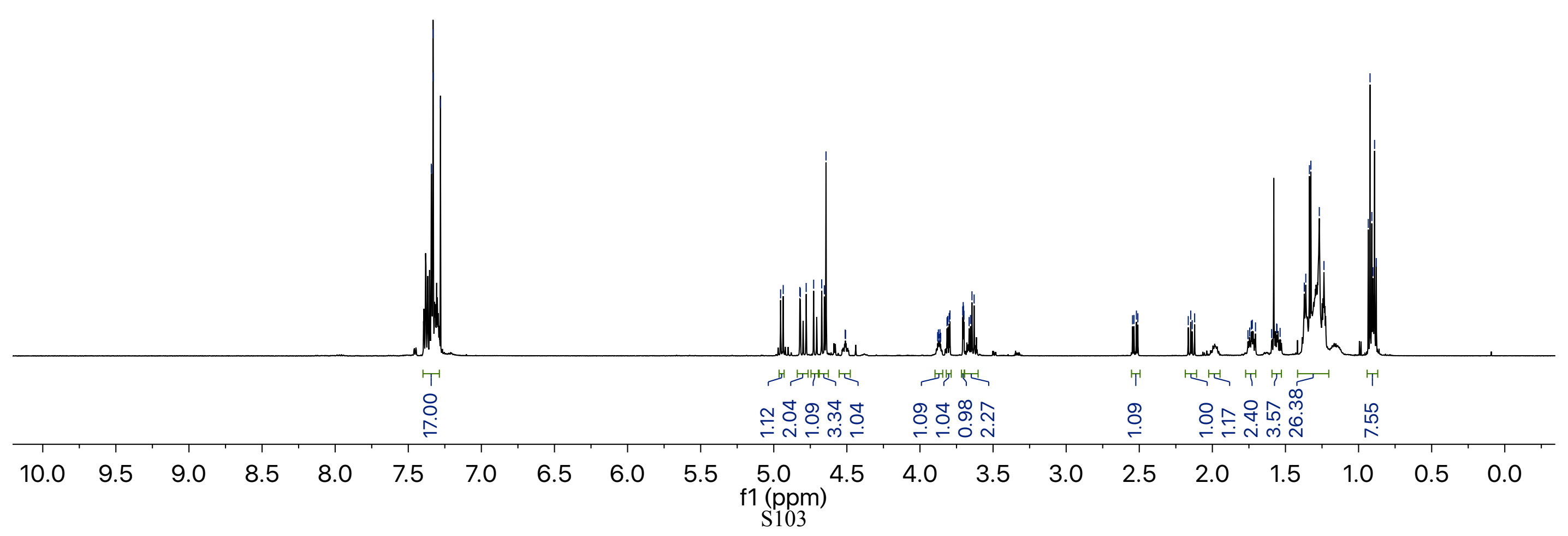



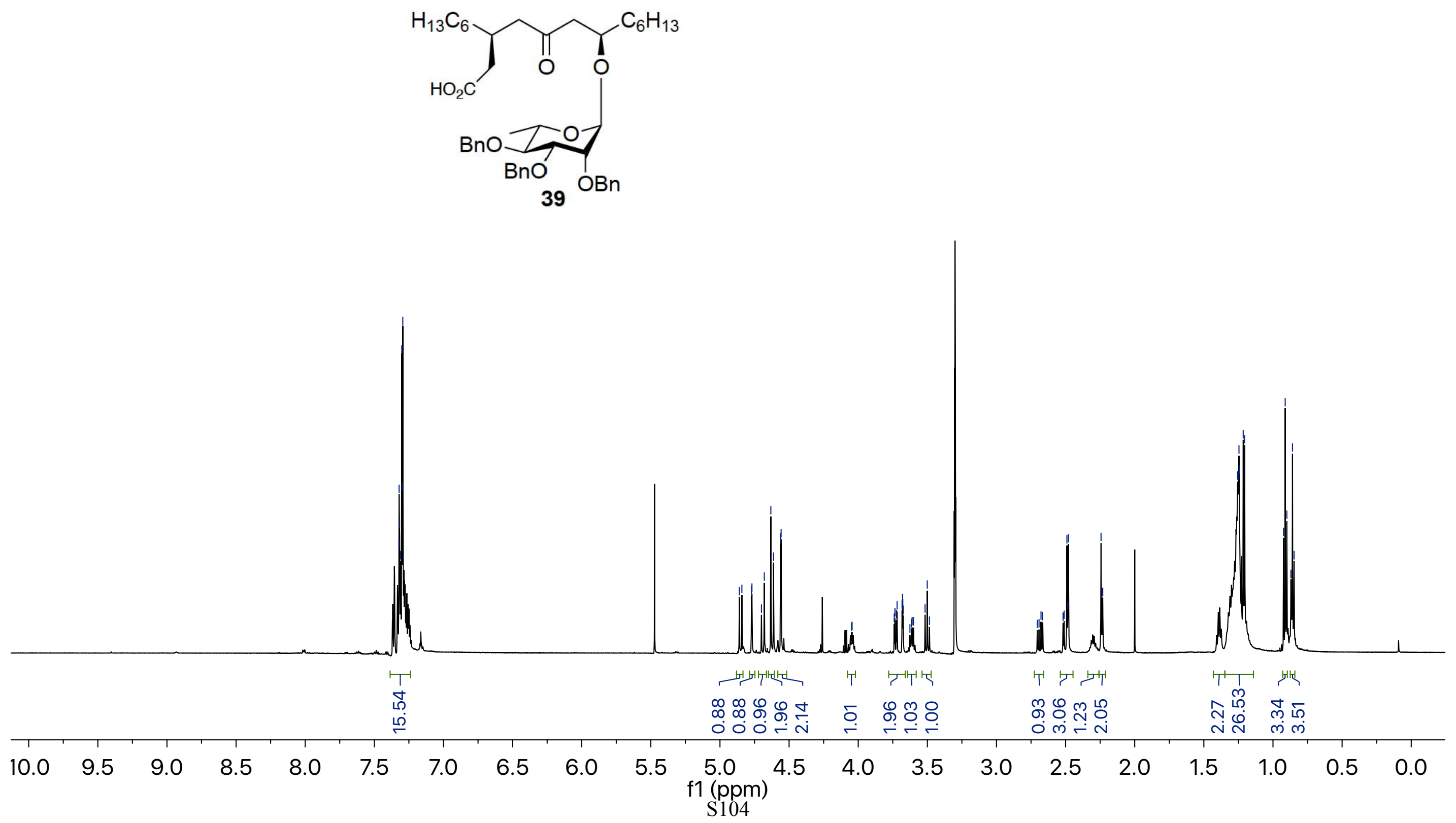
